Portland State University

PDXScholar

Spring 6-17-2014

\title{
Trauma-Informed Research and Planning: Understanding Government and Urban Native Community Partnerships to Addressing Substance- Exposed Pregnancies in Portland, OR
}

Amanda Mercier

Portland State University

Follow this and additional works at: https://pdxscholar.library.pdx.edu/open_access_etds

Part of the Indigenous Studies Commons, Substance Abuse and Addiction Commons, and the Women's Health Commons

Let us know how access to this document benefits you.

\section{Recommended Citation}

Mercier, Amanda, "Trauma-Informed Research and Planning: Understanding Government and Urban Native Community Partnerships to Addressing Substance-Exposed Pregnancies in Portland, OR" (2014). Dissertations and Theses. Paper 1803.

https://doi.org/10.15760/etd.1803

This Thesis is brought to you for free and open access. It has been accepted for inclusion in Dissertations and Theses by an authorized administrator of PDXScholar. Please contact us if we can make this document more accessible: pdxscholar@pdx.edu. 
Trauma-Informed Research and Planning: Understanding Government and Urban Native Community Partnerships to Addressing Substance-Exposed Pregnancies in Portland, OR

by

Amanda Mercier

A thesis submitted in partial fulfillment of the requirements for the degree of

\author{
Master of Science \\ in \\ Sociology \\ Thesis Committee: \\ Maura Kelly, Chair \\ Matthew Carlson \\ Kelly Gonzales \\ José Padín
}

Portland State University

2014 
(C) 2014 Amanda Mercier 


\begin{abstract}
In 2011, representatives from the Multnomah County Health Departments and several Native-serving organizations came together to address substance-exposed pregnancies among urban Native Americans in Portland, Oregon. From these partnerships, the Future Generations Collaborative was formed representing a significant shift toward community-led maternal child health research and planning. Additionally, the Future Generations Collaborative adopted a historical trauma-informed community based participatory research and planning process. This is particularly significant considering government agencies' role in colonization within Native communities. The purpose of this case study is to explore partnerships between government agencies and the Portland Native community within the Future Generations Collaborative. Given the profound influence of historical trauma in Native communities, this paper addresses how the partnerships between government agencies and the Portland Native community pose distinct opportunities, challenges, and implications.

Drawing from FGC members' lived experiences and an interdisciplinary body of research, I develop a theoretical model for explaining the government's role in creating and sustaining historical trauma within Native communities. This analysis provides critical context for examining the impact of historical trauma on the relationships between government agencies and the Portland Native community within the FGC. By entering methodological discussions of Native-specific community-based participatory research, this study also addresses how the use of a trauma-informed research and planning model affects the relationships between government agencies and the Portland Native community within the FGC.
\end{abstract}




\section{Dedication}

This thesis is dedicated to my loving husband, Jordan Mercier who has shared in my struggles and achievements throughout this research process. Thank you for being my inspiration, my role model, and my solace. I also dedicate this thesis to all of the sweet babies of the Future Generation Collaborative - especially, our own daughter, Ila Mercier. I hope that this work can contribute to their continued health and happiness in some small way. 


\section{Acknowledgements}

First and foremost, I would like to acknowledge all the members of the Future Generations Collaborative (FGC) for making this work possible and for guiding this research a hundred percent of the way. These are your stories and therefore this work belongs to you. I only hope that this thesis can be useful to the collaborative and that it truly honors the voices of FGC members. All the members of the FGC deserve recognition for their outstanding accomplishments and their role within crafting this thesis. I have learned so much from all of you. This work has also been influenced profoundly by the wisdom and strength of the Elders and Natural Helpers. Thank you for being such positive role models to me. I want to thank Charmaine Kinney, Donita Fry, Jillene Joseph, and Heather Heater for going above and beyond as mentors to me and for taking time out of their busy schedules to share their invaluable expertise. I also want to thank the FGC for the impact they have had on my life, personally and professionally. Being a member of the FGC has been a transformational experience and I look forward to many more years of collaboration.

I would like to acknowledge my thesis chair, Dr. Maura Kelly, whose guidance, support, and flexibility throughout this research process have been immeasurable. A special acknowledgement also belongs to Dr. Kelly Gonzales who provoked a shift within my own research, brought me into the FGC, and who mentored me throughout this work. Thank you also to Dr. Matt Carlson and Dr. José Padín for supporting me on my committee and offering your insight into this work. I am truly appreciative of Greg Archuleta, Cheryle Kennedy, Dr. Cornel Pewewardy, Dr. June Strickland, and Dr. 
Matthew Town, who have all supported me, offering me resources and guidance as I began my work in the Portland Native community.

To all my family who supported me during my Master's program, I thank you from the bottom of my heart. I want to especially thank my mom and dad, Jeanette and Roger who have always championed my academic pursuits and freely shared their love, pride, and encouragement. Great thanks also belong to my parents-in-law, Tammy and Mike, for listening to me ramble on about my thesis and showing up at all hours of the night bearing food. Finally, I want to acknowledge my husband, Jordan Mercier for all the around-the-clock research advice he has provided me and for the profound influence he has on this work. 


\section{Table of Contents}

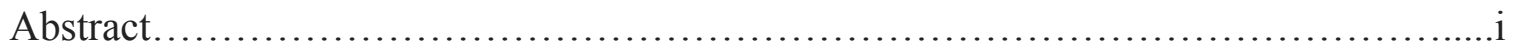

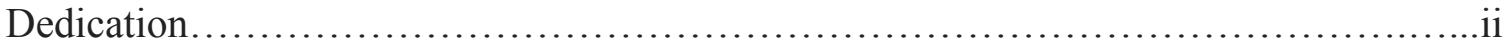

Acknowledgements...............................................................

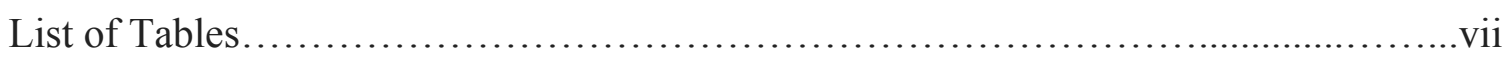

List of Figures..............................................................

Chapter 1

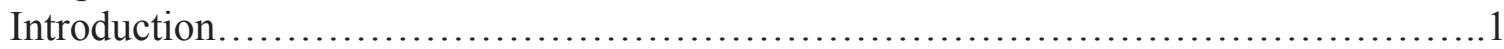

Chapter 2

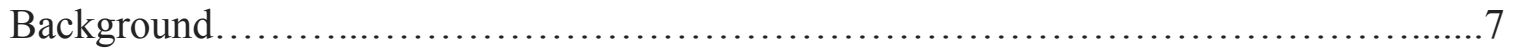

Chapter 3

Literature Review........................................................... 16

Chapter 4

Methods..................................................................... 40

Chapter 5

Findings on the Historical Trauma Process........................................57

Chapter 6

Findings on the FGC's "Shift" to a Trauma-Informed Model.

Chapter 7

Findings on the Success of FGC's Trauma-Informed Model........................105

Chapter 8

Findings on the Barriers to the FGC's Trauma-Informed Model.....................142

Chapter 9

Discussion................................................................ 160

References..................................................................... 175

Appendices 


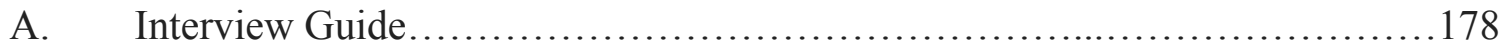

B. Codebook Revisions and Themes......................................... 180 


\section{List of Tables}

Table 1: Description of Interview Sample..................................... 43

Table 2: Codebook Revisions and Themes........................................ 179 


\section{List of Figures}

Figure 1: Walters and Simoni’s (2002) Indigenist Stress Coping Model................22

Figure 2: Myrdal's (1962) Vicious Cycle.....................................30

Figure 3: The Historical Trauma Process.......................................... 32

Figure 4: Findings on the Historical Trauma Process............................90

Figure 5: Findings on the FGC's Trauma-Informed Process......................... 105 


\section{Chapter 1: Introduction}

Beginning in 2011, representatives from Multnomah County Health Department and several Native American serving organizations ${ }^{2}$ came together to address substanceexposed pregnancies among Native Americans ${ }^{2}$ in Portland, OR. These partnerships resulted in the formation of the Native maternal child health collaborative, the Future Generations Collaborative (FGC). The FGC adopted a community-based participatory research and planning (CBPRP) framework for conducting Native-driven research and creating health promotion strategies for the prevention of substance-exposed pregnancies. The research presented draws on ethnographic data collected at FGC meetings and events and interviews with FGC members. By examining FGC member's evaluations of historical trauma and the FGC's trauma-informed research and planning model, this case study explores the collaborative partnerships between government agencies and the Portland Native community within the context of FGC.

The process used by the FGC has many similarities to other community-based participatory research (CBPR) projects. CBPR is a widely used model for research and practice within Native American populations (Holkup et al. 2009). One defining feature of CBPR is to include members of the study population within each phase of the research process as equal partners (Holkup et al. 2009). However, the FGC's model is distinct considering the fact that public health initiatives for the prevention of substance-exposed

\footnotetext{
${ }^{2}$ Native American serving organizations include Native Wellness, Northwest Portland Area Indian Health Board (NPAIHB), Native American Rehabilitative Association of the Northwest (NARA), Native American Youth and Family Center (NAYA), Healing Feathers, and the Portland State Native American Student and Community Center (NASCC).

${ }^{2}$ Throughout this paper I use the terms "Native American" and "Native" to describe the population of indigenous North American peoples, also known as American Indian/Alaskan Natives. This research acknowledges the political significance and contested views of how Native Americans have been named in research and seeks to use terms that are in line with the members of the population being studied.
} 
pregnancies have rarely been specifically designed to serve Native American populations (Mehl-Madrona 2000). Such initiatives have often proved inadequate due to a lack of culturally appropriate design and implementation (Mehl-Madrona 2000). Moreover, culturally appropriate public health initiatives specific to the urban Native community in Portland, Oregon are also lacking. This is problematic considering Portland, OR represents the ninth largest urban Native American population in the United States and is a site where many health and social disparities are more severe for the local Native community than in other parts of the country (Curry-Stevens et al. 2011). According to a 2010 community-validated population count, the Native American population of Multnomah County totals at 40,783 (Curry-Stevens et al. 2011).

The partnerships within the FGC between local, state, non-government organizations $^{3}$, and community stakeholders represent a significant and intentional shift toward community-led Native American maternal child health planning in Portland, OR. According to the FGC, this Native-driven approach creates the opportunity for integration of Native American knowledge, beliefs, and practices into the research and planning process. Finally, the FGC research design is guided by theory of historical trauma. Historical trauma in Native communities acknowledges the intergenerational transmission of collective and cumulative trauma from colonization and the persistent effects on Native Americans today (Evans-Campbell 2008). The use of historical trauma as a guiding theory represents another major shift for collaborations involving the

\footnotetext{
3 The Oregon Health Authority and Planned Parenthood represent two additional partnering organizations within the Future Generations Collaborative that are neither explicitly Native-serving nor governmental.
} 
governmental agencies, especially considering the role the U.S. government has had in creating historical trauma within Native communities.

\section{Research Questions}

The purpose of this case study is to explore the collaborative partnerships between government agencies and the Portland Native community within the FGC. To this end, FGC member evaluations will be used to access the affects of historical trauma and the FGC's trauma-informed model on the these collaborative partnerships. FGC members' evaluations of historical trauma and CBPR will be generally defined as phenomenological reflections on lived experiences and a process of knowledge creation based on experience that informs future action (Springett and Wallerstein 2008). In this way, FGC members' evaluations are essential to addressing this studies research questions. The three central research questions of this case study are:

1. How do FGC Members describe the government's role in creating and sustaining historical trauma within Native communities?

2. How does historical trauma affect the relationships between government agencies and the Portland Native community within the FGC?

3. How does the FGC's trauma-informed community-based participatory research and planning process affect the relationships between government agencies and the Portland Native community within the FGC?

The first two research questions speak to the broader themes of what it means for Native communities to come into partnership with the colonizer, governmental agencies, given legacy of historical trauma from colonization. The third research question addresses whether or not it is possible for Native communities and agencies of the colonizer to come together to address problems created by colonization, thereby creating a decolonizing partnership between government agencies and Native communities. The 
third research question also speaks to the degree to which indigenous resistance can be successful within the context of partnerships between agencies of the colonizer and Native communities.

Research questions used to access collaborative partnerships within the FGC are drawn from self-identified goals of the FGC. Furthermore, the research questions are supported from the body of research on historical trauma (Brave Heart 1998; EvansCampbell 2008; Fast and Collin-Vézina 2010; Walters and Simoni 2002) and CBPR within Native communities (Brayboy and Deyhle 2000; Holkup et al. 2004; Strickland 2006; Thomas et al. 2011; Wallerstein and Duran 2006; Wallerstein and Duran 2008; Walters et al. 2009) as well as Tribal Critical Race Theory (Beck 2002; McKinley and Brayboy 2005). As a whole, these research questions will allow for the assessment of the partnerships between government agencies and the Portland Native community within the context of the FGC, given the impacts of historical trauma and the FGC's traumainformed research and planning model.

\section{Summery of Chapters}

This thesis is divided into nine chapters. The current chapter has introduced the Future Generations Collaborative and provided the scope of this case study. In the second chapter, relevant background information is provided to explain to the relationships between government agencies and the Portland Native community within the FGC. Chapter 3 outlines an interdisciplinary body of literature, including theories related to historical trauma, tribal critical race theory, and Myrdal's (1962) vicious cycle. This theoretical framework is used to construct an analytical model for understanding the 
government's role in creating and sustaining historical trauma. Additionally, empirical studies of community-based participatory research within Native communities are also provided in this chapter. The discussion of previous literature in Chapter 3 also supports my methodological framework as presented in Chapter 4.

In Chapter 5: Findings on the Historical Trauma Process, FGC members describe the government's role in creating and sustaining historical trauma within Native communities broadly, thus addressing my first research question. Additionally, Chapter 5 contributes to the second research question by providing important context as to how historical trauma within Native communities at large shapes the relationships between government agencies and the Portland Native community within the FGC. Similarly, this chapter provides insight into the third research question by providing context for the FGC's trauma-informed model and by ultimately explaining the historical trauma that the FGC seeks to address.

Chapter 6: Findings on the FGC's Shift to a Trauma-Informed Model examines how County partner's perpetuated historical trauma through their initial approach in addressing substance-exposed pregnancies within the Portland Native community. This chapter also describes several factors that contributed to the formation of the FGC and the creation of FGC's trauma-informed model. Therefore, this chapter speaks to both the second and third research questions within this case study.

FGC members evaluate the FGC's trauma-informed model in detail in Chapters 7 and 8. First in Chapter 7: Findings on the Successes of the FGC's Trauma-Informed Model, FGC members speak to how components of the FGC's trauma-informed research and planning process were able to successfully facilitate partnership development and 
engage the Portland Native community within the FGC. Findings from this chapter provide valuable insight into the third research question. Additionally, Chapter 8: Findings on the Barriers to the FGC's Trauma-Informed Model reveals how the on-going systemic transmission of historical trauma by governmental agencies as well as the obstacles within the Portland Native community create barriers that influence the effectiveness of the FGC's trauma-model. These findings address both the second and third research questions of this study.

The concluding chapter provides discussion of partnerships between government agencies and the Portland Native community within the FGC, given the context of historical trauma and the FGC's trauma-informed model. This chapter also includes recommendations of strengths to improve upon and barriers to address within the Future Generations Collaborative. These findings are additionally situated into a larger body of theoretical and empirical literature. Finally, this chapter determines the implications of these findings as well as possibilities for future research. 


\section{Chapter 2: Background}

\section{Introduction}

This chapter provides relevant background into the relationships between government agencies and the Portland Native community within the Future Generations Collaborative (FGC). First, historical information is provided that addresses colonialism within Oregon and subsequent impacts on Native health and social inequalities. These historical events provide context for discussion of historical trauma within the Portland Native community throughout this case study. Second, this chapter outlines empirical research on the precedent of Fetal Alcohol Spectrum Disorder (FASD) initiatives within Native communities. In order to understand the discussion of the FGC's approach to addressing substance-exposed pregnancies, it is essential to situate the FGC's traumainformed model within these greater health trends. Third, this chapter establishes the specific setting of the FGC by outlining the details of the FGC's trauma-informed model, including the FGC's goals, membership roles, and the project timeline. Details from this section help to ground the discussion of the FGC's trauma-informed model throughout the remainder of this case study.

\section{Relationships between the Government and Portland Native Community}

The formation of the Future Generations Collaborative is situated in a legacy of unequal relationships between the Oregon government and the Portland Native community throughout the colonial history of the state. Portland is located in Multnomah County, OR and rests on the traditional homelands of the Clackamas Chinook and the 
Tualatin Kalapuya. In 2011, Portland State University and the Coalition of Communities of Color published a report, entitled The Native American Community in Multnomah County: An Unsettling Profile. This report details the historical relationship between Multnomah County and Native American people living in the area by documenting harsh inequalities from initial colonial contact that persist into the modern era (Curry-Stevens et al. 2011). The state of Oregon has a unique colonial history including genocide and forced removal of Native Americans from their ancestral lands. In 1953, the state of Oregon broke international law by the terminating several Oregon tribes as means of revoking tribal sovereignty and taking away millions of acres of land (Curry-Stevens et al. 2011). The state of Oregon has also been responsible for forced assimilation by boarding schools, and the coercive sterilization of thousands of Native American women (and men) as well as discrimination in voting (Curry-Stevens).

This history provide context for health and social disparities that exist in Portland, OR today. Curry-Stevens (2011) found that many health and social disparities are more severe for the Native community in Portland than in other parts of the country. For example, while nation-wide approximately $20 \%$ of Native American families experience poverty, nearly one-third of Native American families live in poverty in Portland, OR (Curry-Stevens et al. 2011). In 2009, the Oregon Population Survey found a large health insurance coverage gap with $23.6 \%$ of Native Americans uninsured compared to $14.6 \%$ of Whites (Curry-Stevens et al. 2011). This disparity may be explained by the fact that not all Native Americans are able to access benefits from Indian Health Services.

Indian Health Services (IHS) is the U.S. federal agency responsible for the provision of health services to members of federally recognized tribes. This trust 
responsibility was established through treaty agreements between the United States and sovereign Tribal Nations (Curry-Stevens 2011; Gurr 2011). According to Gurr (2011), chronic underfunding represents a failure on the part of the United States to meet internationally recognized treaty obligations with Native tribes. Furthermore, Native Americans who are not members of federally recognized tribes do not have access IHS and face further barriers to medical care (Curry-Stevens 2011; Gurr 2011). These outcomes demonstrate two examples of how persistence of cycles of inequality and oppression continue to affect the relationship between the Portland Native community and the Oregon government today.

These events also provide context for persistent efforts of local Native American activism that has achieved many hard-won victories such as restoration in the 1980's for six Oregon tribes and the establishment of 28 Native-driven organizations in the Portland area (Curry-Stevens 2011; Rosenthal 2002). Additionally, while the Coalition of Communities of Color acknowledges significant efforts by specific Multnomah County government agencies to address the needs of Native communities, they argue that much more is needed, stating:

But our needs are mounting at a profound rate. Tied to faltering education, employment, and housing systems leave too many poor, broken, and at risk of deteriorating health and well being. The resilience of this community is enormous (having been required to survive colonization and slavery), but ignoring policy action is understood to be a further act of colonization (Curry-Stevens 2011, p. $88)$.

Curry-Stevens (2011) highlights the ways in which present day social inequalities are situated within a legacy of colonization and continue to escalate the disparities experienced by Native communities. Further, government agencies neglect of social 
inequalities experienced within the Portland Native community today represents ongoing nature of colonization in the modern era.

\section{Native-Specific Fetal Alcohol Spectrum Disorder Health Initiatives}

The legacy of Native American oppression in Multnomah County creates context for ongoing concerns about substance-exposed pregnancies within the Portland Native community. Fetal Alcohol Spectrum Disorder (FASD) is an umbrella term that describes a range of diagnoses including Fetal Alcohol Syndrome (FAS), partial FAS, alcoholrelated birth defects, and alcohol-related neurodevelopmental disorders (Sarche et al. 2011). Native Americans experience the highest rates of alcohol use and dependence, and similarly the concern for FASD in Native Americans communities has been well documented (Sarche et al. 2011). Prevalence statistics from Indian Health Services demonstrate that Native American women are much more likely to drink during pregnancy with rates three times higher than all other races ( $4.5 \%$ versus $1.5 \%)$ and up to eight times the rate of all other races in certain IHS regions (Shalala et al. 1999).

FASD rates within Native communities provide another example of how health outcomes are situated within the ongoing history of colonialism (Sarche et al. 2011). However, many public health campaigns have neglected this historical context or the ongoing role of socioeconomic disenfranchisement when addressing FASD among Native Americans (Retner et al. 2012). These non-effective public health approaches rarely involve Native American stakeholders in their development and are instead grounded in negative representations of Native communities by "outside" researchers (Retner et al. 2012). In many ways, these initiatives have perpetuated stereotypes of the 
“drunken Indian” (Retner et al. 2012). Salmon's (2011) study of a Canadian FASD health initiative in First Nations communities uncovers how colonization is reified when States' neoliberal economic and political ideologies inform health efforts for indigenous populations while in partnership with decolonization movements. She argues, "FASD is a condition unparalleled in its complexity as both a public health issue and an expression of gendered and racialised conditions of disenfranchisement and abandonment"(Salmon 2011, p. 175). In this way, Native-driven prevention programs that seek to improve Native American women's health incrementally over an extended period of time may be blocked from funding and blamed for failure if they cannot concretely demonstrate short term results (Salmon 2011).

While these numerous obstacles shed light on the political enormity of FASD prevention programs in Native communities, it is also important to mention gains from successful Native health initiatives. Native Americans have the higher rates of abstention from alcohol than the general population (Sarche et al. 2011). Furthermore, the Center for Disease Control (2009) found that the rates of FAS deaths among Native Americans have decreased 19\% between 2002 and 2007. According to Retner et al. (2012) these successes can be attributed in part to culturally sensitive and multi-faceted FASD prevention programs that include Native Americans in the development and implementation of the initiative.

The Future Generations Collaborative's Trauma-Informed Model

The Future Generations Collaborative focuses on preventing substance-exposed pregnancies through the promotion of healthy pregnancies and healing from historical 
trauma within the Portland Native community. Through a trauma-informed research and planning process, the FGC's specific objectives include:

1. To gauge the Portland Native community's knowledge base of Fetal Alcohol Spectrum Disorder (FASD).

2. To obtain feedback from Native youth, adults, and elders on the impact of historical trauma within the urban Native community.

3. To examine community and cultural strengths to address healing and the promotion of healthy Native families.

4. To solicit ideas to further engage the Portland Native community to promote support, guidance, belonging, and giving back.

The FGC's objectives reflect a holistic Native-specific approach to addressing substanceexposed pregnancies and historical trauma within the Portland Native community. In order to accomplish these goals the FGC developed a trauma-informed community-based participatory research and planning model with two primary aims: (a) to build and improve upon collaborative relationships between stakeholders, including government agencies, Native organizations, non-governmental organizations and (b) to engage the Portland Native community in a culturally specific form of community-based participatory research and planning methods.

City Match, a national agency under the Center for Disease Control, provided initial funding for the FGC's trauma-informed research and planning process. Subsequent funding was obtained from the Northwest Health Foundation through a $\$ 50,000$ grant to support engaging Native communities in building maternal child health capacity. Additionally, the FGC received \$40,000 from Multnomah County, not including full-time equivalent pay toward employee workload or in-kind contributions toward organizational supports including meeting rooms, supplies, etc. Health Share of Oregon and the Oregon Health Authority also provided the FGC with grants of $\$ 91,000$ and $\$ 10,000$ respectively. 
Approximately $\$ 3,000$ of funding was also secured from the Native American

Rehabilitative Association of the Northwest (NARA) as well as a $\$ 1,000$ grant from the National Child Welfare Association.

There are two types of FGC members, including FGC partners as well as the Elders and Natural Helpers. FGC partners are members of the FGC who formally represent government agencies, Native-serving organizations, or other non-governmental organizations. For the most part, FGC partners from government agencies are non-Native while FGC partners from Native-serving organizations are Native American. Elders and Natural Helpers are a group of Native-identified men and women from the Portland Native community who have been trained to assist in the FGC by recruitment of participants for the community forums, co-facilitation of community forums and other community-based meetings, data analyses, dissemination of results and the action plan. Elders and Natural Helpers also participate in the larger organization of the FGC. A small number of Native FGC members inhabit the roles of both FGC partners and Elders and Natural Helpers. For the purposes of this study, the researcher counted FGC partners and Elders and Natural Helpers based on the FGC members' primary role during the time of participant observation. Both groups of FGC members meet regularly for the purposes of relationship building, training, planning, and preparation for FGC events.

The project timeline of the FGC initially included four phases. Phase I was focused on planning and preparation and included recruitment of FGC partners and partnership development. This phase extended from August of 2011 through April 2013. Phases II-IV describe the period of implementation where community capacity building is prioritized. Phase II was characterized by partnership building and community 
engagement and extended between September 2012 through May 2013. This phase also included the recruitment and training of Elders and Natural Helpers, conducting community forums, analyzing the data, and increasing organizational partnerships. Additionally, phase II included two "Meaning Making Meetings" which were two events serving as community validation for data produced during the community forums and where community permission was requested for use of the data.

Phase III was the action planning phase and was largely organized around the Gathering of Native Americans (GONA). GONA is an indigenous culturally centered curriculum for community partnership trainings focused on reducing and preventing substance abuse (Chino and DeBruyn 2006). This model was developed during the 1990's by a group of Native American health professionals under the Substance Abuse and Mental Health Services Administration's (SAMPSA) Center for Substance Abuse and Prevention (Chino and DeBruyn 2006). According to Chino and DeBryun (2006), the goals of the GONA are to “...provide training that offered hope, encouragement, skills transfer, and a positive basis for indigenous community action based on values inherent in traditional indigenous cultures (p. 597)". The FGC's GONA was a three-day event where results from the community forum were integrated into the culturally grounded curriculum and where an action plan was created to promote healthy pregnancies and healing from historical trauma within the Portland Native community. This planning period extended from June 2013 until January 2014 when the GONA was held. Finally, Phase IV is centered on finalizing an action plan and soliciting commitments from the Portland Native community as well as other governmental and non-governmental organizations. This phase also includes disseminating the action plan and formalizing 
leadership structures within the FGC for sustainability and community accountability. This phase began in July 2013 and continued past this case study's period of participant observation, which culminated in February of 2014.

\section{Conclusion}

The setting of this case study has been profoundly influenced by the legacy of colonization in Oregon, the persistent health inequities experienced by Native Americans in Multnomah County, and the national public health trends for addressing substanceexposed pregnancies. Additionally, detailed descriptions of the FGC's trauma-informed model are necessary for understanding the nuances of this case study. Thus the material provided in this chapter lays the groundwork for exploring historical trauma and the FGC's trauma-informed research and planning process. Background information also helps to establish a multi-layered context for understanding the complexities of partnerships between government agencies and the Portland Native community within the FGC. 


\section{Chapter 3: Literature Review}

Introduction

This chapter provides an overview of the previous scholarship in order to establish context for this case studies' analysis of the relationships between government agencies and the Portland Native community within the FGC. Previous research establishes the complexities of collaborative partnerships between government agencies and Native communities, especially given the profound influence of historical trauma. Therefore, theory of historical trauma is used as the basis of analysis throughout this case study. The current body of research that specifically explores Native American historical trauma does not emphasize government agencies' contemporary role in the systemic transmission of historical trauma. Thus tribal critical race theory and Myrdal's (1962) vicious cycle provide important theoretical tools for understanding the government's past and contemporary role in systemically perpetuating historical trauma.

In reviewing an interdisciplinary body of literature, this chapter outlines useful theoretical perspectives for situating the findings of this case study. These theories create a foundation for the analytical model that was developed inductively over the course of this case study. Empirical research on community-based participatory research and planning is further outlined in order to ground the FGC's trauma-informed research and planning within the existing literature. In this way, theoretical and empirical research provide context for the in-depth analysis in the following chapters as well as support the unanswered questions that this case study seeks to address.

Historical Trauma within Native Communities 
Theories of historical trauma are widely used within research in Native communities to describe the cumulative and intergenerational impacts of colonization on present generations of Native Americans (Brave Heart 1998; Evans-Campbell 2008; Fast and Collin-Vézina 2010). The theoretical roots of historical trauma can be found within the concept of intergenerational trauma. Intergenerational trauma first emerged within academia to explain trauma experienced by the decedents of Holocaust survivors (Fast and Collin-Vézina 2010). Brave Heart (1998) was the first to apply the concept of intergenerational trauma to Native communities, thus coining the term historical trauma. Since Brave Heart's (1998) landmark study, numerous Native scholars have contributed to the interdisciplinary body of research on historical trauma within Native communities.

Evans-Campbell (2008) describes three criteria for historical trauma, including (a) trauma is experienced by many people within a community, (b) traumatic events cause high levels of collective distress, and (c) traumatic events are imposed upon the community by outsiders with hostile and violent intent—often genocidal in nature. Numerous scholars have connected various U.S. and Canadian colonial policies to the creation of historical trauma between Native American and First Nations populations (Brave Heart 1998; Evans-Campbell 2008; Fast and Collin-Vézina 2010). Sotero (2006) describes these mass experiences of historical trauma as a process in which a dominant group subjugates a population through mechanisms of (a) segregation and displacements, (b) physical and psychological violence, (c) economic destruction, and (d) cultural dispossession. For example, coercive assimilation through Native American boarding schools represents an extremely traumatic governmental policy in both the United States 
and Canada.

During the boarding school era, Native children were removed from their families and their tribes and placed in boarding schools. Students were forced to participate in education which strived to "kill the Indian, save the man" by stamping out all Native culture and identity and instilling white-western culture and values. Boarding schools were incredibly violent and repressive institutions that systematically used mental, physical, and sexual abuse to achieve their colonial aims (Smith 2007). This historically traumatic period was enforced by U.S. and Canadian genocidal policies and impacted large populations Native Americans and First Nations people throughout the $19^{\text {th }}$ and $20^{\text {th }}$ centuries. Certainly, boarding schools were responsible for creating a high degree of collective distress and were also imposed upon by outsiders with culturally genocidal intent (Smith 2007). In this way, boarding schools meet all of Evans-Campbell's (2008) the criteria for creating historical trauma within Native communities.

Many other examples of governmental policies responsible for creating Native American historical trauma can be found throughout the literature (Brave Heart and DeBruyn 1998). Brave Heart and DeBruyn (1998) provide some important common examples of historical trauma experienced by Native Americans, including: genocide, removal to reservations, betrayal of treaties, armed conflict, the reservation system, as well as other assimilation policies such as the 1887 Dawes Allotment Act and termination during the mid $-19^{\text {th }}$ century. Historically traumatic events can also be culturally or tribally specific, such as the prohibition of whaling for Northwestern costal communities (Evans-Campbell 2008). Evans-Campbell (2008) describes how Native communities' experiences of historical trauma are broad and diverse, stating: 
Notably, such historically traumatic events are quite diverse, and, accordingly, the associated reactions are not comparable; trauma reactions stemming from witnessing a community massacre will likely be different from reactions to being forcibly removed from family to attend an Indian boarding school. However, the lens of historical trauma allows us to expand our focus from isolated events and their impacts to the compounding effect of numerous events over time (p. 321).

Demonstrated here is the ways in which various historically traumatic events compound over time. This highlights how U.S. policies spanning from initial colonial contact into the modern era have created widespread historical trauma for Native Americans. Once a historically traumatic period occurs, Evans-Campbell (2008) outlines two methods that transmit historical trauma interpersonally, including direct and indirect transmissions.

The direct method of transmitting historical trauma occurs as Native Americans are exposed to historical trauma through collective memory (Evans-Campbell 2008). Historical trauma is experienced vicariously throughout each generation who comes in contact with stories and oral traditions about historical trauma (Evans-Campbell 2008). In detailing the indirect method of the intergenerational transmission of historical trauma, Evans-Campbell (2008) describes how the initial experience of colonization by Native Americans creates a "trauma response" within multiple levels of Native American life, including Native individuals, families, and communities. This trauma response facilitates a break down in Native health and wellbeing at each level and can continue throughout the generations until the multi-faceted losses are restored (Evans-Campbell 2008). For example, trauma responses at the individual level could look like increased anxiety or substance abuse, while manifesting within families as impaired communication, and as loss of traditional practices within communities (Evans-Campbell 2008). EvansCampbell (2008) further describes how the multi-level trauma responses reinforce each 
other over the course of generations creating cumulative disadvantage in Native communities. In this way, trauma experienced through the initial act of colonization is compounded by trauma responses and passed down intergenerationally thus becoming historical trauma (Evans-Campbell 2008).

Researchers have also focused on the ways in which contemporary traumas experienced by Native communities interplay with historical trauma (Carter 2007; EvansCampbell 2008; Fast and Collin-Vézina 2010). Studies routinely demonstrate that Native Americans experience high levels of discrimination within contemporary society (EvansCampbell 2008). These experiences of discrimination are not only traumatic in their own right, but also situated within the greater legacy of historical trauma (Evans-Campbell 2008). Furthermore, Carter (2007) demonstrates how trauma rooted in the histories of colonization cannot be separated by experiences of discrimination and "race-based trauma" within the modern era. The overlap of historical and contemporary trauma suggests a societal transmission of historical trauma through contemporary traumas. Through this process historical trauma is compounded by contemporary acts of discrimination, thereby transmitting historical trauma through each generation of Native Americans who encounter discrimination.

In all cases, the transmission of historical trauma can be seen as a social process with devastating intergenerational impacts on Native American health. Canales et al. (2011) also describe how historical and contemporary traumas contribute to multigenerational mistrust between Native Americans and health care providers, thus deepening the impacts on Native American health. At the same time, academics that focus on historical trauma in Native communities also emphasize Native communities 
resilience in the face of historical trauma. In recent decades, researchers have produced an emerging body of work on resilience focused on a broad range of Native American health and social issues, from suicide rates to teen substance abuse (LaFromboise et al. 2006). According to LaFramboise et al. (2006), most research defines resilience as a process that develops "the capacity to face challenges and to become somehow more capable despite adverse experiences" (p. 194). In the context of historical trauma, protective factors serve as mechanisms that buffer against the impacts of historical trauma and facilitate resilience outcomes. Multiple dimensions of protective factors can be drawn from Native-specific resilience research, including: individual, family, community, and cultural protective factors (Kaplan et al. 1996; LaFromboise et al. 2006; Mohatt et al. 2004).

Walters and Simioni's (2002) theoretical contribution of the Indigenist Stress Coping Model is especially useful for understanding historical trauma's impacts on the relationships between government agencies and the Portland Native community. Walters and Simioni (2002) describe how historical trauma works alongside contemporary traumas to cause stress for Native Americans. The stress of all of these traumas combined to create direct impacts on the health outcomes for Native Americans (Walters and Simioni 2002). However, some of the stress created by historical trauma is mediated by cultural buffers, or protective factors, which facilitate coping and produce resilient health outcomes (Walters and Simioni 2002, see Figure 1). Within Walters and Simoni's (2002) model, numerous individual level protective factors are identified that help promote resilience. These protective factors include identity, attitudes, enculturation, as well as 
engagement in spiritual coping and traditional health practices (Walters and Simoni 2002).

Figure 1: Walters and Simoni’s (2002) Indigenist Stress Coping Model

Stress

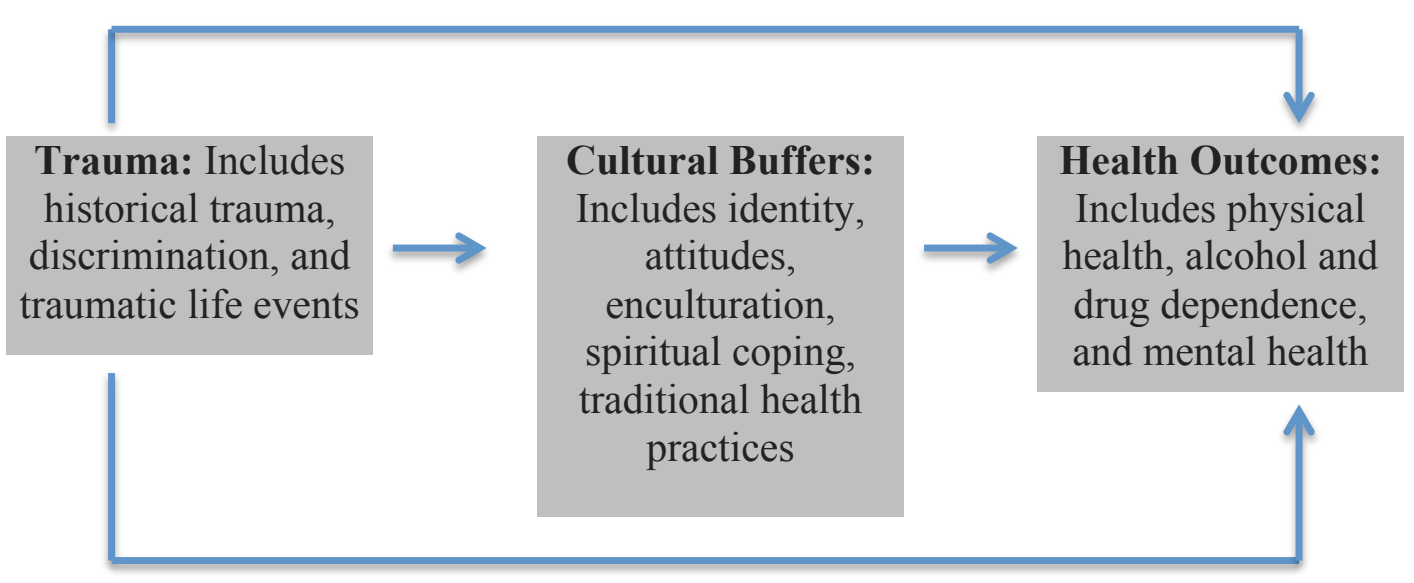

Scholars studying Native American resilience have also identified numerous examples of protective factors on the family, community, and cultural levels of Native American life (Kaplan et al. 1996; LaFromboise et al. 2006; Mohatt et al. 2004). Several studies demonstrate that close affectionate ties, extended support networks, positive family environments, and shared responsibilities, as well as positive parental modeling and coping skills are all examples of important protective factors within Native American families (Kaplan et al. 1996; LaFromboise et al. 2006; Mohatt et al. 2004). Similarly, protective factors have been found within social support, positive community norms, community resources, and self-determination within Native communities (Kaplan et al. 1996; Fast and Collin-Vézina 2010; LaFromboise et al. 2006; Mohatt et al. 2004). Finally, participation in traditional activities, as well as cultural and spiritual renewal represent examples of Native American cultural protective factors that promote healing 
from historical trauma and resilience (Kaplan et al. 1996; Fast and Collin-Vézina 2010; LaFromboise et al. 2006; Mohatt et al. 2004).

Similarly, Evans-Campbell's (2008) multi-level framework for understanding the impacts of historical traumas can be used to expand Walters and Simoni’s (2002) discussion of historical trauma and resilience. Evans-Campbell's (2008) work suggests examining the impacts of historical trauma from a broadened scope, to include impacts on Native American individual, family, and community health. This theoretical expansion aligns with Czyzewski's (2011) work on colonialism as social determinant of health. Czyewski (2011) traces not only historical trauma's direct impacts on Native health outcomes, but also historical traumas impacts on Native social determinants of health, such as: social, economic, political conditions among Native Americans. Whether used to examine historical traumas impacts on a specific Native health outcome or broadened to assess impacts on Native social determinants of health at large, Walters and Simoni's (2002) model provide an important theoretical insight into the phenomenon resilience from historical trauma.

The current body of research that specifically explores Native American historical trauma primarily focuses on the intergenerational transmission of historical trauma within Native communities. These previous studies often cite governmental agencies' policies and practices as the initial source of historical trauma within Native communities. Research that focuses on the societal transmission of historical trauma through contemporary traumas typically focuses on discrimination generally rather than specific to government agencies. Sotero (2006) suggests that psychosocial, legal and social discrimination, as well as social, economic, and political system can serve 
as modes of societal transmission that pass on historical trauma intergenerationally. However, Sotero (2006) also suggests that more research is needed to understand the specific mechanisms of intergenerational transmission. As follows, the current body of literature on historical trauma does not yet emphasize government agencies' contemporary role in perpetuating historical trauma. Tribal critical race theory works well with theories of historical trauma in order to explain how the persistent colonial influence contributes to a systemic transmission of historical trauma within Native communities by governmental agencies.

\section{Tribal Critical Race Theory}

Taken together, theories of historical trauma and tribal critical race theory provide a strong theoretical framework for understanding the relationships between government agencies and Native communities. Tribal critical race theory is based in Native American localized and historical epistemologies (McKinley and Brayboy 2005). This complements theoretical discussions of historical trauma by providing analysis of specific contexts in which Native Americans experience historical trauma. Moreover, tribal critical race theory helps to create space for acknowledging the diversity of historical trauma experiences among Native communities. Historical trauma and tribal critical race theory both focus on colonization as a persistent coercive force within Native American life. As described in the previous section, theories of historical trauma describe on-going colonization through various policies and practices that extend from initial colonial contact into the modern era (Brave Heart and DeBruyn 1998). The following description 
of tribal critical race theory adds to this critique by emphasizing how policies of the U.S.

government have perpetuated colonization over the long term.

McKinley and Brayboy (2005) contribute many relevant insights into the relationships between government agencies and Native communities. According to McKinley and Brayboy (2005), the nine tenets of tribal critical race theory are:

(1) Colonization is endemic to society. (2) U.S. policies toward Indigenous peoples are rooted in imperialism, white supremacy, and a desire for material gain. (3) Indigenous peoples occupy a liminal space that accounts for both the political and racialized natures of our identities. (4) Indigenous peoples have a desire to obtain and forge tribal sovereignty, tribal autonomy, self-determination, and self-identification. (5) The concepts of culture, knowledge, and power take on new meaning when examined through an Indigenous lens. (6) Governmental policies and educational policies toward Indigenous peoples are intimately linked around the problematic goal of assimilation. (7) Tribal philosophies, beliefs, customs, traditions, and visions for the future are central to understanding the lived realities of Indigenous peoples, but they also illustrate the differences and adaptability among individuals and groups. (8) Stories are not separate from theory; they make up theory and are, therefore, real and legitimate sources of data and ways of being. (9) Theory and practice are connected in deep and explicit ways such that scholars must work towards social change (p. 429-430).

Notably, many of these tenets highlight the systemic ways governmental agencies use policies to further the project of colonialism in the modern era. Imperialistic, white supremacist, and neoliberal enabling ideologies shape these policies and support the continued colonization of Native Americans (McKinley and Brayboy 2005).

Additionally, many governmental policies within Native communities, including policies that secure social services for Native communities, are actually rooted within the colonial project of assimilation (McKinley and Brayboy 2005). Several scholars similarly highlight this connection through discussions of how governmental policies for assimilation — or "civilization" — of Native Americans have actually been designed to promote cultural genocide within Native communities (Brave Heart and DeBruyn 1998, 
Churchill 2004). Similarly, governmental agencies lack of follow through in implementing policies for the for the betterment of Native communities — such as failing to the fulfill treaty obligations of providing health and social services to Native Americans - represents another way government agencies perpetuate colonization (Curry-Stevens et al. 2011). In this way, many governmental policies and the enabling ideologies that support them are incompatible with the health and wellbeing of Native Americans.

Throughout McKinley and Brayboy's (2005) theoretical framework other prominent themes of self-determination, tribal autonomy, and sovereignty emerge. These themes mirror the discussion of Native protective factors at the community level that help to promote resilience from historical trauma (Fast and Collin-Vézina 2010). Within the context of government-to-government relationships between the United States and federally recognized tribes, self-determination, tribal autonomy and sovereignty often carry legal bearing. However, in the urban setting — where the majority of Native Americans live in the $21^{\text {st }}$ century - these terms become complicated (Beck 2002).

Since urban Native communities lack the legal protections available to tribes, selfdetermination means the ability to determine the social, political, economic, and spiritual direction of one's own community (Beck 2002). Beck (2002) describes selfdetermination, stating: "To an urban Indian community, it means that community's ability to define itself and its needs and its ability to advocate for itself in the larger society under its own terms. It implies the development of voice through which all of these things can be done" (p. 118). Importantly, Beck's (2002) emphasis on urban Native communities" ability to advocate for itself "under its own terms" and through the 
“development of voice” parallels McKinley and Brayboy’s (2006) attention to the importance of Native culture, tradition, and epistemologies.

In order to engage in self-advocacy and achieve autonomy, Native communities must have the ability to represent themselves according to their own values and interests within relationships with governmental agencies (McKinley and Brayboy 2005). Furthermore, this engagement must be shaped by Native-driven processes and reflect Native culture, tradition, and epistemologies (McKinley and Brayboy 2005). Otherwise, the status quo of endemic colonization will persist within U.S. society (McKinley and Brayboy 2005). Scholars have also suggested that Native representation, Native-driven processes, and Native epistemologies are all essential in order to promote the decolonization and self-determination in Native communities (Grande 2004; Smith 1999). Therefore, tribal critical race theory emphasizes Native resistance for its ability to promote adaptability within Native communities, or resilience to colonization by governmental agencies. Native resistance also serves to push back against the government's continued role in colonization. Fortunately, Native American activism has already achieved many hard won gains in terms of resisting colonialism and creating a legacy of self-determination within Native communities (Johnson, Nagel, and Champagne 1997).

Tribal critical race theory's discussion of the endemic nature of colonization within governmental agencies is suggestive of the complex ways in which governmental agencies perpetuate historical trauma through modern acts of colonization. Discussion of how governmental policies and enabling ideologies perpetuate colonization fits well with historical trauma theorists' description of the social transmission of historical trauma 
through contemporary traumas. Applying Myrdal's vicious cycle (1962) to tribal critical race theory and theory of historical trauma lends useful sociological insight into the endemic nature of colonialism by helping to tease out possible mechanisms that government agencies use to perpetuate colonialism and historical trauma within Native communities.

Myrdal's Vicious Cycle

While Myrdal's (1962) theory was initially created to explain the long-term subordinated status of African Americans, it can also be used to explain the persistence of historical trauma and colonization in relationships between government agencies and Native communities. Myrdal (1962) describes how African Americans were initially subordinated by the U.S. system of slavery. This parallels Sotero's (2006) discussion of how majority groups subjugate a population creating a first generation that is impacted by historical trauma. Therefore, Myrdal's (1962) description of the initial subordination of African Americans under slavery draws distinct parallels to U.S. colonial projects within Native communities.

After slavery ended, Myrdal (1962) describes the ways in which a racial caste system is maintained intergenerationally through a "vicious circle of cumulative causation" (p. 208). This racial caste system is evidenced by the persistent social inequalities experienced by African American communities (Myrdal 1962). The racial caste system could also be described in terms of structural inequalities that continue the legacy of subordination. Similarly, tribal critical race theorists highlight the ways that colonization is embedded into contemporary governmental policies, therefore 
contributing to the structural inequalities experienced by Native communities. Application of historical trauma theory might also suggest that structural inequalities represent a site where the historical trauma from the initial incidences of colonial subordination is compounded for Native Americans by the contemporary traumas of ongoing social inequalities.

Myrdal (1962) discusses how white prejudice and discrimination work together to reinforce the low standards of living experienced by African American communities. White racism occurs when members of the majority group see the social inequalities experienced by African Americans and makes the opportunistic assumption that these inequalities are natural (Myrdal 1962). Myrdal (1962) states, "Through the generations, strengthened by tradition and community consensus, a public opinion among whites is formulated which is plainly opportunistic in the interest of the majority group" (p.102). In this way, white racism can accumulate overtime while rationalizing both white privilege and African American disadvantage within the structural inequalities. Applying tribal critical race theorist's descriptions of enabling ideologies role in perpetuating colonization suggests a broadening of white prejudice to also include imperialistic and neoliberal enabling ideologies (McKinley and Brayboy 2005).

Myrdal's (1962) theory also suggests that enabling ideologies, such as white prejudice, insidiously create an environment where discrimination is prevalent. Describing economic discrimination against African Americans, Myrdal (1962) states, "The very fact that there is economic discrimination constitutes an added motive for every individual white group to maintain such discriminatory practices. Discrimination breeds discrimination" (p.381). This helps to explain how pervasive discrimination 
contributes to contemporary race-based traumas experienced by Native communities. Finally, Myrdal (1962) argues that discrimination deepens the subordinated status of African Americans, creating a vicious downward cycle that exasperates the structural inequalities experienced by African American communities. Therefore, the long-term subordinated status of African Americans is explained by the mutually reinforcing mechanisms of structural inequalities, enabling ideologies, and discrimination (Myrdal 1962).

Figure 2: Myrdal's (1962) Vicious Cycle

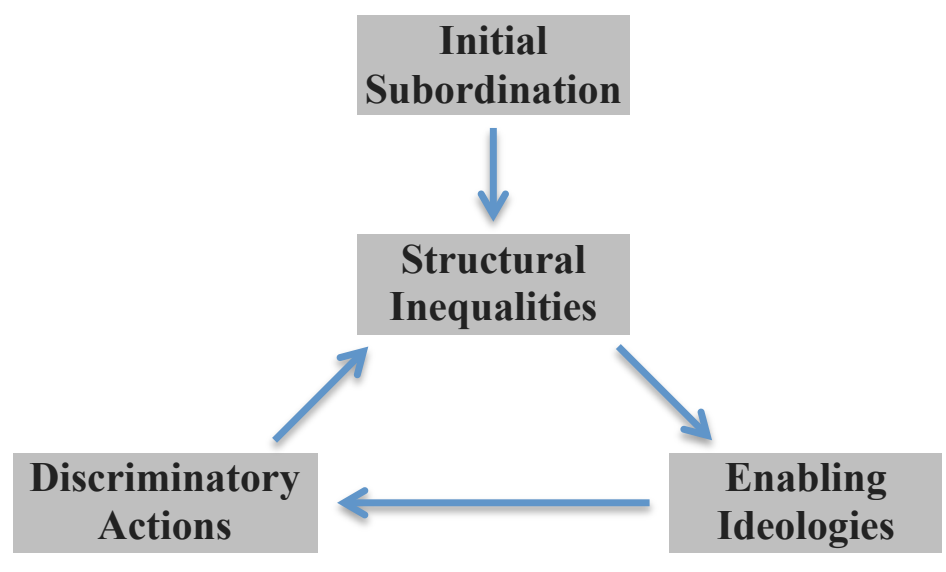

Myrdal (1962) explains how the interdependence of these mechanisms contributes to principle of accumulation, otherwise known as the vicious cycle. He states:

If either of the factors changes, this will cause a change in the other factor, too, and start a process of interaction where the change in one factor will continuously be supported by the reaction of the other factor. The whole systems will be moving in the direction of primary change, but much further. This is what is meant by cumulative causation.

Myrdal (1962) emphasizes how the interlocking mechanisms of structural inequalities, enabling ideologies, and discrimination not only maintain the subordination of African Americans, but also continuously compound the social inequalities experienced African 
Americans over the long-term. Myrdal (1962) also describes how the process of mutual interaction can spiral upwards, thus improving the social conditions of African Americans by decreasing enabling ideologies, discrimination, and structural inequalities. Significantly, the mechanisms of vicious cycle are so deeply intertwined that any reform movement to change the direction toward an upward spiral would require significant change toward all three mechanisms (Myrdal 1962).

Myrdal's (1962) description of the cumulative nature of the interlocking mechanisms of long-term subordination supports McKinley and Brayboy’s (2006) discussion of the endemic nature of colonization. Additionally, each of Myrdal's (2962) mechanisms could be viewed a site of contemporary trauma that acts to perpetuate historical trauma within Native communities (Evans-Campbell 2008). This would suggest that the interlocking mechanisms of Myrdal's vicious cycle could play a role in the societal transmission of historical trauma. Tribal critical race theory's emphasis on the government's role in perpetuating colonization supports examining government agencies' role in systemically perpetuating historical trauma and transmitting it to generations of Native Americans through the interlocking mechanisms of Myrdal's (1962) vicious cycle.

\section{A New Analytical Model for Understanding the Historical Trauma Process}

This chapter has so far outlined the key theories that inform this case study's analysis. Drawing from both theoretical perspectives and the data from this case study, I developed an analytical model to illustrate historical trauma as a social process (see Figure 3, p. 32). This figure integrates the findings of this case study with the theoretical perspectives of (a) historical trauma, (b) tribal critical race theory, and (c) Myrdal's 
(1962) vicious cycle. The model serves to explain the government's role in creating and sustaining historical trauma within Native communities. Additionally, the model provides essential context for understanding FGC members' evaluation of government relationships with the Portland Native community within the FGC. Further discussion of how the data from this case study informs the theoretical model can be found in Chapter

\section{4: Methods.}

Figure 3: The Historical Trauma Process

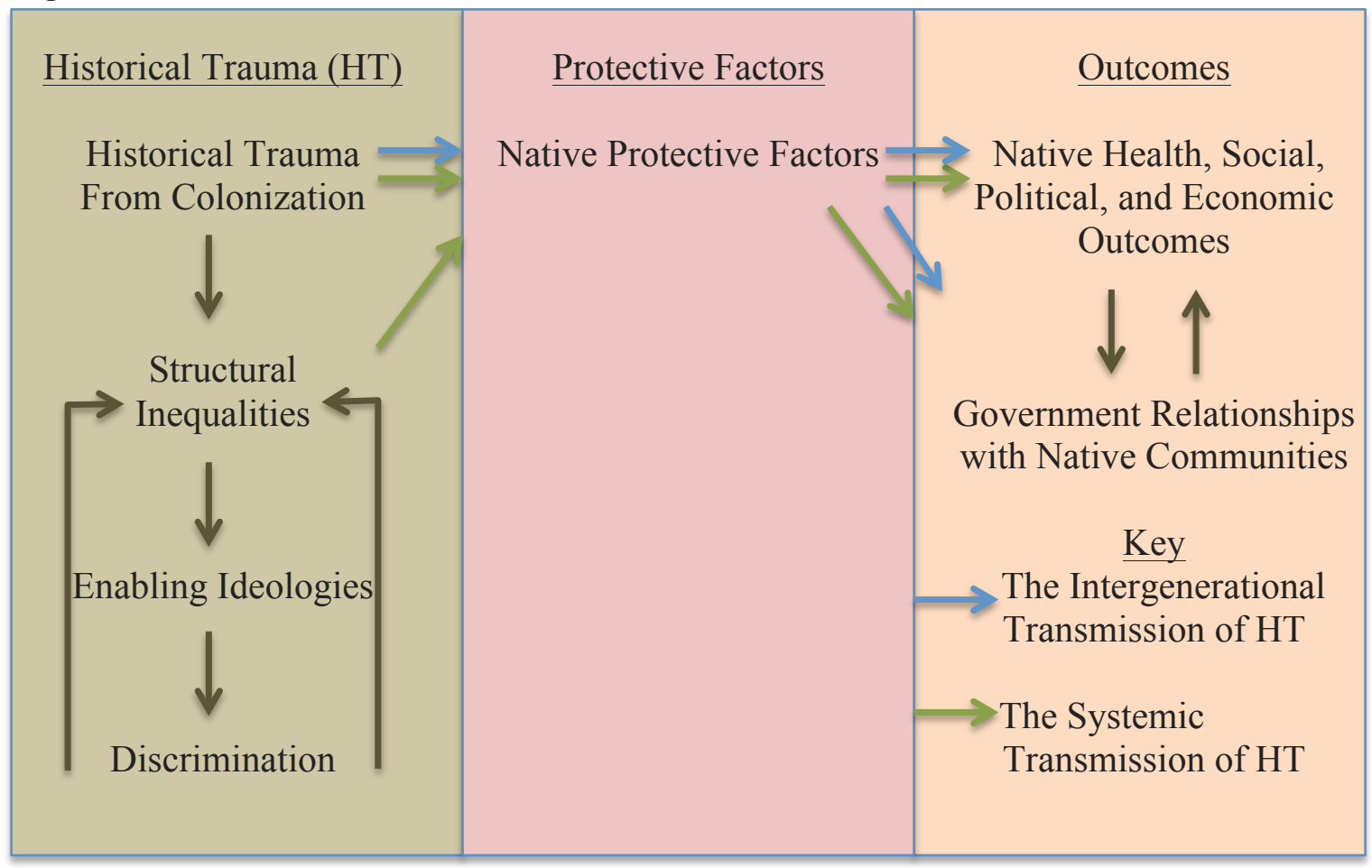

The analytical model presented above is in keeping with Evans-Campbell's (2008) description of the direct and indirect intergenerational transmission of historical trauma. First, the model suggests that periods of historical trauma occur as a result of colonial projects that extend from initial colonial contact into the modern (EvansCampbell 2008). Numerous historical trauma theorists suggest that the intergenerational 
transmission of this historical trauma has profound impacts on Native health outcomes (Brave Heart and DeBruyn 1998; Evans-Campbell 2008, Fast and Collin-Vézina 2010; Walters and Simoni 2002). Additionally, Czyewski's (2011) work suggests that historical trauma impacts the social determinants of Native health, including Native social, political, and economic outcomes. Walters and Simioni's (2002) indigenous stress coping model is also reflected in the model's emphasis on how protective factors mediates the influence of this intergenerational transmission and helps to explain resilient outcomes in terms of Native health.

Theories of historical trauma, tribal critical race theory, and Myrdal's vicious cycle support examining the government's role in creating the initial historically traumatic events that give way to this process of intergenerational transmission (Brave Heart and DeBruyn 1998; Churchill 2004; McKinley and Brayboy 2005; Myrdal 1962; Sotero 2006). Application of Canales et al.'s (2011) theory of multi-generational mistrust could be used to suggest that if Native communities find the government responsible for creating the initial incidence of historical trauma, then mistrust would become a significant outcome of the historical trauma process. Canales et al.'s (2011) work could also be used to suggest that the relationships between government agencies and Native communities and Native health status would mutually reinforce each other as outcomes of the historical trauma process.

Furthermore, theories of historical trauma, tribal critical race theory, and Myrdal's vicious cycle provide backing for the examination of government agencies potential role in the systemic transmission of historical trauma (Evans-Campbell 2008; McKinley and Brayboy 2005; Myrdal 1962; Sotero 2006). The model suggests that historical trauma is 
embedded within the structural inequalities of governmental agencies (McKinley and Brayboy 2005; Myrdal 1962; Sotero 2006). Myrdal's (1962) vicious cycle could be used to describe a systemic process in which structural inequalities, enabling ideologies, and discriminatory actions of government agencies represent interlocking mechanisms that continually build upon each other. In this way, historical trauma could become subject to the process of accumulation as the initial historical trauma is compounded by contemporary traumas produced by governmental agencies within the vicious cycle (Evans-Campbell 2008; McKinley and Brayboy 2005; Myrdal 1962; Sotero 2006).

Historical trauma theorists' work on the societal transmission of historical trauma through contemporary traumas (Evans-Campbell 2008; Fast and Collin-Vézina 2010; Sotero 2006) could also be used to suggest that historical trauma is transmitted systemically as Native Americans encounter the contemporary traumas caused by the vicious cycle of governmental structural inequalities, enabling ideologies, and discrimination (Myrdal 1962). The systemic transmission of historical trauma would then likely follow the same process as the intergenerational transmission of historical trauma (Evans-Campbell 2008; Walters and Simoni 2002). Thus the systematic transmission of historical trauma could be expected to face some mediation by Native protective factors, yet still create impacts in terms of Native health outcomes (Walters and Simoni 2002) and government relationships with Native communities. Again, application of Canales et al.'s (2011) research on multi-generational mistrust would suggest that if the government was found responsible for both creating and sustaining historical trauma through this systemic process, then high levels of mistrust could be expected in the relationships between government agencies and Native communities. 
This chapter has focused on building a theoretical framework that supports the analytical model for understanding the government's role in systemically transmitting historical trauma. Empirical studies of community-based participatory research are also useful for explaining how historical trauma and the FGC's trauma-informed research and planning process affect the relationships between government agencies and the Portland Native community within the FGC. The next section will discuss how community-based participatory research can serve as a protective factor for Native communities. In this way, the FGC's community-based participatory research and planning process could be potentially situated within this analytical model as another protective factor. Therefore, the FGC's trauma-informed model might mediate the impacts of the historical trauma process on Native health outcomes and the relationships between government agencies and Native communities.

\section{Community-Based Participatory Research in Native Communities}

The history of research within Native communities reveals a site of unequal relationships that have been historically harmful to Native communities (Brayboy and Deyhle 2000). "Drive-by" research has become known as common place within Native communities where non-Native researchers enter Native communities, extracting information that furthers their own careers while leaving Native communities with nothing (Thomas et al. 2011; Wallerstein and Duran 2008; Walters et al. 2009). Misrepresentation of Native Americans has also been prevalent by "outsider" researchers who have perpetuated colonial "othering" through practicing eurocentrism throughout the research processes (Mihesuah 2003). Brayboy and Deyhle (2000) likewise argue that 
many western research methods, tools of analysis, and ways of reporting data are in opposition to Native American epistemologies and practices. In this way, culturally inappropriate research can again contribute to misrepresentation of Native communities. Native American mistrust of research is also explained by the prevalence of culturally inappropriate research methods (Brayboy and Deyhle 2000).

There exists a large body of literature that establishes community-based participatory research (CBPR) as an important tool for conducting transcultural research with Native communities (Holkup et al. 2004; Strickland 2006; Thomas et al. 2011; Wallerstein and Duran 2006; Wallerstein and Duran 2008; Walters et al. 2009). In the past several decades, CBPR has attracted attention as a research paradigm that challenges positivist notions of objective science and troubles the traditional Western researchersubject relationship (Wallerstein and Duran 2008). Drawing from Israel et al. (1998), Strickland (2006) describes seven key principles of CBPR:

(1) It recognizes the community as the unit of identity, (2) it facilitates collaborative, equitable partnerships in all phases of the research, (3) it promotes co-learning and capacity building among partners, (4) it emphasizes local relevance of public health problems and attends to multiple determinants of health, (5) it involves a systems development through a cyclical and iterative process, (6) information is disseminated to all partners and all partners participate in disseminations, and (7) it involves a long-term process and commitment (Pp. 231).

The body of literature on CBPR in Native communities validates that all seven of these principles are important specifically within this population.

The implementation of these principles has been found to promote authentic relationships between the researcher and subject, encouraging reciprocity in research, strengthening rapport, empowering communities, and helping to facilitate culturally 
appropriate methods (Holkup et al. 2004; Strickland 2006; Wallerstein and Duran 2008). While CBPR certainly has its advantages, researchers have also found unique challenges to the CBPR approach including scientific, ethical, structural, and interpersonal issues (Holkup et al. 2004; Strickland 2006). In this way, evaluation of CBPR approaches is essential for addressing challenges and determining best practices of CBPR for health promotion in Native communities (Springett and Wallerstein 2008). While a growing number of community-based participatory research methodologists are concentrating on the collaborative partnerships between universities and Native communities, there exist large gaps in the literature when it comes to examining the research and planning partnerships between government agencies and Native communities.

Wallerstein and Duran's (2006) make an important contribution to academic understandings of how Native American health research is inherently political, and may have harmful impacts on the Native community if the principles of CBPR are neglected. Wallerstein and Duran (2006) also highlight transformative capacity of CBPR, not only in producing evidence to change the health outcomes of Native Americans directly, but also by creating policy changes that includes Native American participation and vision in the policy creation process. In finding that community-based participatory research can be empowering for Native communities, the authors suggest that the research process itself can create social change in Native communities (Wallerstein and Duran 2006). Smith (1999) has likewise suggested that the process of decolonizing research methodologies can disrupt imperialism within academic settings by challenging the ways 
knowledge is created and used to inform policy and by including greater indigenous representation.

The majority of CBPR projects take place within the context of collaborative partnerships between academic research and Native communities. One example of a CBPR project is the Healing of the Canoe Project based on partnerships between the Suquamish tribe and the Alcohol and Drug Abuse Institute at the University of Washington (Thomas et al. 2011). Through a mixed methods process of interviewing key stakeholders, conducting focus groups, and meeting with Tribal Council and other community members, the Healing of the Canoe Project identified youth substance abuse and lack of cultural identity as a primary concern of the Suquamish tribe (Thomas et al. 2011). The collaboration was then able to work together to identify an evidence-based substance abuse prevention intervention and adapt it to ensure cultural appropriateness for the tribe (Thomas et al. 2011). The Healing of the Canoe Project engaged in evaluation practices to determine the quality of partnerships between the Suquamish tribe and the University of Washington (Thomas et al. 2011). While the Healing of the Canoe Project garnered many insights into the best practices of CBPR within tribal communities, the primary lesson was the importance of trust and respect within the partnerships between Native American tribes and universities, as well as the importance of relying on Native community member expertise (Thomas et al. 2011).

Strickland (2006) provides another critical discussion of CBPR drawn from her experiences in implementing qualitative research with Pacific Northwest tribes in two studies over a 10-year period. Both studies were aimed at research and planning to improve Native American women's cancer screening behaviors (Strickland 2006). 
Strickland's (2006) research finds that CBPR is a culturally appropriate methodology for research within Pacific Northwest tribes. Additionally, Strickland (2006) also uncovers numerous institutional and structural barriers barriers to CBPR. For example, funding is needed to build relationships and establish trust throughout the research process (Strickland 2006). Therefore, lack of funding can serve as an important obstacle for effective community-based participatory research. While the body of research on CBPR provides deep insight into the relationships between academic institutions and Native communities, there exists a large gap in the literature when it comes to explaining CBPR partnerships between government agencies and Native communities. This case study aims to address this gap by focusing on the of the collaborative partnerships between government agencies and the Portland Native community within the FGC.

\section{Conclusion}

The interdisciplinary body of research presented in this chapter supports this case study's analysis of the relationships between government agencies and the Portland Native community within the FGC. By outlining the theoretical frameworks of historical trauma, tribal critical race theory, and Myrdal's vicious cycle, I develop an analytical model for understanding the government's role in creating and sustaining historical trauma within Native communities. This analytical model will also be used to address how historical traumas affects the partnerships between government agencies and the Portland Native community within the context of the FGC. Finally, the analytical model will be applied in conjunction with empirical research on CBPR for understanding how 
the FGC's trauma-informed research and planning process affects the relationships between government agencies and the Portland Native community within the FGC. 


\section{Chapter 4: Methods}

Research Design

The design of this case study allowed for the data collection on the complex partnerships between government agencies and the Portland Native community within the context of the FGC. More specifically, this design generated data to refine and address this case study's research questions. I employed mixed methodology in order to collect data including in-depth interviews, participant observation, and historical document analysis. In-depth interviews allowed for research centered on the participants' subjective experiences by creating space for FGC members' voices to be heard. Moreover, incorporating supporting data from participant observation and historical document analysis adds rich detail on the context of the specific phenomenon with the FGC. CBPR methods were also incorporated within this study's research design. In this way, the case study modeled many aspects of its design after the FGC's trauma-informed research process for addressing historical trauma and substance-exposed pregnancies within the Portland Native community (see Chapter 2: Background, p. 7).

\section{Researcher's Role within the Future Generations Collaborative}

In February 2013, I was invited to join the Future Generations Collaborative and to conduct an evaluation of their CBPR approach. This invitation was extended to me based on my relationship with an established Native academic. Additionally, I am the wife of a Native man from a local tribe and therefore have strong connections and commitment to the Portland Native community. FGC members also recognized the potential for reciprocity and community participation within my proposed research 
design. I was then invited to attend regular FGC planning meetings as well as any event connected to the implementation of the FGC's research and planning. The FGC provided me additional access to all relevant historical documents. My participation in the FGC helped to establish rapport within the collaborative and informed the development of my research questions, design and interview guide. Participation in the FGC also informed the analysis of this case study.

My status as the wife of a tribal member and my connection to the Portland Native community was instrumental in receiving access to the Future Generations Collaborative. Without such a status, it is not likely that I would have been invited to participate in the FGC and perform an evaluation as readily as I was. Therefore, when appropriate — such as during introductions when FGC members and Native community members were asked to identify by their name and tribe - I identified as "Amanda Mercier, I am white, Jewish, and my husband is Grand Ronde". After establishing initial contact with FGC members, I also participated in as many FGC events and Native community events as possible in order to build rapport. Within the FGC, I was thought of as an FGC partner and sometimes a Natural Helper based on my active level of participation within both groups of FGC members.

Some researchers might argue that a high level of participation in the FGC may open up this study to issues objectivity. Based on feminist and community-based research methodologies as well as my own experience within the FGC, this approach was absolutely critical for building trust. My status as an FGC member may also created the possibility that FGC members might only share experiences that reflected positively on the FGC. Therefore, I was sure to remind interview participants that their responses were 
confidential and were useful to inform future work with the Portland Native community. During interviews I also described this case study as an evaluation or inventory of what was working well and what needed improvement within the FGC. For the most part, the fact that the FGC invited me to conduct this research and participate throughout the research process helped strengthen rapport and build trust among FGC members.

\section{Data Collection}

This study relies on mixed qualitative methods for my primary and secondary collection methods. Briefly, the primary data collection method in this study was in-depth interviews. Secondary data collection methods included participant observation and historical document analysis.

\section{In-depth interviews}

In-depth semi-structured interviews with FGC members were the primary data collection method in this case study. The purpose of in-depth interviews was to gain a nuanced understanding of how FGC members evaluated partnerships between government agencies and the Portland Native community within the FGC. Whereas conventional evaluations rely on external experts, in-depth interviews privilege FGC member's subjective experiences and thereby create space for cooperation and collaboration. A preliminary interview guide was constructed drawing from the findings of Chino and DeBruyn (2006) and Walters and Simoni's (2009) in addition to FGC member feedback and historical documents. As part of my CBPR process, the interview 
guide was then brought to FGC members for feedback and revision, producing a refined and more culturally appropriate interview guide (see Appendix A, p. 178).

Table 1: Description of Interview Sample

\begin{tabular}{|c|c|c|c|}
\hline $\begin{array}{l}\text { Descriptive } \\
\text { Characteristic }\end{array}$ & \multicolumn{3}{|c|}{$\begin{array}{l}\text { Sample Percent, Sample Number }(\mathrm{N}=19) \text {; } \\
\text { Approx. Population Percent, Population Number }(\mathrm{P}=40)\end{array}$} \\
\hline FGC Role & $\begin{array}{l}\text { Elders and Natural } \\
\text { Helpers }\end{array}$ & FGC Partners & \\
\hline Sample & $47 \%, \mathrm{n}=9$ & $53 \%, n=10$ & \\
\hline Approx. Population & $11 \%, n=5$ & $89 \%, n=35$ & \\
\hline Race & Native American & $\begin{array}{l}\text { European } \\
\text { American }\end{array}$ & $\begin{array}{l}\text { Non-Native } \\
\text { Person of Color }\end{array}$ \\
\hline Sample & $74 \%, n=14$ & $16 \%, n=3$ & $10 \%, \mathrm{n}=2$ \\
\hline Approx. Population & $75 \%, n=30$ & $17 \%, n=7$ & $8 \%, n=3$ \\
\hline Gender & Male & Female & \\
\hline Sample & $11 \%, n=2$ & $89 \%, \mathrm{n}=17$ & \\
\hline Approx. Population & $13 \%, n=5$ & $87 \%, n=35$ & \\
\hline Parental Status & No Children & Children & $\begin{array}{l}\text { Children } \\
<3 \text { Years Old }\end{array}$ \\
\hline Sample & $16 \%, n=3$ & $84 \%, n=16$ & $42 \%, n=2$ \\
\hline Approx. Population & $\mathrm{n} / \mathrm{a}$ & $\mathrm{n} / \mathrm{a}$ & $\mathrm{n} / \mathrm{a}$ \\
\hline Age & Elder & $\begin{array}{l}\text { Adult } \\
>30 \text { Years Old }\end{array}$ & $\begin{array}{l}\text { Adult } \\
<30 \text { Years Old }\end{array}$ \\
\hline Sample Total & $16 \%, n=3$ & $58 \%, \mathrm{n}=11$ & $26 \%, n=5$ \\
\hline Approx. Population & $22 \%, n=9$ & $50 \%, n=20$ & $28 \%, n=11$ \\
\hline Employment Status & Employed & Unemployed & \\
\hline Sample Total & $68 \%, \mathrm{n}=13$ & $32 \%, \mathrm{n}=6$ & \\
\hline Approx. Population & $72 \%, n=10$ & $28 \%, n=11$ & \\
\hline $\begin{array}{l}\text { Agency of } \\
\text { Employment }\end{array}$ & $\begin{array}{l}\text { Government } \\
\text { Agency }\end{array}$ & $\begin{array}{l}\text { Native-Serving } \\
\text { Organization }\end{array}$ & $\begin{array}{l}\text { Other } \\
\text { Organization }\end{array}$ \\
\hline Sample Total & $26 \%, n=5$ & $37 \%, \mathrm{n}=7$ & $5 \%, \mathrm{n}=1$ \\
\hline Approx. Population & $25 \%, n=10$ & $38 \%, n=15$ & $10 \%, n=4$ \\
\hline
\end{tabular}

In-depth interviews were also important for collecting rich data on possible positive and negative evaluations by FGC members in confidentiality. As an invited researcher, I engaged in participant observation throughout the planning and implementation of their trauma-informed process, and therefore was able to established strong relationships within the FGC. I feel confident that I was able to bridge most 
insider-outsider issues in conducting research, thus providing me access to interviews with FGC partners as well as Elders and Natural Helpers. Several FGC members provided feedback into my methods for establishing rapport and reported that I had achieved a high degree of trust within the FGC.

At the same time, I had stronger relationships with FGC members who were willing to participate in interviews than FGC members who did not respond to recruitment. These FGC members tended to be more involved within the FGC's traumainformed process, thus creating increased opportunities for relationship development and trust building. During interviews, I reminded participants that any experiences they had within the FGC, both positive and negative, were valuable to the evaluation and that their interviews would remain confidential. Most participants had positive experiences within the FGC and favorable evaluations of the FGC's work in the Portland Native community. At the same time, several participants were also able to express concerns about the FGC's work and describe negative experiences within the FGC.

Based on estimates from internal FGC documents, the FGC's total population included approximately 40 total FGC members during the time of my participant observation. Purposeful sampling based on established rapport and in an effort to establish a representative sample was used to send out 30 recruitment emails for in-depth interviews. The study achieved a $63 \%$ response rate with interviews from 10 FGC partners and 9 Elders and Natural Helpers. Based on the estimated participant characteristics of the FGC as a whole, I was able to achieve a strong representative sample in terms of gender, race, and agency of employment (see Table 1, p. 43). This was significant considering the fact that race and agency of employment were the two 
participant characteristics of highest theoretical importance. The sample also contains satisfactory levels of representation in terms of age and employment status.

Elders and Natural Helpers maintain a disproportionately high representation within the sample. However, this decision was made intentionally in an effort to include the perspectives of Elders and Natural Helpers as members of the FGC and of the Portland Native community. There was not enough data available to estimate the representativeness of FGC members' parental status. Based on participant observation, I suspect that FGC members with children less than three-years-old were overrepresented within the sample. Since the level of FGC members' participation changed over time, this information was difficult approximate and therefore not included within Table 1. Based on participant observation, it is likely that this case study over represents very active FGC members and underrepresents inactive FGC members.

Participants were interviewed at a location of their choice, including: several Portland Native community centers, coffee shops, restaurants, parks, as well as participants' homes and workplaces. Native participants were each given a small, culturally appropriate gift of a handmade Columbia River style dentalium necklace. NonNative participants were given small gifts of journals and candy. Participants were also offered transportation and food when appropriate. This included bringing sack lunches to interviews and offering to pick up the check at restaurants and coffee shops. Occasionally, Native FGC members senior to me offered to buy me food or drinks during interviews. I accepted these offers based on my understanding of culturally appropriateness. While hand-transcription is the preferred culturally appropriate method within the FGC, FGC members supported my use of tape recorders during interviews. 
This was due to a lack of feasibility in simultaneously facilitating interviews and handtranscription. Additionally, confidentiality and trust issues were associated with inviting an additional researcher to hand-transcribe interviews.

\section{Participant Observation}

As a secondary research method, I also engaged in participant observation of FGC's trauma-informed research and planning process through attendance of various meetings and events. Participant observation at FGC meetings included the FGC's monthly meetings, specific task force meetings, GONA planning meetings, work retreats, as well as a few Elder and Natural Helper meetings. Additionally, I engaged at event participant observation during the trainings of Elders and Natural Helpers, the FGC's community forums, Meaning Making Meetings, and the GONA.

In participating in these events, I gathered contextual data, was able to collect data in the natural settings, and document phenomenon relevant to this study's research questions (Marshall and Rossman 2011). Furthermore, participant observation continued my face-to-face interaction with FGC members, thus helping to establish rapport within the FGC. Participant observation also provided opportunities for triangulation and clarification of complex interactions (Marshall and Rossman 2011). The participant observation period of my research extended one year, from February 2013 through January 2014. Since February 2014, I have continued participating within the FGC although I am no longer collecting data.

\section{Historical Documents}


As another secondary data collection method, I also collected historical documents from FGC partners that included FGC member surveys, meeting minutes, evaluations, timelines, logic models, promotions materials, and recruitment resources. Historical documents were collected from the formation of FGC in 2011 through the Meaning Making Meetings that followed the Gathering of Native Americans. Similar to participant observation, historical documents provided information on context, aided in documenting FGC meetings and events, and were useful in describing complex interaction within the FGC (Marshall and Rossman 2011).

\section{Data Analysis}

Prior to conducting interviews, participant observation and consultation by FGC members identified potentially significant themes for analysis. These themes were used in conjunction with themes from the literature throughout the process of thematic content analysis of FGC members' interviews and ethnographic data collected at FGC meetings and events (see Appendix B, p. 180). Themes from the literature and FGC member feedback provided the initial codes within Dedoose software. Initial codes were then used to create conceptual codes (Charmaz 2006; Strickland 1999). Focused coding for data reduction followed until theoretical sufficiency was reached (Charmaz 2006; Marshall and Rossman 2011). I also engaged in extensive memo writing and constructed code charts to facilitate thematic analysis. In this way, this data analysis strategy uses immersion in the data to develop emergent concepts and themes to ground interpretation (Marshall and Rossman 2011). 
Findings were further triangulated with data from participant observation and historical document analysis. Both participant observation and historical document analysis underwent the same data analysis procedures as for in-depth interviews. Finally, member checking was used to protect against researcher bias and to support confirmability throughout the coding and data reduction process. This included a formal Meaning Making Meeting where the findings and analysis from this case study were presented to FGC members for feedback. The Meaning Making Meeting strategically took place before the completion of thematic analysis, in order to facilitate greater FGC member voice into the analysis.

\section{CBPR Principles into this Study's Research Design}

Throughout this case study, I relied on several CBPR principles and often modeled this case study's research design after the FGC's trauma-informed research process. In this way, this case study's research design focused on: (a) trust building, (b) including FGC members crafting both research questions and research design, (c) use of culturally appropriate research methods, (d) integration of FGC member feedback into this the findings and analysis, and (e) development of long-term commitment and reciprocity.

Over the course of this research, I met with FGC members several times in order to clarify the background information within the FGC and facilitate FGC member checks of this case study's design and implementation. Several FGC partners were also consulted during the design of the in-depth interview research instrument. Additionally, the FGC incorporated a standing 15 minutes of every monthly meeting for me to discuss 
the research progress and incorporate feedback. This process helped facilitate transparency and participation in my research.

Culturally appropriate methodologies—including community participation—were used in order to facilitate participant comfort and mediate the insider-outsider effect (Brayboy and Deyhle 2000; Strickland 1999). When asked during interviews to evaluate the FGC, some FGC members offered additional unsolicited feedback on my research within the FGC. While most feedback affirmed a high level of trust from FGC members toward me as a researcher, a few FGC members offered valuable critical insight into adjustments I could make to make to strengthen my methods. This included feedback on my informed consent document as well as my use of colonized language.

As mentioned in the data analysis section, FGC member feedback was obtained through member checks as well as a formal FGC Evaluation Meaning Making Meeting. During the FGC Evaluation Meaning Making Meeting, I shared my findings and analysis with the FGC and asked FGC members for their feedback. FGC members suggested small yet profound revisions and additions to the findings and analysis. For example, FGC members suggested that "the shift" in government relationships to the Portland Native community represented an on-going process where FGC members continually worked to address and overcome the barriers of the historical trauma process. This recommendation emphasized the resilience within the FGC at a higher level than previously recognized.

At the end of the Meaning Making Meeting, FGC members were asked whether findings and analysis represented the FGC members' voices and whether the FGC approved of using this data within this thesis and subsequent reports. FGC members 
responded enthusiastically and confirmed their support, thus officially validating this case study's analysis and findings. Both during and after the FGC Evaluation Meaning Making Meeting, FGC members shared positive reports of how this case study has impacted them personally and professional. This included helping FGC members see the significance of the FGC's work, creating space to honor "the story" of the FGC, inspiring continued commitment to the FGC, and providing valuable resources for future grant writing, evaluation, partnership building, and education.

As part of my personal commitment to reciprocity in research, I tried to be as helpful as possible throughout the FGC's research and planning process. Specifically, I helped to capture data at community forums and at the GONA, contributed to the analysis of community forum data, assisted in creating an FGC brochure based on my knowledge of the FGC's process and partners, distributed fliers for FGC events, provided Elders and Natural Helpers rides to meetings, helped facilitate outreach to my husband's tribe, and acted as generally useful whenever possible. I have also committed to creating an evaluation for use of the FGC based on the findings from this case study and to work with FGC members in presenting the findings of this evaluation. The FGC will also be listed as a co-author to any future publication of this work. Since the completion of my data collection, I have continued participating within the FGC's meetings and events. This participation will continue long after the completion of this case study and into the foreseeable future.

Significance of Study 
This case study has the potential to be significant with regard to increasing knowledge the collaborations between governmental agencies and Native communities, as well as historical trauma and the effectiveness of the FGC's trauma-informed process within the Portland Native community. Specifically, the FGC's use of historical trauma within community-based participatory research and planning adds to this case study's significance by outlining an innovative model for collaborative partnerships between government agencies and Native communities. By examining the power dynamics within these relationships, this study makes valuable contributions by adding sociological insight into the literature on historical trauma and CBPR. This is significant considering a lack of attention toward Native American health issues, historical trauma, and CBPR within the discipline of sociology. For a more detailed discussion on the implications of this case study's findings, see Chapter 9: Discussion.

The context of ongoing colonization within Portland, OR and the characteristics of the Portland Native community also make this study significant. Portland, OR is a city with a growing and diverse Native community and provides an important site to understanding localized experiences of collaborative partnerships between government agencies and urban Native communities. Furthermore, this research can contribute to identifying best practices for CBPR specific to the Portland Native community, especially in research of collaborative partnerships between government agencies and Nativeserving organizations. Evidence from this report can be used to inform future initiatives, secure funding, and promote trauma-informed research and planning as a method of promoting Native American self-determination in addressing Native health issues. 


\section{Limitations}

Care should be taken when generalizing the findings from this case study to other collaborations between government agencies and other urban Native communities. The results might be transferable to other mid-sized cities in the Pacific Northwest collaborative partnerships between government agencies and other large Native communities, including Eugene, OR and three Washington cities: Tacoma, Spokane, and Seattle (Norris et al. 2012). The results might also be transferable to other collaborative partnerships between government agencies and Native-serving organizations that are engaging in trauma-informed CBPR. The significance of urbanity within Native communities also suggests that caution should be employed in transferring the results to the collaborative partnerships between government agencies and rural or reservationbased Native communities in the Northwest (Rosenthal 2000).

This study makes no claim to generalizing the perspective of the entire Portland Native community, Portland-based Native-serving organizations or government agencies within Multnomah County. It is likely that Native participants in this study are more involved in the Portland Native community than Native community members in the margins. The findings from this study suggest that the Portland Native community is not singular or homogenous. In this way, not all Portland Native communities were represented within the FGC population. For example, certain Native communities within the Portland Metro Area have not formed partnerships with the FGC, such as Portland Native community members from local Oregon tribes. Elders and men are also significantly underrepresented among FGC members. 
Additionally, while this case study's in-depth interview sample reflects strong representation of theoretically significant population characteristics and adequate representation for most other population characteristics, there exist some significant limitations. This includes underrepresentation of non-active FGC members and an overrepresentation of very active FGC members. This was due to the fact that I had greater access and established rapport with FGC members who were very active within the FGC. This may bias my results by limiting critical discussion of the FGC's traumainformed model from FGC members who were less engaged in the FGC's process. At the same time, presence of critical evaluations from FGC members included as participants in this case study and validation from FGC members during the FGC Evaluation Meaning Making Meeting help to address these concerns.

Though I am the wife of a Native man from a local tribe, I am also a white researcher attempting to conduct research within a Native community for the first time. I take very seriously my subjective experience as a white non-Native female researcher and do not assume the ability to fully represent the subjective experience of this study's participants. I also acknowledge the ways in which my commitments as a feminist researcher are influenced by euro-centric values and ways of knowing that may be distinctly different than the values and ways of knowing of Native participants within my study. Again, this caution is important considering a persistent history of white feminist researchers who have perpetuated colonial "othering" throughout the research processes (Mihesuah 2003). This came up in a few specific incidences when conducting interviews and data analysis from Native FGC members who communicated in more traditional 
indigenous perspectives. These euro-centric tendencies were flagged during member checks with Native partners and adjustments were made to better represent Native voices.

The limitations of this study also prohibit a fully formed community-based participatory evaluation. Though this study is in partnership to the FGC, ideally FGC members would be equal partners in the implementation and analysis. Greater inclusion of FGC members, especially Native FGC members throughout this research study would aid in decolonizing this case study's research methods, while establishing the highest degree of trustworthiness of results. At the same time, FGC members have expressed enthusiasm about the degree of Native engagement within this case study and approved of this case study as representing FGC member's voices.

Based on the relatively small population size of this case study, it was impossible to provide detailed demographic information throughout this study's findings while still preserving confidentiality of participants. Therefore, participants are simply described as "County partners", "Native partners", or "Natural Helpers". For the most part, County partners are non-Native and Native partners and Elders and Natural Helpers are Native American. There also exists some overlap in roles between Native partners and Elders and Natural Helpers. In both cases, revealing more nuanced information on FGC member's race and role within the FGC would likely identify my participants. Similarly, when quoting Elders and Natural Helpers all FGC members are referred to as "Natural Helpers". This is due to the small number of Elders within both this study's sample and the FGC's population. The lack of descriptive information prevents in-depth comparative analysis, however these limitation does not impair this case study's ability to address its research questions. 


\section{Conclusion}

This chapter provides details on this case study's research design and includes descriptions of the researcher's role within the FGC. Additionally, this chapter contains sections on the mixed methods used during data collection as well as the data analysis process. Reflections on the incorporation of CBPR principles into this case study's design and implementation are also outlined above. Lastly, this chapter includes a comprehensive discussion of this case study's strengths and limitations. The methodological information provided in this chapter pertains to the following findings and discussion chapters by describing how and why this case study explored the relationships between government agencies and the Portland Native community within the context of the FGC. 


\section{Chapter 5: Findings on the Historical Trauma Process}

Introduction

In the earliest days of my research with the Future Generations Collaborative (FGC), it was clear that the FGC had developed an innovative method for fostering collaboration between government agencies and Native communities. What made this organization stand out was their reliance on historical trauma as a guiding principle within partnerships development and their process of addressing Native health inequalities. Throughout my participant observation, FGC members frequently and explicitly validated the importance of historical trauma within the FGC's partnerships between government agencies and the Portland Native community. FGC members also encouraged me to include questions about historical trauma within this study's research design. In this chapter, I provide a discussion of how FGC members understand historical trauma in order to address my first research question on the government's role in creating and sustaining historical trauma within Native communities. This chapter also contributes contextual support for the upcoming discussions of how historical trauma and the FGC's trauma-informed model affect the collaborative partnerships between government agencies and the Portland Native community within the FGC.

During interviews with FGC members, participants described historical trauma in a complex and systematic way. First, FGC members highlighted the ways in which government agencies were responsible for causing historical trauma to past generations of Native Americans. Historical trauma was described as having an active role in the lives of Native Americans today through the intergenerational transmission of collective memory and cumulative disadvantage. Secondly, government agencies were also charged 
with perpetuating historical trauma systemically through a vicious cycle. The vicious cycle of historical trauma was characterized by three interlocking mechanisms, including: (a) structural inequalities within government agencies that systemically disadvantage Native Americans, (b) wide-spread and persistent enabling ideologies within government agencies that marginalize Native Americans, and (c) discriminatory actions that enact structural violence against Native communities.

While FGC members focused on the magnitude of historical trauma's impact on Native communities today, a strong theme of resilience also came through. FGC members shared a number of protective factors that help mediate the effects of both the intergenerational and systemic transmissions of historical trauma. Nevertheless, FGC members described the devastating impacts of historical trauma on Native health, social, political, and economic outcomes. FGC members also identified historical trauma as the root cause of extreme mistrust between government agencies and Native communities. In this way, the continual process of historical trauma as well as the bi-products of Native health inequalities and mistrust create enormous obstacles to the collaborative partnerships between government agencies and Native communities.

Both FGC members from the County and the Portland Native community discussed the importance of historical trauma within the relationships between government agencies and the Native communities. Throughout interviews and participant observation, most Native FGC members described their personal experiences with historical trauma, often disclosing personal or familial struggles. Native FGC members also went into greater detail describing protective factors that contributed to their healing process. Most County partners were initially exposed to the concept of historical trauma 
upon joining the FGC and developed their understanding of historical trauma through their work with the collaborative. These partners typically spoke about historical trauma as it applied to Native Americans and were less likely to share personal experiences.

Historical trauma in the relationships between government agencies and Native communities was often talked about holistically and across time and space. These discussions provide important context for understanding how macro-level phenomenon affects the local relationships between government agencies and the Portland Native community within this case study. Specific examples of historical trauma in the relationships between government agencies and Native communities in the Portland area were also identified, clarifying how the dynamic situational nature of historical trauma played out in the relationship between the Portland Native community and local County and State governmental agencies (more on these findings can be found in Chapter 6: Findings on the FGC's "Shift" to a Trauma-Informed Model). In all cases-whether centuries old or modern, local or national—understanding the government's role in creating and sustaining historical trauma is crucial for explaining relationships between government agencies and the Portland Native community within the FGC.

The Nature of Historical Trauma as a Continual Process with Intergenerational Impacts During interviews, participants recounted an extensive record of historical trauma within the relationships between government agencies and Native Americans spanning over five centuries - from initial colonization lead by Columbus in 1492 up to the modern era. Common examples included: genocide, removal from traditional lands, broken treaties, oppressive governmental programs and policies (such as the commodities 
program on reservations), removal of Native American children from homes and the coercive assimilation and abuse during the boarding school era, termination, and government-sponsored sterilization of Native American women. While many incidence of historical trauma took place decades or centuries before, many Native FGC members explained how the pains of historical trauma did not remain in the past. One Native partner said, "We struggle with it, every day. It's not something that we are going to forget." Importantly, historical trauma was not only framed as events that occurred in the lives of Native ancestors who lived generations before, but also by present generations of Native Americans. Another Natural Helper stated:

I'm a survivor of the boarding school era and was part of the horrors of boarding schools. And I still carry the physical scars of boarding schools and spiritual scars... The scars are almost gone, but they're still there and they will always be there. And so I guess I always thought, "I'm making a better life for my kids" and I never realized that they were still affected by it...because I thought it ended with me, and it didn't.

This Natural Helper's story demonstrates first-hand experience of historical trauma within her lifetime. In this way, $20^{\text {th }}$ century governmental policies have created more recent forms of historical trauma that adds to the cumulative impact of historical trauma experienced by past generations of Native ancestors. In all cases, historical trauma is described as far reaching throughout Native generations and even extends into future generations. The collective memories of historical trauma are passed down intergenerationally within Native communities, often through Native American oral traditions of story telling. This form of intergenerational transmission of historical trauma was described as having lasting consequences on the relationships between government agencies and the Portland Native community today. 
Not only did FGC members discuss historical trauma as experienced within the intergenerational collective memory of Native Americans, but also experienced through the cumulative disadvantage felt by Native Americans. A Native Partner said:

The health of my family has been impacted by historical and intergenerational trauma over the history of Native people and federal policy, assimilation efforts, all of those pieces are connected to a challenge we have as a family, my family, but the that's the same as other Indian families as well. I'm a survivor from all of that tragic history today, when for a long time I was a victim of that. I was trapped in addiction, I felt hopeless, and I put myself in unhealthy relationships...I was raised with strong supportive family members around me, but still transferring some of that intergenerational stuff through stories and interactions.

This quote demonstrates that transmission of historical trauma often happens intergenerationally within the home through stories and interactions with family members. Importantly, this Native partner remarks that her family's struggles with historical trauma mirrors the struggles of "other Indian families".

This speaks to a common theme among FGC members that Native families sometimes create unhealthy ways of coping to historical trauma, such as substance abuse and unhealthy relationships. Another Native partner explained:

So when we grow up in homes that are "dysfunctional," there are three main rules you learn, is: you don't talk, you don't trust, and you don't heal. That's in your own family and then those rules go with you everywhere in your life: in relationships, at school, at work. So you learn: don't talk, don't trust, don't heal.

Again, unhealthy or dysfunctional patterns are transmitted intergenerationally within Native families through lessons about how to relate to the world, such as "don't talk, don't trust, don't heal". These lessons are carried from the home into relationships, schools, and work places, creating more barriers to Native health and wellbeing. The intergenerational inheritance of historical trauma and unhealthy patterns and coping within Native families in turn exasperate the social inequalities faced by Native 
communities today. FGC members not only found the government absolutely found culpable for historical trauma caused by domination and oppression of Native Americans throughout the historical record, but also for the cumulative disadvantage faced by Native communities today. In this way, cumulative disadvantage creates additional strain to the relationships between government agencies and Native communities.

\section{The Systematic Perpetuation of Historical Trauma through Contemporary Traumas}

Over the course of data collection, themes emerged that clarify the systemic nature of the government's modern role maintaining historical trauma. FGC members discussed how contemporary traumas caused by governmental agencies perpetuate historical trauma within Native communities. I created a model for understanding the long-term perpetuation of historical trauma in Native communities by government agencies by pairing FGC member's descriptions of the governmental mechanisms of contemporary trauma with an interdisciplinary body of research, including adaptations of Myrdal's Vicious Cycle (1962) and Walters and Simoni's (2002) indigenous stress coping model (see Figure 4, p. 90 and Chapter 3: Literature Review).

Applying theories of historical trauma, tribal critical race theory, and Myrdal's (1962) Vicious Cycle to the data from the FGC suggests that historical trauma begins through the various colonial projects where government agencies oppress or dominate Native Americans across the historical record. FGC members described how the initial domination and oppression imposed upon Native communities during historically traumatic periods are sustained through governmental structures that reinforce the subordinated status of Native Americans. One County Partner explained, "Historical 
trauma is embedded into the organizational structures and they have to really look at how they have perpetuated some of the inequities that have happened in communities, not just in the Native community but in other communities also." Thus historical trauma feeds directly into the modern organizational structures of government. Specifically, disinvestment into Native communities, Native American exclusion from government, and governmental reliance on white-western dominated processes were common structural inequalities described by FGC members.

FGC members did not necessarily describe enabling ideologies as emerging after the formation of structural inequalities. Instead, FGC members suggested that deeply entrenched enabling ideologies within the government reflect the enabling ideologies within the broader U.S. society. One Native partner stated:

I don't believe in my lifetime, for an organization like Multnomah County or a government entity, that Multnomah County will relinquish power to a group of Native-serving organizations. It's too much of an underlying challenge around racism, oppression, systems change that I don't believe I'll see in my lifetime. Racism is engrained in our national consciousness. The way that we operate as a society nowadays, it is intuitive to the majority of our people to operate in oppressive systems.

This demonstrates that racism in broader U.S. society insulates the racism within the government. Like Myrdal's (1962) theory, this Native partner also clarifies that enabling ideologies play an important role in reinforcing the oppressive governmental structure. FGC members routinely described racism, paternalism, governmentality, and neoliberalism as enabling ideologies that perpetuate historical trauma within government agencies.

One County partner described that while working with Native communities, government agencies need "to create and sustain a culture of cultural shift in how we 
think about partnership, that's so we are not re-traumatizing or offending or engaging in micro-aggressions or just outright aggression." Therefore, governmental cultures of thought contribute to discriminatory actions against Native Americans. FGC members outlined commonplace occurrences of discrimination against Native communities within the interpersonal and institutional levels of government, including: micro-aggressions, erasing historical trauma, disinvestment, dishonesty, exclusion, marginalization, privileging white-western ways of knowing and processes, and devaluing Native culture and processes.

Based on FGC interviews, it can also be argued that discrimination deepens structural inequalities within governmental agencies' relationships with Native communities. Describing a personal experience of with the Department of Human Services, one FGC member stated:

I have felt so violated, so many times because they've treated us with so little respect and they have outright lied to us and I have proved it, you know, and have done crap around our back...And it happens all the time with Native families, when you look at the statistics. It's like I totally understand the statistics, why one quarter of Native kids in Multnomah County are in foster care.

According to this FGC member, discrimination existing on the interpersonal level between government employees and Native Americans reinforces structural inequalities within government. As demonstrated throughout this section, FGC members confirm the significance and the interlocking nature of all three mechanisms in the systemic perpetuation of historical trauma.

FGC members' experiences taken together also lend some support for the ordering of the vicious cycle as adapted for the historical trauma process. Importantly, no one FGC member described the sequence of interlocking mechanisms in this exact way. 
Therefore, the systemic perpetuation of historical trauma through the vicious cycle of structural inequalities, enabling ideologies, and discrimination should not necessarily be viewed as rigid or one-directional. At the same time, the sequence described above does provide useful insight into understanding how all of these interlocking mechanisms contribute to the government role in the systemic perpetuation of historical trauma.

To this end, I developed three examples of structural inequalities that demonstrate the historical trauma process based on FGC members' experiences and insight. Following FGC member's descriptions, these examples of structural inequalities refer to the historical trauma process' effect on the relationship between government agencies and Native communities in general. At the same time, the historical trauma process described throughout the following three descriptions of structural inequalities all contribute to our understanding of the role of historical trauma in shaping government relationships with the Portland Native community within FGC. A discussion of the historical trauma process represented in each of these structural inequalities is also relevant to discussion of the foundation of the Future Generations Collaborative in the next chapter.

\section{Government Disinvestment into Native Communities}

FGC members described termination as a federal policy that created deep historical trauma within the Native community. With this policy, the U.S. government terminated its recognition of tribal sovereignty, revoked services to tribes, and confiscated millions of acres of land that had all been protected through treaties (CurryStevens et al. 2011). FGC members suggest that modern governmental agencies continue to perpetuate the structural inequality of disinvestment into Native communities just as 
federal policy cut support to Native American tribes during the termination era. One Natural Helper explained:

There is a problem in our community that is not being addressed with current programs. And that we are really trying to figure something out that's going to be more positive for us specifically because the Native community, at least I feel like the Native community is ignored you know in favor of other minority groups...The Black community, the Asian community, and the Russian community, they all have representation in the Multnomah County area and they do have special programs for them.

This quote mirrors a common theme among FGC members who report that government agencies do not invest enough time or resources into addressing Native health, social, political, and economic concerns.

Racism and neoliberalism represent two enabling ideologies that explain governmental agencies' lack of investment into Native communities. This is particularly problematic considering the well-established role that governmental agencies have in creating the disparities that Native communities face today. Racism and neoliberalism work together to rationalize the structural inequalities of disinvestment by erasing governmental accountability to historical trauma and by blaming Native Americans for the health, social, political and economic inequalities faced in modern times. This erasure is evidenced by the many FGC member reports that government agencies and government employees are not aware of historical trauma. Alternatively, some FGC members suggested that government agencies were somewhat aware of historically traumatic incidences within government relationships with Native communities but remained ignorant to the lasting impact historical trauma has on Native communities today. Many FGC members attributed the neglect of historical trauma by governmental 
agencies to a revulsion against the concept of historical trauma and an insistence that

Native Americans "get over it". One Natural Helper disclosed:

I know for me sometimes I don't want to talk about it [historical trauma] because I don't want people to see me as extremely pitiful or to think that, "Oh she's crying over something that happened a long time ago — get over it."...And I've heard people say stuff like that, and so I'm like maybe we shouldn't talk about that because maybe this is what they're going to think. I mean the words intergenerational trauma are new but they're already getting old. You know what I'm saying? For some people they're already getting old, they're like, "Yeah, yeah, yeah. Intergenerational trauma, wah-wah-wah, stop crying, do something with yourself, get somewhere." And so it's all in a manner of perspective. I think it is important that the government does acknowledge that.

In this way, racist and neoliberal enabling ideologies erase the responsibility of the government in creating historical trauma and social inequalities within Native communities.

Racist and neoliberal enabling ideologies also help to explain the historical trauma process inherit behind the discriminatory actions taken by government agencies. One County Partner detailed:

The percentage of Native people in our community is really small. So you know, if your going to invest money into making a change, you want to see the change, but if your focused on a very small number of people in the grand scheme of things you probably won't see it and that's what I mean. There was never time and money invested in Native communities specifically, and especially not in the way that it should be done. So I mean like, everyone's in the same box. That's how they did it.

Many FGC members describe similarly how government agency decisions not to invest resources or staff into Native communities represent a highly significant form of discrimination. Another County partner stated:

And then when the Native community says, "Here's what we need." And the government says "Yeah, yeah. Thanks for that." And doesn't prioritize really clear recommendations, really clear requests for changes, not only does that build and contribute to mistrust but it contributes to a feeling of animosity, being 
ignored. And I think that in that way, if we think about a lack of prioritization as continuation of - how do I say this - the psychic genocide of Native people, the erasing of Native people, because to respond to those recommendations is to acknowledge that the community exists and to not respond to those

recommendations is a way to continue to make the community invisible.

This quote also clearly demonstrates how the erasure and disinvestment of Native communities perpetuates historical trauma today. Individual programmatic decisions to disinvest into Native communities perpetuate structural inequalities of racist and neoliberal policies and programs that marginalize Native communities.

\section{Native Exclusion from Governmental Agencies}

Various programs by the Bureau of Indian Affairs (BIA), including the Indian Health Services and boarding schools, were routinely charged with the creation of historical trauma within Native communities. Of the many ways that Bureau of Indian Affairs contributed to historical trauma was by marginalizing traditional forms of Native leadership and turning over reservation management to white government employees. This created paternalistic dynamics which many FGC members described in the contemporary unequal relationships between government agencies and Native communities. Specifically, FGC members described how the lack of Native representation within government agencies indicated an important structural inequality that perpetuates historical trauma.

Additionally, FGC members routinely described the ways in which racism and paternalism are deeply entrenched within governmental agencies. Describing her experience working with government agencies, one Native partner stated: 
These other collaboratives that I sit on it's, "Look at what we're doing." It's that, "Look at us. We're helping the poor little minority people over here." You know, "We're helping those poor little minority people over here." And it's not intended that way, but unfortunately it comes off that way.

This Native partner's description mirrors a theme that government agencies not only look down on Native communities, but also see Native communities as incapable of taking care of themselves. Similarly, one governmental partner described how racist tropes within government describe Native communities as "hard to work with", thereby revealing underlying ideas that the Native community is dysfunctional. She explained:

There's these underlying kind of ideas about dysfunction. And I think that disorder and dysfunction, looking out at communities of color and seeing disorder and dysfunction is very much, it's a function of unchecked white privilege and white culture norms. That in general white folks value order. They value linear things and processes. And to look out into community and to see what might appear to be chaos or dysfunction, that feeds into that loop of mistrust or unwillingness to be a part of solution making.

Both quotes exemplify how racist and western-ethnocentric values also prompt government agencies to rationalize disinvestment into "solution making" and other forms of collaboration with Native communities. If Native communities are looked down upon with these racist and paternalistic values, then the modern structural inequality-lack of Native representation within government agencies-is rationalized.

Governmental values and beliefs that Native communities cannot take care of themselves and are dysfunctional contribute to the discriminatory actions in the form of racist micro-aggressions. FGC partners frequently described how these racist microaggressions often occurred within collaborative settings between government agencies and Native organizations. This invariably creates an exclusionary environment toward Native voices at the table. Unfortunately, racism and paternalism also frequently 
influence the decisions of who is invited to the table in the first place. Many FGC members reported various exclusionary practices that contribute to an inordinate lack of Native representation within government agencies.

FGC members described governmental discriminatory exclusion of Native Americans in various forms. In many cases, FGC members suggest that governmental exclusion of Native Americans is based on a lack of investment into making meetings and events accessible to the Native community. Neglecting to provide childcare, food, and transportation were all described as exclusionary actions that contribute to the lack of Native representation at governmental meetings and events. FGC partners also described a lack of investment into partnership outreach with Native organizations. For example, one FGC member described an incident where governmental agencies wrote a local Native organization into their grant without asking them. Thus government agencies are able to avoid inclusion of Native communities and organizations through various methods of discriminatory exclusion.

FGC members also described how Native exclusion exists within the governmental agencies themselves. For example, many FGC members noted the extremely small number of Native employees working within local government agencies in Portland, OR. Further, FGC members described how the few Native employees were often positioned as token minorities within the government agencies they served. One County partner stated, "Really it takes all. It takes all the system. They've got to quit segregating this work, like, 'That's the Indian problem. Our Indian employee will take care of it.' Many agencies do that." Shifting the responsibility of addressing all Native issues onto one Native employee is a discriminatory action that allows government 
agencies to get away with only the most perfunctory level of Native inclusion. Thus the vicious cycle demonstrates that government agencies perpetuate the historical trauma of oppression by the Bureau of Indian Affairs while also creating contemporary traumas by systematically reinforcing Native exclusion.

\section{White-Western Domination within Government Agencies}

FGC members continually held the boarding schools responsible for Native American cultural genocide. Boarding schools created immense historical trauma through the forced assimilation and violent prohibition of Native traditional languages, practices, and beliefs. Bureaucratic structures within modern government agencies were also frequently described as white-western dominated systems that are guilty of imposing hierarchical and culturally inappropriate programs and policies. One Native partner stated:

It's just it's so insane and so archaic the way most systems are, whether they are Native or non-Native. And I'm sorry but in some of the tribes they are so straight off the boarding school model that it's worse than any of the State. It's like 1950 in terms of hierarchical protocols and procedures that really again sabotage the unity of people because that's not the way the culture works. Actually, I think it sabotages the unity of any people to have hierarchy. I mean it basically is the model of the oppressor for a reason.

In this way, the structural use of hierarchical protocols and procedures work against Native culture and sabotages unity, thus continuing the subordination of Native Americans by governmental agencies. The boarding school model is also described as responsible for creating historical trauma in Native communities that has been translated into the structure of some tribal governments. 
Racism, paternalism, and governmentality were frequently described enabling ideologies that contribute to government agencies' strong propensity for privileging white-western ways of knowing. For example, FGC members describe how linear outcome-oriented philosophies dominate government agencies in ways that marginalize the holistic and relational perspectives common in many Native cultures. One Native partner remarked:

[The circular model] it is not a less efficient model. I guess that's a hugely important message to me, because what I feel like when I try to explain it sometimes is patronized in terms of "Well, yes that's beautiful. That's very sweet. Okay when you're done we'll translate that into a working model, a linear model." And I don't....and I think no one means any harm by that, but what it tells me is no you aren't getting my point. There are these fundamental differences. We may have a common goal, but there are these fundamental different processes. And particularly if we are a process-oriented people we are going to have a fundamentally different process because we're looking at it in all direction so to speak.

Government agencies' lack of understanding for Native ways of knowing along with Native processes is confirmed by this Native partner's experience. The paternalistic way governmental agencies devalue holistic processes as "less efficient" also rationalize the systemic inequalities of government reliance on white-western dominated processes.

Furthermore, racism, paternalism, and governmentality are made manifest in two forms of discrimination: patronizing micro-aggressions and the marginalization of Native perspectives and processes through forced conformity to white-western standards. FGC members continually described experiencing governmentality and "my way or the highway" type thinking through the discriminatory actions of governmental employees.

One Native partner reflected the common theme that government agencies are not listening in partnership meetings with Native communities. She said: 
Because a lot of times I'll walk into a meeting and I'll know they're not going to care, and I'll just say, "Yada yada, blah blah. I think blah blah." Yeah, you know? And it's really just like frosting a cake and it really is not-you know it's not going anywhere, you know it's not going to be very effective and you just know you're not being heard. You're just in that room to represent. And that really kind of sucks.

This Native partner's reflection illustrates that even when Native communities have a seat at the table, government agencies are not truly invested into partnerships with Native communities. FGC partners frequently described the bureaucratic way that government meetings are conducted as disrespectful and culturally inappropriate. Specifically, reliance on linear, hierarchical, and outcome-oriented processes were described as discriminatory practices by governmental agencies that alienate Native partners. FGC members also explained how rigid and rushed governmental processes similarly create a culturally unsafe environment for Native communities.

FGC members said that the white-western dominated language during meetings discriminates against Native communities by forcing Native partners to constantly change their language to the language of the government, or "code switch". One County partner stated, "It shouldn't be the Native community always having to code switch. The government agencies should also need to code switch. Because it's always upon the minority community or the minority employee to be the one who has to code switch." Forcing Native Americans to code switch during meetings is problematic because it reinforces unequal partnership between government agencies and Native communities. Additionally, the exclusive use of white-western language also marginalizes Native epistemologies. One Native partner explained:

While working on this one committee, someone said, "Okay you tell us and then we'll translate it into our language." Then I said, “Then you've missed my whole 
point. Why would you translate it into your language? If your serving Native people why don't your translate it into our language?" And a well-meaning person, but so often the thing is, "Okay, we get it, we get it, we get it. We understand. Okay now we'll put it into best practice or clinease language." And no, it really needs to be in the first place we've already removed it at least once from whatever traditional language these concepts are coming from. So we've already profaned it but let's not try to put it into a linear language because the whole point is that it's not.

This quote demonstrates that white-western dominated language alienates Native partners during planning meetings with government agencies. White-western dominated language also alienates Native communities who are the intended recipient of governmental services. As follows, Native communities were also described as profoundly affected by one-size-fits-all programing that ignore the cultural differences between Native communities and broader society. Thus, the creation of one-size-fits-all programing represents another kind of governmental discrimination that marginalizes Native Americans and contributes to ineffective programs.

Throughout FGC members' descriptions of partnerships between government agencies and Native communities another theme emerged that speaks to a high degree of dishonesty among government agencies. A Native partner said:

In my own experience, they never are telling the truth. It's always something else. They say they understand - that they hear your concerns and that they understand and then they'll give you the lip service. They'll be like, "Oh yeah, we totally get that. We want to work with you and we're going to make that happen," but then you'll turn around and you'll see these actions that don't reflect what they were saying and that they weren't telling the truth...You've always got to be on your toes and be ready to call them out on that.

This illustrates the common theme that government agencies will often pay "lip service" to Native communities without actually "walking the talk". FGC members also commonly describe governmental meetings with a "lack of transparency" or "a lot of 
fluff and not any significant output" as dishonest and therefore represent discriminatory actions by government agencies. Governmental dishonesty also came up in the research setting where often government agencies come into Native communities, collect data, and leave without delivering any of the promised benefit. In other cases, government was cited as using research against Native communities.

This plethora of discriminatory behaviors taken by governmental agencies serves to perpetuate the structural inequalities of white-western dominated systems by creating unequal partnerships between government agencies and Native communities.

Furthermore, the discriminatory behaviors of government agencies within the planning also perpetuate to the structural inequalities by contributing to ineffective and culturally unsafe programs and policies. In both ways, discrimination in the collaborative setting plays an important role in the continuation of historical trauma over the long term.

\section{The Role of Native Protective Factors in Promoting Resilience}

Throughout FGC member interviews a strong theme of resilience came through, especially among Native FGC members. Resilience was discussed in terms of Native protective factors that contribute to "healing from historical trauma". Importantly, most FGC members described healing as an on-going process in which the cumulative influence of Native protective factors create relief from the ongoing process of historical trauma. As a result of the healing process, Native protective factors contribute to resilience in terms of Native health, social, political, and economic outcomes.

The persistence of Native health, social, political, and economic inequalities suggests that protective factors within Native communities are not yet at the point where 
they can completely reconcile the impacts of the historical trauma. One Native partner stated, "It didn't happen overnight and it's certainly not going to be fixed overnight." Nevertheless, the degree to which healing was discussed reflects how highly valued a strengths-based approach is within the FGC. A Native partner stated:

If you do an asset inventory, if you look at any of these needs from a strengths based perspective, which I think is mandatory that you do, is that you realize that you have within any of our communities - not matter what are its challenges - we have a tremendous set of assets and resources.

Integrating Native community "assets and resources" into our understanding of the challenges that Native communities face today is of great importance. Therefore, protective factors are included within this study's theoretical model (see Figure 4, p. 90). Just as most Native FGC members shared from their lived experiences of historical trauma, they were also able to share from their personal anecdotes about how protective factors contribute to Native resilience. The following section details how individual, family, community, and cultural protective factors function as part of the healing process. Many FGC members described healing as a process that begins on the individual level. A Native partner described how personal awareness was the first step in empowerment, stating:

That awareness part, when we start to get rid of some of the stuff that holds us back, you know that's what I call healing, right? To become whole, then we can be empowered, and when we're empowered then really the sky is the limit. When we're empowered we can start to want something better for ourselves and then that kind of grows and we want something better for our families and then that grows and we want something more for our community.

This quote explains that becoming empowered acts as an individual protective factor by enabling Native Americans to "want something more" for themselves, their families, and their communities. Similarly, Native FGC members frequently identified as "survivors" 
of historical trauma, thus highlighting their own individual resistance and ability to

overcome extreme adversity. One Natural Helper stated:

I, myself am a survivor of historical trauma. I'm living proof that we can survive...That's my job in the Native American community and I take that job on willingly. People don't need to ask me to. People don't need to appoint me as that person. My job is to stand up when nobody else can and my job is to be there to help when nobody else can. And I see a lot of the Elders in the community, they're worried because they're survivors of historical trauma. I listened to one Elder say, "You know what, I look around at all you young men, this is what I do as part of the community, and none of you recognize it no more." ...And so I view that as I'm here, show me, because we have suffered enough.

According to this Natural Helper, the survivor identity acts as a protective factor by bolstering personal strength and a willingness to stand up to injustice.

Another common theme among Native FGC members was that learning about historical trauma helps to "break the cycles" of unhealthy coping mechanisms. Native FGC members often described learning about historical trauma as an important part of the individual healing journey. One Native partner stated:

In my recovery experience as I started to heal and learned about intergenerational trauma/historical trauma, that opened-I mean, that brought daylight into my world. I was like, "Holy Moly, there's a reason that I'm experiencing this really deep sadness, when from outside appearances my life looked normal and I had every reason to celebrate rather than be sad." And I attribute that to the historical trauma components.

Being able to pinpoint historical trauma as the "root cause" of many FGC members" personal struggles also strengthened healing. Furthermore, this quote also highlights the importance of sobriety as an individual protective factor that helps break the cycles of addiction within Native communities. Taken together, FGC members descriptions help clarify how individual healing was important the healing of Native communities as a whole. A Native partner explained, "Creating more healthy community members is only 
going to increase the health of the community and the welfare of the community. And I

think that is something that I have a real personal plus with: breaking cycles". In this way, individual protective factors are an important component of the Native healing process.

FGC members commonly referred to Native families as the next important site for Native healing from historical trauma. Native families provide several protective factors that promote Native resilience. One Native partner elaborated on the importance of family, explaining:

I don't come from a perfect family. I do come from a family that has always hung very close together over generations, that knows its story, in it's mix, the choices that were made and why we've been here for generations now. But not a perfect family by any matter of means. So within the context of what I've learned as a child, as various extended family members struggled equally, then I saw that because I chose to work with children. It was immediately known to me that you don't work with children without working with parents. You don't work with parents without working with grandparents and it is a multigenerational circle. And that of course is what my grandmother has taught me and showed me every day because I grew up in a multigenerational household. There were three, actually there were four generations while my great grandmother was alive. So that gives, even with all of its up and downs and ins and outs, it gives a certain anchor and balance to a life.

This Native partner's story specifically details a wide array of family protective factors, including: sharing stories, cultivating identity, providing connection, wisdom, and traditional knowledge, as well as facilitating balance and shared responsibility for child rearing. Additionally, this quote demonstrates the common theme that multiple generations of family members are needed for the promotion of healing in Native communities. FGC members also described the role of their Native family members in promoting Native resilience and healing through their resistance to colonialism and their maintenance of cultural traditions. A Natural Helper stated: 
My grandma, my mom's mom, she always told us stories and even though my mom didn't really live a Native culturally appropriate life-style or whatever, my grandma always smudged us, told us stories, sang us songs, made us fry-bread, stuff like that. She made sure we knew what was up, you know. She would braid our hair and show us pictures of dresses and all the stuff my mom wasn't teaching us. So that's kind of little bit of background about me. When I was nine I moved to the reservation and I got a full experience of the rez life. And my dad is in is a secret medicine society of my people, so he is a healer, he was a healer, he passed away last year. He exposed me to a lot of spirituality, and tradition, a lot of ceremonies. And I learned a lot really fast, really quickly.

This demonstrates that the multigenerational family is crucial for the providing the Native protective factors of spirituality, ceremony, tradition, and healing. Therefore, Native families provide an important site where healing takes place together.

Native communities also enable Native Americans to engage in healing at the group level. Participation in Native communities was often described in terms of participation within one's own tribal community as well as participation in Portland Native communities. Native FGC members frequently described their relationship to their reservation and described how the reservation provided protective factors of access to tribal-specific culture, identity, family, and services as well as access to tribal elders. In both the reservation and urban settings, Elders were described as critical members of the Native communities contributing heavily to the preservation of knowledge, culture, and wisdom. Since most Native FGC members do not come from local tribes, Portland Native communities provide essential protective factors that might otherwise be out of reach. Most Native FGC members are deeply involved in the Portland Native community and participate regularly in community events, programs, and services. One Natural Helper described:

I'm really involved with all of this stuff, and I love it because it's really making changes in the community. There's so many things going on in our Portland 
Indian community — and I get to be a part of it, and not-I wouldn't say the leader of it, but I get to be a part of it. And so I really enjoy that.

Native communities provide protective factors of opportunities for involvement, enjoyment and wellbeing. Moreover, participation in Native communities promote selfesteem by provide Native Americans with opportunities for being of service and "making changes in the community".

Similarly, Native organizations, such as the Native American Youth and Family Center (NAYA) and the Native American Rehabilitation Association (NARA) were frequently described as important pillars in Portland Native community in terms of access to culture, community, and resources. Another Native partner explained:

And that's what brought me actually to the Portland area, because there's a strong Indian recovery community here, a Native American sobriety movement if you will. And coming - and I did some time at the NARA residential treatment center, I'm a NARA alumni-that experience for me was really like coming home, I was reintroduced to some old cultural ways that I was familiar with. I really was given space to be removed from the domestic violence component and reflect and learn who I was as an individual and getting to know myself, that experience allowed me to bring my children back into my circle and reunify my family, and really life has been blessed ever since then.

This quote clearly demonstrates the important role Native organizations have in providing protective factors to promote resilience in terms of Native health and social outcomes. Importantly, many FGC members describe the NARA residential treatment center as a draw to Portland. In this quote, NARA provides valuable resources that contribute to sobriety, removal from abusive situations, family reunification, selfknowledge, and advocacy. Since many Native FGC members work for Native organizations, Native organizations also offer employment, economic opportunities, and a sense of purpose for Native Americans. According to FGC members, these are just 
some of the many protective factors available to Native Americans through Native organizations.

While Native culture is integrated into FGC members' descriptions of the Native individuals, families, and communities protective factors, it also represented a protective factor in its own right by tying Native individuals, families and communities together through ceremony and tradition. Native cultures were also frequently described as connecting Native Americans to the land. Finally, culture was described as changing over time in ways that strengthen the Native community. For example, one Native partner told a story of how the American Indian movement contributed to a Native culture of sobriety by changing community norms. She stated:

But when the norms start changing then there's going to be more people that lift them up. They're surrounded by people that are lifting them up, right? That contributes to their self-empowerment. So an example of norms changing, in the 1970's, during the civil rights movements, our people were like, "Well, what about us? What about our rights?" And so the American Indian movement and different things like that sprung up and our people said, "Well if we're going to be taken serious, we need to quit drinking." So in the 1970's was this whole sobriety movement that swept across Indian Country and our norms as a whole started changing. For example, early in the 1970's and even into the 1980's you would go to a pow wow, as an example, and you would see drunk people all over. Drunk people staggering around, drunk people passed out, drunk people sitting in the bleachers, and that was the norm. Nobody did anything about it. I guess sometimes one was escorted out or something like that. But now the norms have changed and you never see that. You never see that, okay...And so those are like big examples of norms changing, but that's what I'm talking about of community norms.

Shifting community norms provides Native cultural protective factors that enable Native Americans heal from the impacts of historical trauma and strengthen Native resistance to oppressive systems. 
In this way, the Native healing process not only contributes to healing from historical trauma and resilient Native outcomes, but also to empowering Native communities to resist the perpetuation of historical trauma by governmental agencies. One Native partner also suggested that Native resilience reduces the severity Native mistrust of government agencies by helping to reduce "reactionary" responses to government agencies. She said:

I mean that's part of our own healing as well. You know what I mean? Is not always looking at the government as out to get us, or something like that. And even our own personal healing allows us to change our attitudes about that to. That we don't need to hold on to that anger, you know?

In both ways, Native protective factors play an indirect yet significant role in shaping between government agencies and Native communities.

\section{The Potential Role of Governmental Protective Factors in Promoting Resilience}

FGC members went into great detail describing not only the government's role in creating initial historical trauma in Native communities, but also in perpetuating historical trauma over the long term. One County Partner stated, "If agencies continue to perpetuate traumas to families and traumas to communities, that cycle is never going to end." This reveals the potential role of government agencies in helping to stop the perpetuation of historical trauma. In fact, most FGC members describe government agencies as having both a moral obligation and unique responsibility in addressing historical trauma in Native communities.

Many FGC members made clear the importance of government agencies understanding their continued role in the historical trauma process. This understanding 
was described as a crucial starting place for governmental accountability. A Natural

Helper shared this concern:

I think they [the government] should acknowledge what the truth is. They should acknowledge the destruction and the chaos and the murder and the rape and the pillaging that were imposed upon or people, and they should acknowledge it and as an entity they should say, "This was not okay, and we know that everything changed because of it, and we know that things are still changing because of it". And ultimately, you know the end result for us is death-death and destruction: death of our people, death of our culture, death of our language, death of our everything.

Thus for many FGC members, government agencies ability to acknowledge their active role in the perpetuation of historical trauma has impacts on Native community relationships with governmental agencies. For many FGC members, this understanding of historical trauma was also absolutely essential for a holistic understanding the health, social, political, and economic inequalities experienced by Native Americans. Some FGC members suggested that until government agencies were able to firmly grasp their role in creating and sustaining historical trauma, government services and programs were likely to have little effectiveness in Native communities.

Understanding historical trauma was described as necessary for lessoning the effect of historical trauma. One Native partner said:

How you understand the historical trauma and how you make-I don't think you're ever going to make it go away, but how you make some sort of adjustment and reparation and how you make it better today than it was yesterday? That's a real big thing, if you work at it you can make it different. But you have to make a commitment to make it different and you have to really step outside the box to make it different if you are from a governmental agency.

Echoing this quote, nearly all Native FGC members stated that historical trauma would never be forgotten. Yet most FGC members agreed that the effects of historical trauma could be alleviated on the basis of understanding by governmental agencies, followed by 
"adjusting" to stop creating contemporary traumas, and "repairing" to address historical traumas. As this Native partner described, the call of government agencies to adjust and repair is no small task and takes a high level of commitment and willingness to "step outside of the box". This once again speaks to the degree that historical trauma is engrained into contemporary governmental systems and the enormity of confronting the systemic perpetuation.

Finally, some FGC members described how government agencies' understanding historical trauma was crucial for cultivating healing within Native communities. A Native partner confirmed:

I truly believe that one of the key answers to our people moving forward from the cycles of poverty, the cycles of negativity, the cycles of chaos, the cycles of dependency, the cycles of substance abuse and all that, is healing...So, historical trauma then and healing and the relationship to the government and all that kind of stuff, it's all inter-related.

This suggests that government agencies have a role in supporting healing from historical trauma in Native communities. At present, FGC members' descriptions of the level of mistrust between government agencies and Native communities as so high that government agencies will likely have to support Native resiliency indirectly through the support of Native protective factors.

At the same time, FGC members suggest that the government can potentially make historical trauma "a little better than it was yesterday" by (a) acknowledging governments role in creating and perpetuating the historical trauma in Native communities, (b) making adjustments as necessary to stop the vicious cycle of historical trauma, and (c) supporting Native protective factors. Through all this work, FGC members suggest that government agencies may have impact on the level of trust in 
government relationships with Native communities as well as second-hand impacts on Native health, social, political, and economic outcomes. Nearly all FGC members made suggestions about what government agencies could do to reduce the impacts of historical trauma rather than what government agencies have done. This reflects a common theme that leading up to the creation of the FGC, FGC members had no experience with government agencies working to address their role in historical trauma.

\section{Impacts of the Historical Trauma Process on Native Community}

Across the board, FGC members agreed that historical trauma has profound impacts on inequalities experienced by Native communities today. Specifically, FGC members described the impacts of historical trauma on Native American health, economic, political, and social outcomes. One Natural Helper stated:

The government, they are mostly responsible for Native Americans and a lot of the struggles that we have been involved in. You know, relocation, our children being separated from us, experimental treatments that we had to be put through when we did seek health care, having our diets changed because of commodities that they had decided we needed. So they did have a huge part in a lot of the struggles that we have now.

This Natural Helper's connects relocation, boarding schools, and the commodities food program to drastic changes in Native American diets. Other FGC members made this same connection explaining how the changes in the diet explain the high rates of diabetes in Native communities today. Similarly, experimental treatments initiated by the government were linked to barriers to receiving health care services for Native Americans. These are just two of the many examples of how historical trauma is directly 
tied to Native health outcomes. Other common themes included historical trauma's impacts on Native American mental health, addiction rates, and reproductive health.

FGC members made similar connections between historical trauma and the economic inequalities experienced by Native Americans today. A Native partner stated, "We live in poverty because of the impacts of the boarding school era. Termination of tribes was to remove resources that provided economic development for our tribes. We were impacted by those resources being stripped." What is revealed here is that boarding schools, termination, and extraction of resources from tribal lands directly tie into the poverty rates experienced in modern Native communities. As described previously throughout this chapter, many FGC members also spoke to the political inequalities experienced by Native Americans through descriptions of Native exclusion from government agencies.

Another Native partner described how the removal of Native children from their homes during the boarding school era contributed to an array of social problems, including: loss of culture, loss of parent-child bonds, and loss of connection to the land, as well as contributing to high rates of substance abuse. She explained:

And because the trauma that occurs to the child as well as the family, because I'm pretty sure I know what I would do if every child was removed from me at age five, but that translates into generational trauma... It wasn't just - we speak of it, "It took the language, it took the culture." Those were core pieces, but it took the bond. It took the bond from parent to child, it took the bond from land to child, and it took it from what we know are really important years... That's what residential schools did. They took every reason to trust away at a really critical time of development and that's why where I say, because other cultures lost everything in terms of land, lost everything in terms of life too, I mean there have been many other genocides, but it was this taking of the children that really took the hearts. And it didn't just put them on the ground it buried them in the ground and if any air touching then just please give me medication to dull that pain, is all that I can imagine. 
Many Native FGC members shared deep insight into the impact of residential schools on Native social problems, including: loss of culture, language, family, and land as well as contributing to high rates of depression and addiction. This quote also represents the continually cited theme that government are responsible for both creating the initial incidence of historical trauma as well as the cumulative impacts on Native health, economic, political, and social outcomes. Native FGC members also described personal hardships caused by historical trauma and how these lived experiences contributed to mistrust of government agencies. Therefore, the lived experiences of Native health, social, political, and economic inequalities influence the relationship between government agencies and Native communities in profound ways.

\section{Impacts on Government Relationships with Native Communities}

Throughout interviews with FGC members, the significance of Native American mistrust of governmental agencies cannot be overstated. All FGC members reported vast amounts of Native American mistrust of governmental agencies as a result of historical trauma. One Native partner described whether or not the government could be trusted, stating, "We've had no reason to trust them because we've only been given reason to not trust them." This quote demonstrates the powerful influence historical trauma has on the relationship between government agencies and Native communities.

While most FGC members acknowledged Native community mistrust of the government at large, Native FGC members also spoke to their personal levels of government mistrust to varying degrees. At one end of the spectrum, some Native FGC 
members said that their trust for government agencies and government employees was nonexistent. One Native partner said, “There isn't trust. I don't think that trust exists. I think that if we're going to be smart about anything we wouldn't trust anybody that works for the government... And I think that the track record speaks for itself, that they aren't to be trusted." On the other hand, some FGC members highlighted the persistence of Native mistrust while also acknowledging the difference between contemporary government agencies and government agencies in the past. Another Native FGC member stated:

I also feel like the government then is not the same one as it is now. Even though there is a lot of reasons to question our government, today I feel like local governments, local agencies are more open to helping improve the lives of Native Americans because it's not the government overall that is bad, it's just the people that had initiated and okayed these things in the government really made it look bad for all of them...I think we need to start seeing our government employees as individuals and not part of big entity.

The majority of Native FGC members reported high personal levels of mistrust without writing off the potential to build trust in the future.

Throughout FGC members discussions of mistrust a clear pattern emerged. The government was responsible for creating extremely severe historical trauma in Native communities, which resulted in a powerful mistrust of government agencies by Native communities. Historical trauma and mistrust then act in tandem to create large barriers between government agencies and Native communities. One Native partner stated, "I think historical trauma is like the number one thing that we struggle with. I think it's the number one thing we struggle with as individuals and I think it's the number one thing we struggle with in terms of community partners." FGC members also describe how historical trauma and the legacy of mistrust creates a reluctance of Native organizations 
to partner with government agencies. Likewise, FGC members explain that Native Americans sometimes avoid government services on the basis of historical trauma and mistrust. Therefore, mistrust reinforces the Native health, social, political, and economic inequalities experienced by reducing Native communities access to governmental resources. These three examples illustrate the profound affect mistrust has on the relationships between government agencies and Native communities.

\section{Conclusion}

Throughout this chapter, FGC members provide critical insight into government agencies' role in creating and sustaining historical trauma within Native communities. In doing so, FGC members describe the historical trauma process in complex and systematic ways. By combining FGC member's evaluations with the body of literature on historical trauma, tribal critical race theory, and Myrdal's (1962) vicious cycle, I create a model for understanding the historical trauma process in Native communities (see Figure 4, p. 90). The findings from this chapter lay the groundwork for the subsequent chapters' findings on how historical trauma affects the relationships between government agencies and the Portland Native community within the context of the FGC. Moreover, this chapter provides essential contextual information for the upcoming findings on how the FGC's trauma-informed process affects the relationships between government agencies and the Portland Native community within the context of the FGC. 


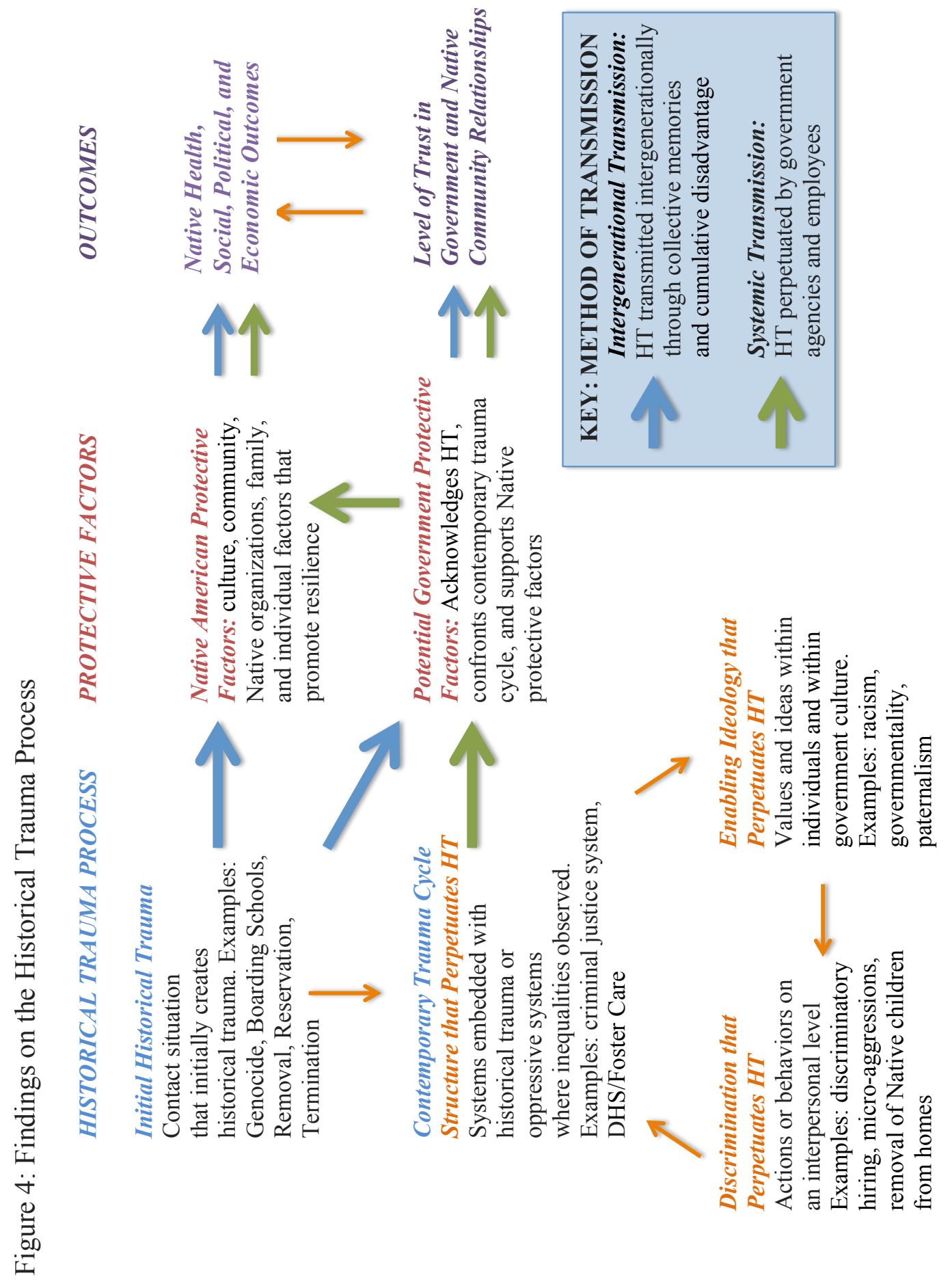




\section{Chapter 6: Findings on the FGC's "Shift" to a Trauma-Informed Model}

\section{Introduction}

While the historical trauma process outlined in the previous chapter describes the role of historical trauma in the relationships between government agencies and Native communities generally, it also provides specific context to government relationships with the Portland Native community within the Future Generations Collaborative (FGC). In fact, FGC partners outline the government's role in the systemic transmission of historical trauma through the structural inequalities of disinvestment into Native communities, Native exclusion, and white-western dominated processes through their descriptions of the Multnomah County Health Department's initial approach in creating a Native maternal child health initiative. FGC partners' accounts also demonstrate that structural inequalities were both reflexive and reinforcing of the enabling ideologies and discrimination used in the systemic perpetuation of historical trauma. At the same time, FGC partner's descriptions highlight the important ways in which structural inequalities were challenged from the onset of the FGC's formation, thus making way for a shift in the Health Department's approach to partnering with Native communities in Portland, OR.

Several contributing factors aided to what FGC members commonly refer to as "the shift" in relationships between government agencies and the Portland Native community. First, Native partners demonstrated extraordinary resilience and resistance to the systemic transmission of historical trauma by County partners from the Health Department. Second, County partners were willing to (a) acknowledge their departments' roles in creating and sustaining historical trauma within the Portland Native community, 
(b) commit to making adjustments as necessary to stop the vicious cycle of historical trauma, and (c) support Native protective factors. These factors contributed to the shift in the goals, organizational roles, and processes of the County partners. Ultimately, this shift lead to the formation of the Future Generations Collaborative and the creation of the first trauma-informed community-based participatory research and planning process between government agencies and Native communities.

The Systemic Perpetuation of Historical Trauma through the County's Initial Outreach

Before the FGC was established, an epidemiologist from the Multnomah County Health Department noticed that there were no maternal child health programs that used cultural competency specific to Native Americans. A County partner explained:

Her other observation was that we had other culturally specific programs, particularly health birth initiatives, which is a program that is an individual and community level intervention that specifically works with the African American community in addressing low infant birth weight and infant mortality. And it was concerning to her that actually American Indian/Alaskan Native rates for those indicators aren't that much different. In fact, American Indian/Alaskan Natives have higher rates of infant mortality than African Americans, but as a department we weren't addressing it in a culturally specific way. And she was kind of wanting us to ask, "Why is that?" and "What can we do about it?"

The structural inequality outlined in this quote mirrors the theme of Native disinvestment outlined in the in the section on government disinvestment into Native communities in the previous chapter. Furthermore, the Health Department's lack of prioritization of Native communities represents both racist and neoliberal enabling ideologies. These enabling ideologies contribute to the precedence of discriminatory actions in which the health needs of Native Americans are neglected in Portland, OR. Importantly, this quote also details how the County members' recognition of a structural inequality within the 
Health Department provided the initial motivation for a Native maternal child health initiative.

Soon after, the County epidemiologist began assembling a team of government stakeholders to apply for a technical assistance grant for building capacity around the issue of substance-exposed pregnancies in the Portland Native community. Unfortunately, the initial team did not include any Native partners from within or outside government. One County partner detailed:

I have to say that the first time I walked into the room, it was a lot of Health Department employees, State Health Department employees. There was really no outside agencies except for the State, which came from Maternal and Child Welfare also. And so I looked around the room and there was no Natives in the room. And I was like, "Well you got the wrong people at the table." You know, not necessarily that they were all wrong, just that they didn't have the Native partners, and they were struggling to get the Native partners.

The County partner's assessment within this quote parallels the theme of Native exclusion from government agencies described in the previous chapter. This again reflects racist and paternalistic enabling ideologies that assume that government agencies know what is best for Native communities. This explains the Health Department's discriminatory actions of creating a stakeholders group on a Native health issue without any Native representation. However, the County member's concerns about the lack of Native representation were heard, leading to outreach to some of the local Portland Native organizations.

Before the internal committee made outreach efforts to the Portland Native community, they first established a plan to invite Native stakeholders to provide input on a culturally specific screening, brief, intervention and referral to treatment (SBIRT) intervention for Native Americans. SBIRT is a national evidence-based practice that acts 
a primary care intervention (Substance Abuse and Mental Health Administration 2014).

Initially, two Portland-based Native organizations agreed to take part in the project. A

County partner describes the initial partnership meetings, stating:

In the beginning it was County partners that facilitated the meeting, like came up with this meeting structure and stuff, and we had a different approach first. We were like, "let's gather all of the data, all of the facts, and show it to people to convince them, and to compel them, and to spur them into action because data moves people. The evidence is there." But obviously that didn't work because everyone has seen this information. It's the same story told over and over again.

This quote reveals the initial top-down approach used by the internal committee during the FGC's foundation. The Health Department's initial approach aligns with FGC descriptions of white-western dominated processes within governmental systems as outline in the previous chapter. Again, racist and paternalistic enabling ideologies that value linear, hierarchical, and outcome-oriented processes help to explain the Health Department's discriminatory actions in their culturally inappropriate approach.

The Multnomah County Health Department's approach received harsh scrutiny from one Native organization. The fact that County workers were well intentioned and did not understand the historical legacy of their miss steps did not necessarily lesson the degree of mistrust that arose from the partnering Native organizations. A County partner recalled:

When we approached this one Native organization, they said, "You can't just come up here with out any relationship, ask us to sign on to this multi-year probably project that doesn't have one red cent to its name, it's just not okay because we don't have any history."

Reflected here is a deep skepticism of Native partners based on the structural inequality of government disinvestment into Native communities and the discriminatory action of asking a Native organization to sign on to a project with no funding. Similarly, this 
Native partner's statement that "we don't have any history" illustrates the precedence Native exclusion from partnerships with the Health Department. Lastly, this quote demonstrates that the Health Department had already designed multi-year project before approaching the Native organization for partnership. This reflects a top-down whitewestern process that is culturally inappropriate and clearly creates mistrust among Native partners.

Unbeknownst to the County partners, the department they were representing, the Multnomah County Health Department, had also engaged in another discriminatory behavior against the Portland Native community: neglecting the historical trauma between their department and the Portland Native community. FGC members described how many Portland Native community members view the Multnomah County Health Department as a symbol a recent historical traumatic period of forced sterilizations.

According to the Communities of Color Coalition:

During the 1960s and 1970s, involuntary sterilization of Native American women was rampant. Legalized in the early 1900s and enacted until 1983, the State of Oregon permitted involuntary sterilization, using it often as a condition of release from state institutions... Our County-run health clinics were one of the institutions that took part in these sterilizations...Sterilization included coercion of women of child-bearing age along with forced sterilization of women in state institutions. The numbers were huge, ranging between $25 \%$ and $40 \%$ of Native women of childbearing age (Curry-Stevens et al. 2011, p. 65).

FGC members similarly described how the coercive sterilization by the Multnomah County Health Department had deep impacts on the levels of Native American mistrust of government agencies and deepen the health inequalities experienced by the Portland Native community.

A common theme among FGC members was the ways in which historical trauma 
from the Multnomah County Health Department's use of coercive sterilization contributed to the high rates of substance-exposed pregnancies within the Portland Native community. This historical trauma was transmitted intergenerationally through collective memory and cumulative disadvantage. Additionally, FGC members described how historical trauma from sterilization is compounded and transmitted systemically through the structural inequalities, enabling ideologies and discrimination within governmental agencies. Both of these processes lead to elevated rates of substance abuse and other high-risk behaviors that increase the risk for substance-exposed pregnancies within the Portland Native community. Native mistrust caused by the historical trauma of sterilizations also reinforces the mistrust of social service agencies providing reproductive care. In this way, historical trauma from sterilization again leads to increased rates of substance-exposed pregnancies by creating barriers reproductive health services in the Portland Native community.

The Health Department's role in contributing to Native FASD rates lead Native partners to criticize the initial committees request for funding assistance from Native agencies for an FASD initiative. A County partner recalls feedback from a Native organization:

It's frustrating for us that we did not create these problems, we did not create the structural violence and the inequities that are leaving disproportionate levels of FASD in our communities and yet government agencies come to us and ask us to put staff time into writing grants and finding funds. It's like no you need to find the funds. You need to do the work.

Again the government's lack of accountability for their role in the systemic perpetuation of historical trauma and the impacts on Native FASD rates stood as a barrier to initial partnership development. 
Elders and Natural Helpers also discussed how the government's lack of

resources toward addressing FASD represented a structural inequality that contributes to the perpetuation of historical trauma. One Natural Helper described how this structural inequality produces contemporary traumas in her own life:

There was no fucking support. Nobody even... there was nothing. There was nothing. There was nothing. You can research it and research it and research it. There are no support groups for people who love someone with FASD. None, Amanda. There are no groups where you can go to people for counseling. People will be like, "Well we can counsel you for drug addiction and alcoholism but we don't know anything about being in a relationship with an FAE person. And they make you feel like you're a fucking alien or some weirdo for being in a relationship with someone with FASD. I tried to find support out there, or someone who understood us, or who could have helped us or counseled us, and helped us finding tools and ways to make it work, to make it together, to coexist and to co-parent and to raise our child in the same household and make it work. There was fucking nobody. Nobody.

This quotes highlights how the complete lack of services for Native families suffering from FASD contribute to frustration and despair within Native communities. Shame and stigma from service providers toward Native families experiencing FASD was also a common theme from FGC members as well as members of the Portland Native community who participated in the FGC's community forums. These factors contributed to barriers faced by Native families seeking FASD services and to mistrust of government agencies.

Further, Native mistrust was also fueled by the subject matter of FASD in Native communities. One Native partner describes her initial impressions of County partners:

I sensed they were using the information against Native people instead of being of help and uplifting. History has shown that information has been used againstlike people would have their children removed if they had subsequent children and they said they had been drinking, on meth, and all of these things. I just didn't feel comfortable with putting Native people at risk. Because I'd like to think that they would be, if they are asking these questions, it's not like they should be 
interrupting family, but that the family could be honest and seek help. So I just didn't have much trust for who these people were because I never even met them.

This Native partner describes hesitance to reveal sensitive information into the hands of government. It also exposes how research around Native American substance use has perpetuated historical trauma in Native communities.

The significance of FASD in Native communities also adds further depth to Native partners' critique of the Health Department's one-size-fits-all approach to addressing Native FASD rates. This is especially true considering SBIRT's emphasis on pregnancy prevention. A Native partner stated:

It was, "You can't do that because it doesn't work." In fact, that's how we started, and it was really supposed to be a pregnancy prevention thing. And our Director said, "You know what? That isn't going to work in this community. It just flat isn't going to work. You need to do something that is trauma-informed care, that has to do with healthy pregnancies, because you are not going to stop people from having children in the Native community, that is just how that works, no matter their age. You can talk pregnancy prevention all you want, but realistically no, it's not going to happen."

Within this Native partner's critique, it is clear that the County's role in sterilization as well as the historical trauma created from Native American genocide more broadly made it absolutely inappropriate for a government agency to lead a pregnancy prevention program in the Portland Native community. Importantly, this quote also identifies the basic elements of what would become the FGC's trauma-informed model.

Throughout the formation stage of the FGC, it is clear how County partners contributed to the perpetuation of historical trauma. This created high levels of mistrust between Native organizations and the government agencies within the FGC. One County partner reflected: 
We're new people, but we represent a very visible agency that has done a lot of bad things to the community. So the mistrust or the lack of trust was for legitimate reasons and reasons that we were not aware of, because we were not aware of the history between government and Native communities. Yeah, that made it difficult to do anything about that and I think what we struggled with in the beginning. This is where my original thought was, "Let's just forget about the past. Fresh start from this moment on." But that doesn't work in the real world or with this community because I'm sure a lot of people come in and say, "Oh yeah, that's them, that's them we're different and we have different intentions." So we were basically repeating, we would have been repeating the same mistake and like make the mistrust even deeper.

This quote highlights how County partners' lack of awareness to historical trauma created by their department acted in tandem with Native American mistrust to create large barriers to collaboration between government agencies and the Portland Native community. Several FGC partners confirmed that high levels of Native mistrust of government agencies nearly halted government partnerships with Native organizations and the formation of the FGC all together.

\section{"The Shift" to a Trauma-Informed CBPR Model and the Formation of the FGC}

The Health Department's initial approach to address substance-exposed pregnancies in the Portland Native community contributed to the systemic perpetuation of historical trauma. Nevertheless, Native partners were still willing to work in collaboration with the Health Department to address substance-exposed pregnancies, provided that certain changes were made. Native partners exhibited high level of resistance, by pushing back against the oppressive behaviors of the Health Department. This required Native partners to bring their colleagues from the County up to speed on what historical trauma was and how it impacted the relationships between government agencies and Native communities. FGC members described how the educational phase of 
partnership development took place over the course of approximately eight to twelve months. During this time, Native partners shared stories during that helped contextualize the government's role in systemically perpetuating historical trauma in Native communities today. Native partners described in detail the government's role in disinvesting into Native communities, excluding Native Americans from government agencies, and privileging white-western dominated processes to their County partners within the collaborative. A County partner reflected:

I also think it helped to have so many strong organizational representatives who spoke really clearly, they are so versed at walking in both worlds and walking in the Native world and walking in the white dominated world that they are amazing ambassadors and they can code switch very effectively. I think that's another place where Native voices have really risen to the top is in them kind of understanding the system really well and saying this is what we need to make this work and then responding to that and having the direction of the project and the processes be guided by their wisdom as well.

According to this County partner, resistance was embodied by Native partners ability to "walk in both worlds" and communicate to County partners their wisdom and expertise about government's role in the systemic perpetuation of historical trauma.

Prior to this educational phase, most County partners had never been exposed to the concept of historical trauma within Native communities. FGC partners described a common theme that government's acknowledgment of historical trauma was an important first step in the FGC's CBPR approach. One Native partner stated:

And we came at it and understanding that there is a lot of distrust of the State, there's a lot of distrust of the County level. And we have to build that trust and it's really built one brick at a time. And understanding that from an agency perspective, a non-Native agency perspective is kind of hard. And we had to talk about kind of really bad/not good things to them to help them to understand. We had to talk about forced sterilization. We've had to talk about boarding school. We had to talk about...just all of those things that make a difference. You know, we had to talk about not knowing out language. We had to talk about cultural 
genocide, you know? I mean, heck we went clear back to Columbus and really kind of turned it all the way back and make them understand this is why we don't trust you and it has nothing to do with you as a person. It has everything to do with the agency that you represent. And so you put it in that framework and I think there's a lot of distrust and there's a lot of distrust for really, really, really good reason. It's not just because, "Oh, we don't like you."

This quote explains Native partners' emphasis on educating County partners about historical trauma during the FGC's partnership formation stage. By sharing stories about historical trauma, Native partners enabled County partners to acknowledge their department's role in creating and sustaining historical trauma in Native communities over the long term. As this quote demonstrates, this acknowledgement was an essential to the shift toward healing from the historical trauma process.

FGC members similarly described how partnership development helped increase County partner's knowledge of Native culture and community through teachings of the relational worldview model and the medicine wheel. Prior to their participation in the FGC, most County partners described a complete lack of experience in working with Native communities. One Natural Helper described how building understanding of Native culture among non-Native government employees was essential to building trust.

She said:

I believe that's what the barriers are between the Native community and other communities, is just a lack of understanding or respect of our culture and our ways. Understanding something is different than working in a place of not knowing. You know, when I understand who you are then for me that opens me up to trust, because there is a lot of non-trust amongst us and non-Natives. And so it's building that trust and to go back through history and show them- because we try to show our hearts at the beginning and it was viewed as weakness. So we became the weaker link and the society here in North America, just because of our compassion. It was taken as weakness, when to me it wasn't weakness at all. It was being strong.

FGC members also describe how Native partners' descriptions of relational worldview 
and the medicine wheel helped to contextualize for County partners the significance of fetal alcohol spectrum disorder (FASD) within the Portland Native community. Education about Native culture was also instrumental in helping County partners understand why the one-size fits all government approaches to addressing FASD, such as SBIRT, were not able to meet the needs of the Portland Native community.

From the starting place of government acknowledgement of historical trauma, County members learned ways in which they could adjust as necessary to prevent the continued perpetuation of the historical trauma process. One County partner said, "I'm glad that our partners at the table then forced us to redirect our efforts and again I think without that change in perspective or role, I don't think the FGC would have made it this far. If we remained stubborn about how we would do things." This quote mirrors the common theme that Native partner's resistance to government agencies through critical feedback was instrumental in shaping the process and goals of the FGC. Furthermore, this quote reflects how Native partner's resistance to the Health Department's initial approach also prompted County partners to change their role within the FGC. This required County members to follow their Native colleagues' expertise in crafting a healing process that could meet the Portland Native communities' needs. This demonstrates a willingness of government agencies to support Native protective factors instead of moving ahead with their initial SBIRT approach.

FGC members identified the significance of the FGC formation on the basis of Native resilience and resistance and County partner's willingness to change. FGC members would aim to prevent future traumas by "creating systems change" and addressing disinvestment into Native communities, Native exclusion from government, 
and white-western dominated systems. This enabled Native partners and County partners to work together in crafting a design to "work upstream" and prevent the continued perpetuation of the historical trauma by government agencies. Additionally, the FGC sought to support Native protective factors as a means of promoting healing and resilience from historical trauma in terms of Native health outcomes. Finally, the FGC sought to improve the relationship between government agencies and the Portland Native community by creating trust. All of these changes describe the formation of an innovative approach to government and Native community collaborations to address substanceexposed pregnancies and historical trauma (see Figure 5, p. 105). This approach would come to be known as FGC's trauma-informed community-based participatory researching and planning model.

\section{Conclusion}

FGC members describe how the shift to a trauma-informed model represents the beginning of a healing process in the government relationships with the Portland Native community within the FGC. A County partner recalled the feedback from her Native colleague:

She said, "You know, I think the real opportunity here is not for us to try and work on this very specific intervention, but for us to really apply and ask ourselves: what does it mean to write a new story about how government and Native communities work together and apply a trauma-informed collaborative approach to that work?" And that's how the FGC was really formed, was in that re-visioning of our initial work plan.

Importantly, this quote demonstrates that the shift in the governmental approach represented "a real opportunity" and a chance to "write a new story." The Future Generations Collaborative was formed during the initial shift when County partners 
abandoned their initial SBIRT intervention and began again with new attitudes toward collaboration with their Native partners. The FGC would develop a trauma-informed process that to support the continual shifting in relationships between government agencies and the Portland Native community within the FGC.

This chapter chronicles findings on how the Multnomah County Health Department's initial approach to addressing substance-exposed pregnancies continued the systemic perpetuation of historical trauma prior to the formation of the FGC. Furthermore, this chapter addresses how feedback from Native partners initiated a shift in the County's approach and provided critical insight into the development of the FGC's traumainformed model. Likewise, County partners' willingness to acknowledge the government's role in historical trauma, make adjustments as necessary to stop perpetuation historical trauma, and to support Native protective factors represented a major shift in the County's approach to addressing substance-exposed pregnancies. The information provided in this chapter helps to explain government relationships with the Portland Native community within the context of the FGC's formation. This chapter also sets the stage for FGC members' evaluations of the success and barriers of the traumainformed process in Chapters 7 and 8. 


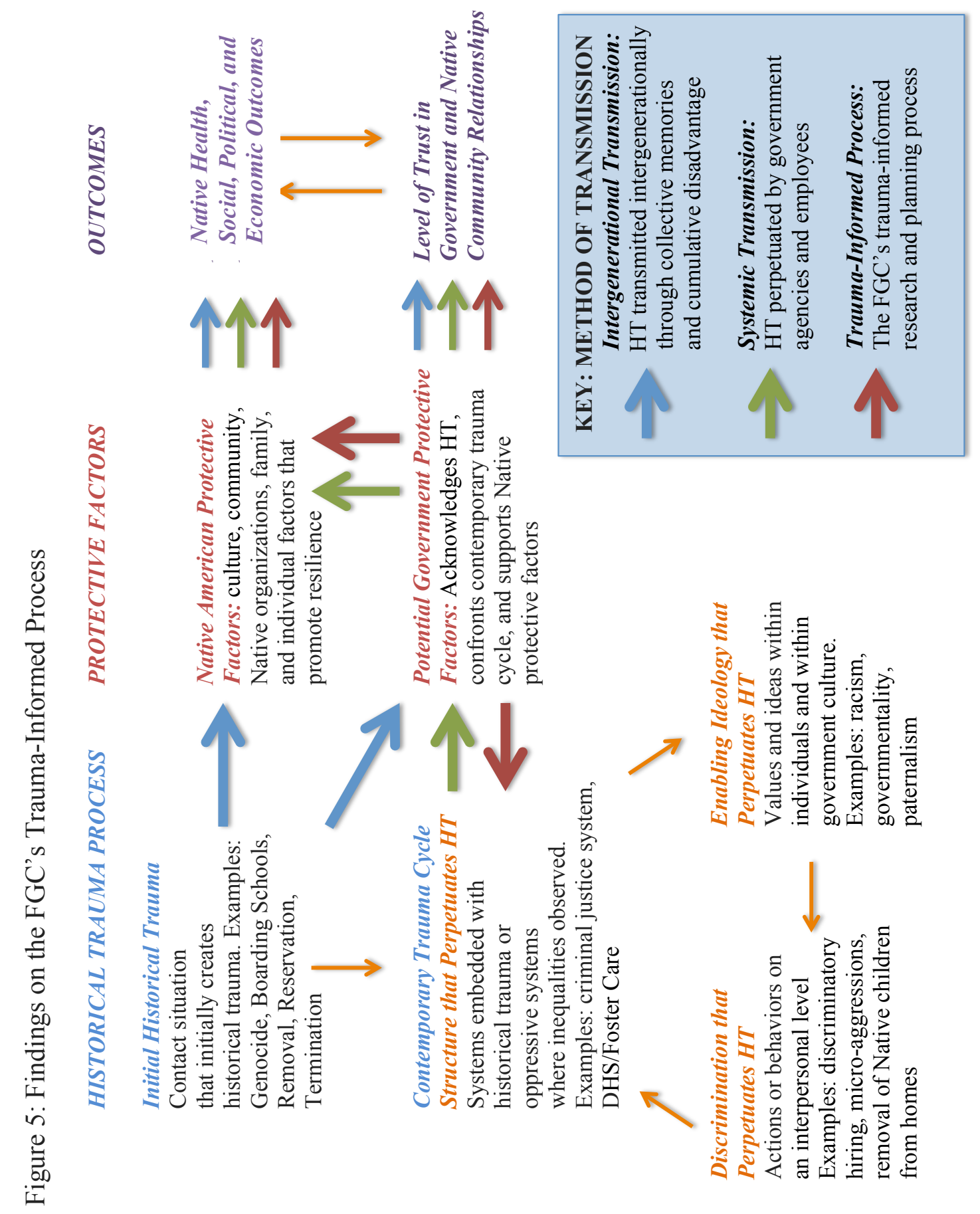




\section{Chapter 7: Findings on the Success of FGC's Trauma-Informed Model}

\section{Introduction}

As part of this study's community-based participatory research design, I asked FGC partners to help identify important areas of focus within the FGC's trauma-informed community-based participatory research and planning model. FGC partners encouraged me to examine how three features of the trauma-informed model represented a shift in the relationships between government agencies and Native communities. The three features of the trauma-informed model were described as the FGC's community capacity, collaborative partnerships, and culturally driven processes. It was clear that these features of the trauma-informed model paralleled with the body of work on community-based participatory research (CBPR) characteristics of building community capacity, equitable partnership, and cultural appropriateness respectively (Strickland 2006).

FGC members' interviews revealed connections between the FGC's traumainformed model and the structural inequalities described in the two previous chapters. FGC members described how the trauma-informed model relied on investment into building community capacity in order to address the structural inequality of government disinvestment into Native communities. The trauma-informed model's emphasis on equitable collaborative partnerships is similarly described as crucial to overcoming the structural inequality of Native exclusion from government agencies. FGC members also explained how reliance on Native-driven processes is necessary in order to counter the structural inequality of white-western domination within government agencies.

The following three sections include FGC member evaluations of how the three features of the trauma-informed model have addressed the three structural inequalities of 
the historical trauma processes. Throughout FGC members' discussions of the three mechanisms of the trauma-informed process, overlapping themes highlight the significance of partnership development and Native community engagement. The prominence of these themes throughout the following sections demonstrates the interconnected nature of the three mechanisms of the trauma-informed process. Furthermore, FGC members describe a holistic view of how the three mechanisms reinforce each other to create healing from the historical trauma process. Likewise, all of the mechanisms of the trauma-informed process should be seen as working together to impact the relationships between government agencies and the Portland Native community within the FGC. The chapter ends with discussion of the trauma-informed model's impacts on Native health as well as the partnerships between government agencies and the Portland Native community within the FGC (see Figure 5, p. 105).

Government Disinvestment in Native Communities and Building Community Capacity

FGC members described in detail how the structural inequality of government disinvestment into Native communities perpetuates historical trauma both within Native communities at large and within the FGC. The body of literature on CBPR identifies building community capacity, or building the strengths and resources of a community, as an important component of CBPR models (Strickland 2006). Within the context of the FGC, building community capacity means supporting community strengths and resources for promoting healthy Native pregnancies and healing from historical trauma. Government agencies' participation in building community capacity represents an investment into Native communities. Therefore, government agencies' participation in 
building community capacity can help to erode the structural inequality of disinvestment into Native communities and thereby slow the systemic perpetuation of historical trauma.

Under the FGC's trauma-informed model, building community capacity was discussed as an important component of the healing process from historical trauma. One Native partner said:

We're actually going through a healing process. And I think that's essential support for Native people, I think it's wonderful to be doing it in an urban setting where we have partnerships like with Multnomah County...I think we'll see that when we are our there in the horizon looking back, it's like "Remember when our work was at this point? We knew it was significant, but today we have other people, people in places of power that we've been able to create system change." Ultimately, that's what I hope we do.

This Native partner mirrors the theme that partnerships with Multnomah County help to create "support for Native people" or build community capacity, thereby creating healing from historical trauma and systems change. This section details the many ways that the FGC's trauma-informed model was able to successfully build community capacity. Building community capacity was described holistically by FGC members and included building community capacity within the FGC itself, within government, and within the Portland Native community.

Beginning with the FGC's formation, FGC members frequently described partnership development as an important element of the FGC's trauma-informed approach to building community capacity. This included an initial educational and trustbuilding phase of partnership development, described in the Chapter 6. Thus, the educational phase of partnership building laid the groundwork for County partner's highlevel understanding of how the historical trauma process functions. FGC members similarly described how partnership development helped increase County partner's 
knowledge of Native culture and community through teachings of the relational

worldview model and the medicine wheel. This increased the capacity of County partners to act as authentic allies and advocate for the Portland Native community within various governmental organizations. FGC members described how this level of advocacy by County partners contributed to an increased awareness about historical trauma and the needs of the Portland Native community with governmental agencies outside the FGC.

On a personal level, FGC partners also described significant capacity building in their own lives. FGC partners frequently described participation within the FGC as educational, providing professional and leadership development opportunities, as well as opportunities for healing. One County partner explained, “I'm going to say that I'm extremely proud of the work we have done and my contribution to the work and that this is really career defining experience for me and has been personally and professionally transformative." Exemplified here is the common theme that participation within the FGC provided a transformational experience for FGC partners. County partners often emphasized how participation within the FGC increased their personal and professional capacity by learning how to "trust the process," prioritize relationships, and engage with Native communities and culture. Native partners often emphasized how work with the FGC has been "affirming" and "inspiring" to their already well established personal and professional commitments to the Portland Native community. Native partners also tended to emphasize how participation within the FGC was healing within their own lives, based on their personal experiences of historical trauma.

Several FGC members described how the FGC's trauma-informed partnership development process built community capacity by providing an opportunity for Native 
organizations to work together collaboratively. FGC members explained how certain

Native organizations have been forced to compete with one another within a climate of limited resources. One Native partner explained how the FGC helped to "build bridges" between Native organizations by modeling trauma-informed collaboration. She stated:

There were a lot of things politically — even amongst Native agencies in Portland - that kind of were split. This year was the first year that we worked on an event together that was a collaboration between all the providers. It's the very first year, ever. And I think the reason for that was the FGC has been modeling behavior, collaborative behavior, that kind of has provided the agencies with some insight to, "Oh, it's cool if we all work together. This is what can happen if we all work together." And we all serve the same people. When we all work together everybody gets help, everybody gets served, and everybody gets something...And I think FGC had a big part in that because we have been a collaborative now for a really, really, really long time and we've been building that relationship and having collaborative efforts and didn't hurt that our two CEO's were, you know collaborating on other things as well in the community. So modeling that collaborative behavior has really helped agencies who have had histories of 'us' and 'them' kind of thing go on, to come together and go, "No this what you guys do great." And we look at them and go, "No, this what you guys do great!" You know? And we understand that we each do great and we compliment each other. And it's complimentary, we're not competing.

This Native partner demonstrates that partnerships building within the FGC had a positive impact on partnerships between Native organizations outside of the FGC. Based on the successful modeling of collaboration within the FGC, FGC members described how partnership development within the FGC served to help maximize resources and strengths in order to better serve the Native community both within and outside the FGC.

FGC partners often discussed how the successes of the FGC's trauma-informed partnership development acted as a basis for the high level of engagement of the Portland Native community within the FGC. Native partners explained that the FGC has been more successful in engaging the Portland Native community than nearly all collaborative partnerships before. FGC partners described how the "hard work" of trauma-informed 
relationship building was an instrumental foundation of the FGC's effective and efficient outreach strategy to the Native community. Similarly, Native partners describe how the deep personal investments made by FGC partners from Native organizations and County organizations alike contributed to the recruitment of Elders and Natural Helpers, or community members with active roles within the FGC. A Native partner stated:

I think another reason why the FGC is successful is because, certainly all the community partners, their work pays them to be involved. But I think everyone goes over and beyond to make sure that the work gets done, because of their workload, their full workloads already. I think that contributes to the success right? It's the heart part, so I think that contributes. I think that our people gravitate towards positivity, our people gravitate towards culture and I think that's why the Elders and Natural Helpers gravitate towards this project and even when their not getting paid... That's why they gravitate towards this projects: it's positive, it's productive, it's proactive, it's based in culture, they get to give back, they feel needed, they feel important.

Several FGC members described the common theme of the high levels of investment made by County partners and Native partners in terms of time, energy and "opening hearts". This high level of investment helped to create a positive and productive culture within the FGC that then attracted participation from Elders and Natural Helpers.

As part of the FGC's trauma-informed model, Elders and Natural Helpers are a group of Portland Native community members who were recruited and trained for roles within the FGC. This effort was grounded in the FGC's goals to create greater community representation and build community capacity within the FGC. Most Elders and Natural Helpers were recruited during Portland Native community events hosted by the Native American Youth and Family Center (NAYA) and the Native American Rehabilitation Association (NARA). Other Elders and Natural Helpers came along with 
family or friends to the initial FGC training. Once Elders and Natural Helpers became engaged within the FGC, they also became instrumental in building community capacity.

Many FGC members focused on the ways in which Elders and Natural Helpers are "at the heart" of the building community capacity. Likewise, FGC members attributed much of the FGC's success in community engagement to the work of the Elders and Natural Helpers. As part of the FGC's trauma-informed model, Elders and Natural Helpers were brought in and trained in historical trauma, FASD, and the various roles of the FGC's community forums. The community forums were designed to collect information on Portland Native community members' knowledge of FASD and historical trauma. In combination with the Gathering of Native Americans (GONA) - a Native healing curriculum designed to address substance abuse in Native communities - the community forums were also designed to inform future FGC initiatives for promoting healthy pregnancies and healing from historical trauma within the Portland Native community. During the implementation of the community forums, Elders and Natural Helpers took on the vast majority of leadership roles.

Elders and Natural Helpers also helped build the FGC's community capacity by increasing recruitment and reducing the barriers that often exist between researchers and subjects within Native communities. The FGC's eight community forums were well attended with over a total of over 150 participants. One Natural Helper described:

And the Native community is now starting to see that we are trying to address these issues. We're trying to create programs around them. And I know a lot of them right now are really skeptical because we've been presented with a lot of programs over the years and some of them did work and a lot of them didn't really work. And that's why we are trying to create new programs. But I think they see the difference because we did go to the Native community and ask for information and it wasn't outside non-Native people asking them these things. 
They went into the community and found Natural Helpers and Elders within the community, trained them about how all these things that we're focusing on, and sent them back out into the community to ask them about the information that now we're using to build the programs around, the GONA and the other information that will come after that. And I think they really take that into account because it's different than what programs we've been presented with before. But I think there is still a skepticism kind of waiting to see how effective it's going to be.

This quote reflects common themes that many barriers have stood in the way of Native participation in research and planning settings. FGC members describe how Native communities often become disengaged from research and planning processes based on the fact that researchers and planners are often outsiders from the Native community. Additionally, barriers to inclusion are also created by the fact that FASD is often stigmatized and that many initiatives have failed to follow through with support for Native communities. This quote demonstrates that Elders and Natural Helpers roles in going to the Portland Native community and asking for their views on historical trauma and FASD built community capacity by successfully engaging the Native community in the research and planning process.

After the community forums, Elders and Natural Helpers again contributed to building community capacity by participating in the FGC's two Meaning Making Meetings, where the results of the community forums were brought back to the Portland Native community for validation. Some Elders and Natural Helpers also began attending regular FGC planning meetings, thus helping to shape the direction of the FGC. One County partner explained the significance of Elders and Natural Helpers' contribution within planning meetings, stating:

I believe that this project has gone beyond what many projects like this do with the model of the Natural Helpers and Elders. I see this really growing. I don't see 
this just staying where it's at. That we really have built capacity for this community planning, because it's not just, a lot of these community plannings take place with CEO's, with other managers of these programs so that the people that the ultimate decisions are made are based on are not the people in the community. It's based on what people think the people in the community need. But this project has turned that upside down with the Natural Helpers and Elders, who have a very strong voice in where this project is going and the decision making and how we're going to go forward from here. So the agency voices are there just as strong as the community itself. And so I think that it's a huge impact to developing community — actual community capacity.

Elders and Natural Helpers were able to contribute a "strong voice" and influence decision-making within the FGC, thereby making the FGC much more effective in its work within the Portland Native community. This quote also illustrates the common theme that the FGC is building community capacity through development of a traumainformed planning model that is grounded within the Native community.

Elders and Natural Helpers also participated in sharing the results of the community forums during partnership meetings and at conferences. One Natural Helper described her experiences attending a health board meeting, where she advocated for the needs of the Native community to a large audience medical professionals and social service employees. She stated:

It was letting the doctors know our point of view of where our difficulties lie with communication. So that was another part that I thought was really good because health is a big deal when you're pregnant. And when you get the discrimination feeling and you get hard feelings against a doctor and you don't want nothing to do with another doctor. Or it's just the language itself. You know, there's a lot of barriers that prevent a lot of Native Americans from getting that proper health care. And I feel it's a big deal, it's a very big deal when they hardly understand or they get shot down because they use slang or they don't understand fully and completely. So it gets blown over like, "Oh this is what I'm going to give you, take two and call me in the morning."...Afterwards they were all coming up and was asking questions. I was nervous. I barely remember what I was saying but after they left it all came back to me. And then I was like, "Wait, I said that didn't I?" But knowing that there was doctors, nurses, WIC people [The Special Supplemental Nutrition Program for Women, Infants, and Children], social 
workers, you know and surgeons in there. I was like, "Whoa." And then people online, like "Oh my goodness". People that were in school to become doctors, nurses, social workers, everybody that was there. There were people in schools like Harvard and Yale. There was big schools.

This Natural Helper's story reveals the significant impact Elders and Natural Helpers had in building community capacity by educating medical professional and social service agencies on best practices of working with the Portland Native community. The impact of Elders and Natural Helpers roles within this type of outreach to non-Native organizations was so profound that the FGC received a Public Health Heroes Award in April 2014.

Another way in which the FGC worked to build community capacity was through hosting a Gathering of Native Americans (GONA). The GONA curriculum was first developed by the Substance Abuse and Mental Health Services Administration (SAMSHA) as a culturally relevant, evidence-based, holistic approach to addressing substance abuse in Native communities. As part of the FGC's trauma-informed approach to building community capacity, the GONA represented a three-day healing event that incorporated education about historical trauma and FASD and was grounded in Native culture. The goals of the GONA included education, skill building, and collaboration in order to facilitate personal and community healing from historical trauma and FASD. In this way, the GONA event in itself marked a significant investment into building community capacity within the Portland Native community by providing "opportunities for healing" and fostering what FGC members described as the Native traditional values of belonging, mastery, interdependence, and generosity. While the vast majority of GONA participants were Portland Native community members, representatives from 
various government agencies and Native organizations were also in attendance. The number of GONA attendees who signed in totaled over 188 people each of the three days.

Elders and Natural Helpers also took on important facilitating roles during the GONA. After the GONA, FGC members gathered together to debrief the event and evaluate how it went. FGC members reported that a number of community members were inspired by the role of Elders and Natural Helpers at the event, stating that seeing the faces of Elders and Natural Helpers and hearing some of their stories gave them strength and motivation to be more involved in the community and within their own lives. One Native GONA participant described the impact Elders and Natural Helpers' participation during the GONA, stating: "I pretty much gave up on getting my kids back. But after hearing the stories here, I am motivated to work hard on myself and try to get my kids back. Thank you for this opportunity." This quote reflects the common theme among FGC members that Elders and Natural Helpers helped build community capacity by acting as positive role models within the Native community.

Elders and Natural Helpers learned skills, gained work experience, and professional development opportunities within the FGC. This type of learning and training contributed to Elders and Natural Helpers ability to "walk in both worlds" and act as leaders and advocates for the Portland Native community. FGC members often cited the fact that Elders and Natural Helpers began self-organizing and implementing their own weekly meetings as an example of this increased capacity. When asked to what extent the FGC had built community capacity, one Native partner stated:

I see it all the time, just a little bit ago I told you we host a meeting every single Wednesday night with the Elders and Natural Helpers, who are a learning cohort that came out of the Future Generations Collaborative that are self-organizing and 
expanding their skill and their ability and identifying ways that they can continue to work in the community. And I'm calling it healing work. I think if you ask different people they might call it different things, but I think you'll hear that thread of healing going on, and capacity building. I refer to it as the power of one and the power of five. The power of one is you have to be the change that you want to see. We could sit around and gripe about stuff all day long, but until we are really committed to a change and leaning in ourselves, then we can't-we don't really have a lot of room to gripe. But once we've leaned into that work and we're gaining knowledge around how to move it, we start to tell people. And if we tell five people those five people are going to tell five more people and that's coalition building, and that's kind of what's happening with the Elders and Natural Helpers.

According to this Native partner, building on the skills and resources of Elders and Natural Helpers has contributed to "coalition building" between members of the Portland Native community. This Native partner's statement also clearly identifies the common theme that working together, expanding skill, and learning within the FGC has helped to clarify many Elders and Natural Helpers goals and fostered a stronger commitment to "work in the community." Importantly, professional development for Elders and Natural Helpers is described as both building community capacity and as "healing work."

Frequently, members of the FGC described the healing opportunities for Elders and Natural Helpers as one of the most significant forms of building community capacity that the FGC contributed to. While many FGC members describe the entire traumainformed process as healing, they also identify specific and intentional "opportunities for healing" within the FGC. For example, FGC members explained that throughout their participation in the FGC, Elders and Natural Helpers were transformed through education about historical trauma and FASD, increased self-awareness, self-esteem, empowerment, and commitment to family and community. One Natural Helper explained the ways in which she has been personally impacted by her work within the FGC: 
I've been through some struggles since I started with the FGC, like I said it has helped me in my recovery and at in times kept me sober, but it has also opened my eyes to sometimes what's more important_and that is some of the relationships that I've made — not what's more important, maybe it's given me something that I've missed because I've always had an issue creating those strong bonds with females. And we have a lot of strong women in the FGC, and been able to create this sisterhood together which has helped me. As women we need those strong relationships...I've gained so much, just beyond the educational purposes. It's mostly all the personal stuff that goes on, like I said the relationships. Like comparing my life and the work that we do here-kind of like where I'm going wrong and what I need to change in my personal relationshipseven though I haven't changed a whole lot. I know, it's given me some clarity, so...yeah but mostly just the personal stuff has been really awesome to see and grow. A lot of growth.

Several FGC members cited the theme that participation within the FGC supported the recovery of some FGC members who had first-hand experience with substance abuse. Additionally, participation within the FGC provided a sense of community for Elders and Natural Helpers and especially strong relationship with other women. This quote also explains how healthy relationships within the FGC also promoted healthy relationships outside the FGC. Thus participation FGC's "healing opportunities" supported the resilience of Elders and Natural Helpers.

FGC members also described how often Native community members would express interest in becoming an Elder and Natural Helper with the FGC in a future cohort. FGC members again cited this as an example of the high level of community engagement achieved by the FGC as well as the positive role modeling of the Elders and Natural Helpers at FGC events. One Native partner expressed the ways in which Elders and Natural Helpers have succeeded in building community capacity, stating:

They're serving as positive role models. Other people are seeing them in a different way on several levels. In a different way personally, where they're wanting something better for themselves... And I've actually had calls from other community people saying how can they be an Elder and Natural Helper, right? So 
that's awesome and it's exactly what we wanted them to do is to cultivate this level of excitement within the community...I think that the community forums and having the Elders and Natural Helpers facilitate those, was phenomenal because of how it empowered them, right? It totally empowered them and it gave them an opportunity to feel important, to feel needed and wanted, and their skills were validated, and so it really did a lot for them personally, right? And then the community got to see them in those roles where even if some of their friends came to a community forum and they saw them in a different role, it's like wow! So it made them feel the self-esteem, the self-worth, the self-confidence. I mean everything was just like boosted. And again those are all building blocks so when we talk about community development and community capacity building and all that kind of stuff.

This Native partner reveals the common theme that opportunities for building Elders and Natural Helper's capacity on the personal level strengthened Native community capacity more broadly by modeling healing and leadership.

Similarly, FGC members frequently described how participation within the FGC has helped Elders and Natural Helpers act as positive role models within their families. As part of the FGC's trauma-informed model, children were often included at FGC meetings and events. FGC members frequently described this characteristic of the FGC as building community capacity as children absorbed the content of the FGC and watched their parents and engage in healthy behaviors. One Natural Helper described:

I would like to see my family learn about these things, because I'm the type of the guy that if I can't take care of myself, how am I going to be able to take care of my family? And so if I can't take care of my family, how am I going to be able to take care of the community? Because it all has to start from within. You've gotta reach yourself, then your family, then the community because you have to be able to have that support of your family to be able to stand up in the community and recognize that. You need to be able to have your family stand behind you, really. Far as I'm concerned, if my daughter can answer a question that I happen to miss from somebody, that's a good start. If my wife can answer a question better than I can because I'm off answering a separate question, that's another good start. Because I don't want myself to be the only resource that people need out there, I want to be able to have my kids involved so that they can see, so that they can answer the questions, help me answer questions really. You know, I need that backing. 
This quote demonstrates how participation within the FGC often promoted healing among FGC members who are parents. Furthermore, this quote exemplifies how the FGC provided opportunities for personal empowerment that in turn supported the empowerment and engagement of Native families. FGC members frequently described the high number healthy pregnancies of FGC members - a total of six during my participant observation — with a 100 percent breast-feeding rate as another form of building community capacity that the FGC contributed to. In this way, participation within the FGC was seen beginning to change community norms and promoting healthy pregnancies within the Portland Native community. Therefore, the FGC could be framed as an intervention itself by the ways in which community engagement and healing are held central throughout the trauma-informed research and planning process.

\section{Native Exclusion from Government Agencies and Native Representation}

Another structural inequality that FGC members discussed at length was Native exclusion from government agencies. Here again, FGC members connected Native exclusion to the systemic perpetuation of historical trauma within the FGC as well as within the broader Native community. This theme from FGC interviews parallels the CBPR literature on equitable partnership (Strickland 2006). According to CBPR scholars, equitable partnership helps to ensure Native representation within research settings (Strickland 2006). Similarly, government participation in equitable partnerships with Native communities in the research and planning setting weakens the structural inequality of Native exclusion from government agencies. Thus equitable partnership and Native 
representation in collaboration with government agencies can aid in dismantling the systemic transmission of historical trauma.

FGC members commonly discussed equitable partnership as a critical component to their trauma-informed model and as essential to healing from historical trauma. For most FGC members, equitable partnership within the trauma-informed setting actually meant that the Portland Native community drove the research and the planning process. In fact, many FGC members critiqued the notion of "equal partnership" within the relationship between government agencies and Native communities. One Native partner explained what equal partnership means to him:

For that relationship to be equal it'd be like pretty much let the Native people decide what to do because equality between the State and Federal governments and Native Americans looks different. I mean, it would look different, to me to have equality would be to put the power in the hands in the Native Americans and not let the State really have any say in what they're doing. It'd be to just give them the money and let them do what they see fit for their own community, that would be an equal relationship... [Because] based on this history, and the number of resources available for Natives, that are given by the State and the Federal government in proportion to other communities and other ways that the State and Federal governments fund. It's really disproportionate and the Native community has been deprived so much that if there is a program for Native Americans they need to be given everything. They need to be given all of the power and all the resources to do, and that still doesn't even like scratch the surface of the debt that they owe to the Native community.

This quote demonstrates the common theme that taking a trauma-informed approach to equitable partnership means acknowledging the unequal relationships that have existed throughout the relationship between U.S. governmental agencies and Native communities. FGC members describe how this holistic view of equitable partnership should influence roles of government agencies and Native communities within the collaborative setting. One County partner similarly detailed this approach as striving toward a "net equality" 
that takes into account legacy of historical trauma and structural inequalities imposed upon Native communities by government agencies.

FGC members also described the importance of grounding the community-based participatory research and planning process in high levels of Native representation. This provides an opportunity for achieving "authentic partnerships" that honors the selfdetermination of Native communities. One Native partner explained:

If there's Native with State and County systems being funded in an administrative capacity but it is for Native people then it really needs to be in the language and the voice of that group of people and that dynamic and the meetings style needs to look at that.

Achieving balance within the partnership between government agencies and Native communities where the goal is to serve Native people means that Native voices are prioritized and that research and planning is grounded within the Native community and culture.

Many FGC members described the FGC as successful in achieving equitable or authentic partnerships based on the high degree of Native representation within the FGC. FGC members commonly described the FGC's trauma-informed process as "for community, by community" or as a "Native-owned" and "Native-driven." This point was illustrated by the numbers of Native people engaged within the various roles of the FGC. One Native partner explained:

I value the fact that our monthly organizing meetings are open to the Elders and Natural Helpers who are every day community members who come in, they're voice matters, their opinions influence our direction many times. I think that's a wonderful example of providing that voice. The fact that we have three Native participants at a national level speaks volume to that ability, we have more than eight Native-serving organizations at the table and very few mainstream or majority type organizations that are serving, and they're respectful when they are at the table. They don't try to dominate the conversation or bring in their priorities. 
They're there to learn and listen from our community.

The high degree of Native representation achieved within the FGC is demonstrated in this Native partner's evaluation. She describes Portland Native community members, including Native partners from eight Native serving organizations as well as Elders and Natural Helpers as taking part in the FGC's monthly planning meetings. Furthermore, this quote explains the significance of three Native partners representing the FGC at the national level. Many FGC members also emphasized the high degree of Native representation within the FGC's trauma-informed research and planning process by discussing how the Portland Native community has been asked to participate in FGC events such the community forums, the Meaning Making Meetings, and the GONA.

FGC members not only discussed the importance of Native representation in terms of numbers of Native community members involved in the FGC but also emphasized Native representation with regard to the level to which the FGC integrated Native voices into the trauma-informed process. FGC members described how Native voices were heard within various roles of the FGC. Elders, Natural Helpers and Native partners also share their knowledge, experience, and expertise throughout the traumainformed process. One County partner discussed:

I think it was a big improvement than before the FGC's time. I think more and more the Native concerns and the Native voice were gaining visibility in the Health Department. But I think there's a lot of room for improvement just because it will just take a long time to break down the barriers that have prohibited this type of collaboration working in the past. I was really impressed that within the agency there were folks representing four or five different Native organizations and multiple tribes. And I haven't been in Portland that long but that's unprecedented to me to have such internal diversity especially in getting representatives from NAYA and NARA together. I think that's like a huge milestone. 
Exhibited here is the common theme that the FGC has achieved "unprecedented" representation of Native organizations within government agencies in the Multnomah County area. Many FGC members point to the inclusion of Elder and Natural Helper voices within the FGC's planning meetings as another sign of how the $\mathrm{FGC}$ has successfully included some of the "internal diversity" within the Native community.

Additionally, Native community members' voices were shared at the community forums, the Meaning Making Meetings and the GONA. When asked if the FGC represented Native voices and interests, one Natural Helper explained: "Yeah, because it is based on the Native community. Yeah, it's indigenous, I feel like our voice is what needs to be heard because following up with all the forums that we put on, it's a huge thing in the Native community." This quote mirrors the common theme that the community forums and other FGC events have provided an opportunity for the inclusion of Native voices from the broader Portland Native community. In all of these examples, FGC members frequently describe how the FGC's trauma-informed process is grounded in the voices of the Portland Native community.

Moreover, FGC members frequently describe how Native voices also influenced the FGC's decision making. Initially, FGC members describe how the trauma-planning process took shape based on the Native expertise on what approaches worked well or were inappropriate for the Portland Native community. As the term, "process" infers, the FGC's trauma-informed model was designed to evolve over time as informed by Native community needs. Therefore, FGC members describe how Native voices and expertise was continually integrated into the decision-making around the FGC's goals, values, and methods as the trauma-informed process unfolded. One Natural Helper said: 
One of the things that they did after I talked about the lack of involvement of the Natural Helpers is that they did start assigning people to do positions and they did start letting them know that these are the positions that we're going to have at the GONA conference: facilitators, co-facilitators, greeters, support people. Just all these positions we're going to be filling during the GONA conference and they laid them out and stuff and were asking people for volunteers. And also asking people to do presentations as facilitators. During the Spirit of Giving Conference they asked the volunteers to put together a presentation to present to NARA for the Spirit of Giving Conference and asking them to do that. So it was, based on what I said, they started to get the Natural Helpers and Elders more involved. The retreat was put together after I mentioned this as a way to get everyone up to date and everyone to come together and spend some time together as we are giving assignments and doing updates.

Importantly, a Natural Helper's feedbacks lead to the increased engagement of Elders and Natural Helpers within the trauma-informed process. The quote describes a common theme that the successful integration of Native feedback into FGC decision-making continually supports the equitable partnerships within the trauma-informed process.

While FGC members emphasized the importance of Native representation within the context of collaborative partnerships between government agencies and Native communities, they did not necessarily diminish importance of government participation.

Most FGC members highlighted the importance of strong partnerships between government agencies and Native communities and felt that the government had a responsibility participate in collaborative research and planning. One County partner explained:

So in the case of the FGC, I think what I want to see is, and I think what should exist is actually more power should exist in the voices within the Native community and that government should respond to that by sharing more power more decision-making and creating - and I think if anything were equal-it might be a collective sense of responsibility for addressing health inequities, like everybody has a role in that, and everybody is a partner in that, but where the power should lie is really with the Native community and what our job as government should be and what I'd like to see us get better at doing is actually creating more equitable circumstances so that more and more power is in the 
community to address their own health issues. Whether that's funding or creating educational systems that work better for Native communities.

Many FGC members described how government agencies needed to be responsible for addressing health inequities experienced by Native Americans in partnership with Native communities. Some Native FGC members expressed a hesitance toward government inclusion into initiatives designed for Native communities generally. However, when it came to discussing government participation within collaborative setting within the FGC, most FGC members described how authentic partnerships with government helped improve the capacity of research and planning.

FGC members emphasized that the power, influence, and resources that government agencies hold make collaborative partnerships necessary to meet the needs of Native communities. Unfortunately, the historical trauma and contemporary traumas caused by the structural inequality of Native exclusion often meant government's role in Native communities was more oppressive and destructive than useful. According to FGC members, one factor that made the difference in the FGC's trauma-informed model was the fact that County partners were "like-minded and like-hearted" and had "the people are at the heart of the project." One Natural Helper said:

But there's got to be non-Native people because we live in a world where we're all together. We're all together here. It's never going to be an all Native, there's always going to be non-Native people involved. But they have our people's best interest at hand and they have personal connections to our community and our people, like you and your husband.

As noted by many FGC members, this quote establishes the value of non-Native allies who have Native interests at heart and who have connections to the Native community. 
Many FGC members stated that County partners within the FGC did indeed have the Native community's best interests in mind.

FGC members also described several other factors that contributed to authentic County partnerships. This included County partners the ability to listen to their Native colleagues. It also included County partners' willingness to do things differently and to share leadership and power. In other words, the FGC's trauma-informed process called County partners to participate in non-hierarchical relationships. One Native partner explained:

I think that at no point during FGC have I ever seen our County and State partners not want to walk side by side. Or not want to be a part of it and when I say that, they truly are invested in what we're doing and really have a good strong belief system of "It really is going to make a difference."... We could all sit at a round table and it could be United Nations because of the way that we have built up the interpersonal relationships amongst each other, not just the working relationships.

This quote illustrates a common theme among FGC members, who described County partners as working "side by side" with the Portland Native community within the FGC. Importantly, this point does not necessarily contradict FGC members' description of the need for strong Native representation within the FGC. Rather, FGC members tend to describe the need for strong Native representation and non-hierarchical relationships as complementary characteristics of equitable partnership.

County partners and the Portland Native community were commonly described as sharing roles within a Native-driven process. FGC members commonly described the County's role within the FGC as administrative in nature. Therefore, County partners are often responsible for roles involving transportation, organization, communication, meeting facilitation, and funding. One Native partner describes how and why the County 
members were nominated for their administrative role within the FGC of handling matters of funding:

Everybody had a say in, "yes". In fact, it was asked, "Do we need to give it to this one or that one or the other one." And you know, we all said, "No. The money came from you. It's best if you stay with it, you guys have control over the money at that point. You have that capacity. You are an entity that can do that." And we all said, "We want you to do this as a group." And we actually voted about that. So that in and of itself. They've never had that, "I'm going to be big brother" kind of scenario, unless they've been asked to. And there have been a couple of occasions where we have said, "You guys need to be big brother." Because of the politics of getting certain things and making it work...so we use that quite a bit to our advantage and were allowed to do that and I think that's important. But nobody is ever the biggest dog in the room. It just, whoever needs to be the biggest dog, "Go be the biggest dog." Just like, send the oldest grandkid to ask Grandma if we can have popsicles, "Go ask Grandma if we can have popsicles." It's the same. It's kind of that same scenario of: how do we get the most bang for our buck? You know, and who can be the lead schmooze and who has the best relationship with these people.

While County partners sometimes take the role of "biggest dog," it is only when it is agreed upon within the collaborative to serve the best interests of the FGC. Partners from Native organizations likewise take the lead when they have the most capacity to do so. In this way, leadership is shared within the FGC and roles are agreed upon as a collaborative.

\section{White-Western Dominated Processes and Native-Driven Processes}

The third structural inequality that FGC members focused on was government agencies' reliance on white-western dominated processes. According to FGC members, white-western dominated systems are significant because they continue the perpetuation of historical trauma within Native communities generally and within the FGC. The CBPR literature makes similar connections, calling out white-western dominated processes within the research setting as having profoundly impacts on Native communities 
(Strickland 2006). The body of literature on CBPR often calls for "culturally appropriate" research processes that embody Native culture and help reduce the negative impact of white-western dominated processes (Strickland 2006). Similarly, government participation in culturally appropriate processes or Native-driven processes within collaborations with Native communities can aid in breaking the structural inequality of white-western dominated processes. In this way, culturally appropriate and Native-driven processes within collaborative settings between government agencies and Native communities can help weaken the systemic transmission of historical trauma.

FGC members emphasized the essential nature of culturally appropriate and Native-driven processes to their trauma-informed model. As part of the FGC's traumainformed model, FGC members described cultural appropriateness as processes that are grounded in Native culture and community. At the same time, FGC members commonly took issue with the term "cultural appropriateness" within the relationship between government agencies and Native communities, stating that this term was counter productive and an appropriative term for Native traditional processes. One Native partner stated:

It's a new way of doing old way business. As tribal people, we know all this stuff common sense, intuitively. It's how we come together as communities and that was disrupted for a period of time and in a way we are being given and acknowledged for this opportunity to come home to our old ways. That's how I view it, and it's exciting and it is a healing component for our community too.

FGC members widely shared this theme that the FGC's process has been successful not by innovating new methods of cultural appropriateness between government agencies and Native communities. Rather, FGC members describe the FGC's success in cultural 
appropriateness to the high level of integration of Native culture and traditional ways into the trauma-informed process.

Native partners representation within the FGC was often described by FGC members as central to forming a trauma-informed process that was grounded in Native culture. FGC members explained how Native partners provided expertise and technical assistance in order to guide the trauma-informed process in a number of ways. Common examples were Native partner's role in determining the scope and goals of the FGC, choosing best practices and processes for planning meetings, training County partners on historical trauma and the Portland Native community, recruitment and training of Elders and Natural Helpers, and facilitating the engagement of the Portland Native community. One Native partner explained the FGC's "Native-owned" process since the collaborative received initial funding from City Match, the National Organization of Urban Maternal Child Health Leaders:

I know this from a community perspective because I've heard it with my own ears that this is the only project that I believe we've engaged in that truly is of the community. I mean since its inception in City Match, it's morphed into this project that is the community. So, I mean it's a Native-owned, Native-inclusive/ guided/driven project. I mean, I think it's a good project because we have those voices that kind of lay out the scope of where we have to navigate to be successful. Provide us with some expertise or technical assistance, like "Nah, that's not going to fly" or "These are the hoops we have to jump through," or "Perhaps we need to meet with these people or those people, perhaps our presentation should highlight these important things." It's all a matter of what we discussed earlier of kind of finding a blend and being successful.

Native partners were essential in crafting the FGC as a Native-driven and a Native-owned project and facilitating trauma-informed process where Native culture and Native community is "at the center". 
FGC members described several contributing factors that supported the FGC's Native-driven processes. First, Native cultural values and ways of knowing were prioritized throughout the FGC's process. FGC members often cited examples of Native values present in the FGC's trauma-informed process, including: historical trauma, the relational worldview, and emphasis on healing and the medicine wheel. Several FGC members explained how even the name, "Future Generations Collaborative" represents the deep integration of Native traditional teachings into the FGC. One Native partner stated:

For example a traditional teaching and a traditional approach is always looking to the future and always planning for the future generations. And even our name is embracing that teaching. Even if people say, "FGC," but if you really look at it: Future... Generations... Collaborative. The name in itself is a traditional teaching, and what we're trying to do again is that we're trying to change norms within the community where the norm isn't to drink during pregnancy, the norm isn't to have these unhealthy behaviors, you know all that kind of stuff. So the name itself is a cultural approach.

This quote indicates that Native traditional teachings inform the FGC's cultural approach to addressing substance-exposed pregnancies. This helps to explain the FGC's traumainformed approach to addressing substance-exposed pregnancies, which focuses on strengths-based approaches. FGC members frequently explain how emphasis on healthy pregnancies, resilience, breaking the cycles of FASD, and using Native culture and community as prevention all represent strengths-based approaches that are reflexive of Native teachings. The integration of Native traditional teachings also explains the FGC's emphasis on positivity, respect, and inclusiveness within the trauma-informed model.

FGC members do not only express the importance of integrating Native traditional knowledge and worldviews into the cultural approach of the FGC, they also 
discuss integrating Native cultural practices into the FGC's trauma-informed model. FGC members cited many different examples of ways in which Native values and practices shaped the methods used during FGC meetings and events. For example FGC members described the significance of the FGC using less rigid meetings formats, focusing on relationship building, emphasizing process over outcomes, and "slowing down" to honor and include Native voices. One Natural Helper explained:

That's culturally appropriate right there, what we are doing is very important for the community. Like I told you, warriors used to sit in circles and they would try to find a way to figure out peace and they would take hours doing it, sometimes days, weeks. They had no set time limits. They didn't have anywhere important to go, and the people from their villages understood those guys are taking care of something very important right now. Leave them be. They're discussing whether or not they want to kill each other or have peace.

This Natural Helper's quote illustrates the common themes of how Native traditional values and practices are integrated into the FGC's trauma-informed process — such as of honoring Native voices and showing respect to pertinent issues within the Native community.

FGC members also frequently expressed the importance of using Native ceremony and practices throughout the trauma-informed process. Many FGC members emphasized how these traditional practices have been proven as the most effective way of engaging Native communities in the healing work of trauma-informed research and planning. A Natural Helper explained:

I think that if we are not culturally appropriate in our dealings that some people may not be able to identify with what's going on or feel comfortable - like Natives. We joke and we say, "Oh we make a ceremony out of everything." But we really do. It's like, "Oh God, we always have to bring out the medicine and smudge everything down. We always do that." But we do, but we do. And acknowledging that and being willing to say, let's smudge before we have the meeting. Let's offer smudge for those who want to go outside and do it, is huge. 
And I think that a lot of people will open up and feel more comfortable if we keep doing those kinds of things.

This quote demonstrates how integration of Native traditional practices and ceremony helped increase Native engagement by making the Native community "feel more comfortable". Common examples of Native ceremony and practices that are effectively integrated into FGC meetings and events include: sitting in circles, opening meetings with blessing, introductions and check-ins, as well as integrating smudging, sharing meals, singing, and storytelling.

Additionally, FGC members often cited efforts to make the FGC more accessible as another way Native values and practices have shaped FGC processes. Frequent examples included providing food, transportation, childcare, and participation incentives at FGC meetings and events. Providing stipends for Elders and Natural Helpers as well as childcare, meals, and transportation were often described as a critical to facilitating accessibility within the FGC. Culturally specific and non-stigmatizing language choices were also used within FGC processes to facilitate Native inclusion, especially considering the subject matter or "hard conversations" of FASD and historical trauma. One Natural Helper explained:

When we are doing meetings and groups we have that general respect going around in circles, but also involving children because that's one of the biggest things that I always thought was frustrating was that governments or councils, they don't want to involve children or let children be around and I thought that was kind of a little strange because a lot of the decisions that we make affect our children. And that definitely is like a cultural emphasis for me because a lot of government councils would have their children or their wives, somebody in their family with them around. It's not like people around in a conference room with everyone quite and just adults. There are children everywhere and you know, there are spouses and partners involved in this too. It's not just us excluding other people from being in these meetings. Anybody can come. Anybody can be involved. And that's definitely something specific for Native communities 
because we definitely like people to jump in and we don't want to discourage anyone from being a part of this.

FGC members described how the status quo of government agencies excluding family members from meeting and events is culturally inappropriate for Native communities. Further, this quote reflects FGC members' emphasis on including family and Native community members of all ages reflects in FGC meetings and events. This reflects FGC members' view of Native traditional values as non-hierarchical and non-exclusive.

FGC members detailed the ways that County partners can act as allies in order to achieve successful Native-driven processes in the collaborative setting. Specifically, FGC members emphasized how County partners should focus on building authentic relationships based on respect and awareness rather trying to learn cultural appropriateness or cultural competency. One Native partner explained:

It's a conversation, not specifically around government appropriateness, but the necessity of government to understand the need of cultural relevant programing is important... Unless you're a Native organization I don't think that you should mingle in, I think you can be culturally responsive and have culturally competency but you can't-I can't even be culturally competent in the ways of my tribe, and I'm a tribal member. But I can be responsive and respectful to the fact that there is a cultural relevance for my tribe, and if I don't know those ways, I should ask the appropriate people about respectful behavior and protocol around those ways. And my ultimate desire would be that's the kind of relationships we have amongst our community. If you don't know, if you're not an expert or you haven't been rightfully trained in the ways of a certain program, institution, individual culture, then you should ask those respectful questions.

This Native partner clarifies the common theme that government agencies cannot create programing that is culturally appropriate or culturally safe without (a) including Native communities, (b) allowing Native experts to design and implement the cultural components of the innovative, and (c) asking for guidance from Native partners on how best on how best to act respectfully engage with Native communities and cultures. FGC 
members clarify that this is due in part to the complexities and diversity of Native cultures and communities. Moreover, since self-determination and Native engagement are such widely held Native values, processes can never be truly Native-driven if they do not include high degrees of Native representation. However, partners from government agencies can helpful by being respectful and responsive to Native culture and by creating conditions that enable Native participation in research and planning processes.

According to FGC members, County partners within the FGC did indeed go the distance to engage Native community within the FGC. One County partner described the FGC's approach of "attracting, recruiting, and retaining" Native engagement within the collaborative. She stated:

It's a platform or a venue...it's goal is to become a Native entity. So I hope that every Native voice feels safe and they feel safe to voice their opinions and that they have trust that their opinions are valued and are given weight in discussion or decision process and that we follow through with what we promised the community...Yeah, some examples of that is that we really we made an intentional effort to rotating meeting places so it's not always convenient for government staff. And offering incentives for the forums, like bus passes and all that. Like understanding community barriers to participation, like keeping community members from participating in these events.

The importance of governmental agencies honoring Native voices and self-determination within the FGC was a common theme described by FGC members. This quote also demonstrates that government agencies can help create space for Native voices and facilitate accessibility for Native community members through various measures.

Native partners recognized County partners desire to create space for Native community members and Native voices within the FGC, stating that County partners within the FGC were truly committed to the FGC's work. One Native partner gave an example of how County partners "fought tooth and nail" to secure gift cards, food, 
childcare, and transportation for community forum participants in an effort to make the forums more accessible and inclusive to members of the Portland Native community. She stated:

I mean that's like all stuff that the County rarely does, but the County workers knew the importance of it and fought like tooth and nail to make that happen. And I know the fighting that they did, that they never complain about. They never brought it to the bigger meetings or whatever but they would get in trouble because of the FGC. So that speaks volumes to what they're about and how committed they are to this project. They would get in trouble because of the FGC. But they were willing to do that, not to be a martyr, but they we're willing to do that because the people we're at the heart of the project.

This quote explains how County partners were willing to make personal sacrifices in order to facilitate Native inclusion within the FGC. It also points out that County partners were willing to work against the status quo of white-western domination within government agencies and advocate for the needs of the Portland Native community. Other Native partners often mirrored this theme stating that the level of dedication among County partners was rare within collaborative settings.

Moreover, FGC members described how government agencies were successful in acting as authentic allies by turning over design and implementation of the cultural and community engagement components to both Native partners and the Elders and Natural Helpers. For example, one Native partner described how her colleagues from the County had initially hoped to include public health curriculum into the trainings of the Elders and Natural Helpers, but had turned over the design and implementation of the trainings to Native partners. This demonstrated County partners' high level of trust in their Native colleagues. Similarly, one Natural Helper described how Native FGC members were 
successfully represented within the community engagement events hosted by the FGC.

She notes:

I think it is culturally appropriate because most people that are involved and most people that are going to be working with the community are Native or mostly Native. And what we're doing is programs that are designed for the community and the community is going to be able to look at us and see that we are not just being trained as robots to go and get information they won't want. We're actually listening to the community and giving feedback.

Native FGC members were successfully represented within the design and implementation of FGC, thus making the community forums both Native-driven and culturally appropriate. County partners likewise related how they attempted to "step back" and create space Native FGC members by turning over roles within the design and implementation of cultural and community engagement pieces of the trauma-informed process, including roles at the community forums, the Meaning Making Meetings, and the GONA.

FGC members described successes within the FGC's trauma-informed model based on a high degree of respectfulness and responsiveness from County partners. All Native partners and most Elders and Natural Helpers continually described County partners as "non-stereotypical" government employees and authentic allies. In order to become authentic allies, County partners engaged in training about historical trauma and Native community and culture that enabled them to adjust as necessary to Native feedback. One County partner explained her approach to acting respectful and responsive:

Because I think there's a difference between saying, "Yeah I totally support this, right?" and "I totally want to do it" and then going into a meeting and saying something that's offensive or getting critiqued on, "Well, that was a little weird, what you said" or "This isn't really working for us" and having the self-awareness 
and skill as a government employee to say, "Thanks for the feedback." Not like, "Well, that's not what I meant," right? Which it kind of comes down to antioppression work again, but for me that's a big part of the capacity building is helping government individuals, folks working in government, individuals and teams build skill, awareness, and knowledge of how to be a humble partner. How to approach partnership with humility and grace and kind of let go of this idea that, "I'm culturally competent," right? Because that's not what we're going for.

This quote reflects the ways in which County partners internalized the feedback from their Native colleagues and developed "self-awareness and skill" to engage in difficult conversations and address oppressive enabling ideologies, discrimination, and structural inequalities from their agencies. This level of respect from County partners towards their Native colleagues enabled responsiveness to suggestions of how to engage with the Portland Native community. It also explains the common themes of how County partners were willing to adjust language, methods, as well as organizational roles and goals to meet the needs of the Native community.

\section{Impacts of the FGC's Trauma-Informed Model on Native Health}

Throughout this chapter, FGC members describe how several characteristics of the FGC's trauma-informed model that have had positive impacts on the health of the Portland Native community. Common themes include engaging the Portland Native community in healing opportunities, creating a model for Native engagement in within research and planning process, and increasing governmental agencies capacity to collaborate and serve the Portland Native community. Increasing Portland Native community members' knowledge of historical trauma and substance-exposed pregnancies was another common theme, especially facilitating educational opportunities for Native youth during FGC meetings and events. FGC members also emphasized the trauma- 
informed process as supportive of the health and wellbeing of FGC members, especially the Elders and Natural Helpers. In this way, FGC members frequently describe how the FGC has created positive role models in ways that contribute to changing community norms.

Several FGC members describe how the trauma-informed model's holistic approach to promoting healing is necessary for addressing historical trauma and substance-exposed pregnancies within the Portland Native community. One Natural Helper explained:

It's going to be years before we see numbers [of substance-exposed pregnancies] go down, only because there's - not that we're not working hard, but there is just so much to work on. Like I said, this is just going to open doors to other issues, and we have a lot of them. I can speak for myself that I know that I have a lot of work to do. So as a community, to be able to do personal work and then find it within ourselves to come together and do the community work. Because first it goes self, families, and then community. Maybe not even particularly in that order, but it has got to start somewhere but I don't think that it's- how do I put it? I think it can happen; it's just going to be a lot of hard work on our part and on the community's part, their willingness to change and wanting to do something different. And there's a lot of mistrust I think within the community. As Native people too, we don't trust each other either, let alone the government. A lot of us don't even trust ourselves. I don't know, but I think what we're doing here is good. If anything, it has some positive impacts on us, as a people.

According to this Natural Helper, the FGC's impacts on Native health cannot easily be quantified into an immediate impact on the rates of substance-exposed pregnancies within the Portland Native community. Rather, the FGC is described as working hard on a holistic and long-term approach to making positive impacts on Portland Native community health by supporting multi-levels of Native American individuals, families, and communities. 
Impacts of the FGC's Trauma-Informed Models on Collaborative Partnerships

Building community capacity, Native representation, and Native-driven processes all contributed to strong relationships between County partners and Native FGC members. Many FGC partners emphasized how the trauma-informed process of partnership development built community capacity by "building trust one brick at a time" and helping to transform County partners into authentic allies within the Portland Native community. One Native partner stated:

The personal transformation that's occurring there has really been tremendous for me, how we're learning to step up and step back. Especially one of our County partners, her being a non-Native person, watching her grow-coming from a mainstream white majority population experience to several years of working intimately with us as Native people. She's getting it, and she's opening her heart and making space to be an authentic ally. And to watch that personal transformation with the people we are working with is progress in this work for me. And it's progress for me personally and spiritually, to aspire to be that kind of individual and so it's really kind of on a deep level.

Represented here is the common theme that County partners within the FGC were "likeminded and like-hearted". These qualities not only contributed to trust-building and strong partnerships but also to a sense of community and strong interpersonal relationships within the FGC.

Additionally, FGC members described some signs that the trauma-informed process was already creating impacts on governmental agencies outside the FGC. This way evidenced by outside governmental agencies' positive reception of the FGC's trauma-informed process at partnership meetings, by their participation in the GONA, and recognition of the FGC's trauma-informed model. Some FGC members described how the FGC's model of trauma-informed partnership development has increased hope that government agencies and Native organizations could work together to affect change 
in the Portland Native community.

\section{Conclusion}

Throughout this chapter, FGC members share their evaluations of the successful impacts of the FGC's trauma-informed process in terms of building community capacity, facilitating Native representation, and developing Native-driven processes. In this way, the FGC's trauma-informed process can be seen as positively impacting partnerships between government relationships and the Portland Native community within the FGC.

This is accomplished in three way: (a) by successfully intervening on the historical trauma process within the context of the FGC, (b) by supporting Native protective factors and thereby contributing to positive Native health outcomes, and (c) by building trust within partnerships between government agencies and the Portland Native community. In order to address the limitations of the trauma-informed model, the next chapter covers FGC members' evaluations of the barriers the FGC has faced throughout their research and planning process. The next chapter also details how FGC members have attempted to overcome these challenges. 


\section{Chapter 8: Findings on the Barriers to the FGC's Trauma-Informed Model}

\section{Introduction}

Despite the fact that most FGC members recognized a significant shift within the

collaborative partnerships between government agencies and the Portland Native

community within the FGC, all FGC members discussed how structural inequalities

continue to create unequal relationships between government agencies and Native

communities at large. One County partner stated:

But the FGC and what we're doing, particularly in terms of organizational and systems change is really outside of the box for how most government organizations think about partnership, even organizations that understand traumainformed care. The idea of trauma-informed collaboration is a lot to wrap their heads around. So I think that we are really just at the initial seeding stage where maybe we're not even planting the seeds yet. We're really just preparing the ground to plant the seeds, right? Where this new form of collaboration and this cultural shift can really take root and grow and really become a thriving ecosystem, if you will? Where it's rich with trust, it's rich with partnership, it's rich with collaboration and communication and really it's nurtured by those relationships and by multiple people and multiple inputs, right? We're not there yet, although I think there are rays of hope...it hasn't reached critical mass, yet. And I think we are a ways away from that.

This demonstrates the common theme that since the initial shift to a trauma-informed model, the FGC has by no means eliminated the structural inequalities between government agencies and Native communities. Further, FGC members highlight how these structural inequalities continue the systemic perpetuation of historical trauma by government agencies in Native communities. In this way, the FGC has continually come up against the persistent structural inequalities of the historical trauma process. Many FGC members describe how the FGC has begun the collaborative process of addressing and healing from the systemic perpetuation of historical trauma within the specific 
context of the FGC's trauma-informed model. However, nearly all FGC members mirror the theme that there is still a long way to go.

Throughout participant observation and interviews, FGC members described at length the barriers faced by the FGC throughout their trauma-informed research and planning process. Some FGC members explained how these barriers limited the effectiveness of the FGC's trauma-informed model. Other FGC members saw how these barriers signified challenges that the FGC has sought — or is presently seeking — to overcome. The latter optimistic description of barriers accentuates the FGC's traumainformed process as continually shifting to meet the natural challenges of collaborative research and planning. One Natural Helper explained how the FGC's trauma-informed process is like a river current, with the highs and lows of the upstream tide representing the various barriers and solutions experienced within the FGC's model. Whether FGC members felt skeptical or hopeful about the barriers experienced by the FGC, most FGC members acknowledged the existence of barriers to the FGC's trauma-informed process.

This chapter details FGC members' evaluations of the barriers to the FGC's trauma-informed research and planning process. These barriers include government agencies' continued role in the systemic transmission of historical trauma within Native communities. To this end, the following sections explain FGC members' perspectives on how governmental structural inequalities of disinvestment into Native communities, Native exclusion, and reliance white-western dominated processes have created persistent obstacles for the FGC. This chapter also includes FGC members' discussions of how limiting factors within the Portland Native community have impacted the successfulness of the FGC's trauma-informed model. Exploration of the barriers to the FGC's trauma- 
informed process is essential for understanding the relationships between government agencies and the Portland Native community within the FGC.

\section{Continued Government Disinvestment and the Impact on the FGC}

FGC members discussed how government disinvestment into Native communities continues to act as a structural inequality that perpetuates historical trauma and creates barriers within the FGC. FGC members frequently described the trauma-informed process as incredibly successful despite the fact that the FGC operates on an exceptionally small amount of funding. FGC members explain that this is due to a high level of personal investment from all FGC members, including numerous hours of volunteered time and pooling of resources to provide for culturally appropriate necessities such as childcare, food, transportation and other Native-specific needs.

Nevertheless, FGC members detailed how funding is still needed for supporting staff from government agencies and Native organizations alike. FGC members also often described how the FGC needs funding to support the work of Elders and Natural Helpers through funding. Elders and Natural Helpers were often asked to volunteer their time within the FGC, despite the fact that as a group they are more likely to experience financial constraints to participation within the FGC. One Native partner revealed:

From my perspective working in these systems, I think the amount of funding indicates the priority that a project carries within a system like Multnomah County. And because we don't have dedicated funding and it's a struggle to scrape together the little bits that we do have, I don't see importance.

Reflected here is the common theme that the persistent lack of dedicated funding from the County represents a continuation of disinvestment into Native communities. The 
quote also points out that the FGC has struggled "to scrape together the little bits" in order to successfully design and implement the trauma-informed process, thus outlining how FGC members have been required to make personal and organizational sacrifices in order to accomplish their goals within the FGC.

FGC members describe the high level of commitment from individual County partners within the FGC and receptiveness of some government agencies during partnership outreach meetings. At the same time, the FGC struggled against government disinvestment into Native partnerships within the FGC. This was demonstrated by the fact that a number of governmental partners dropped dramatically over the course of the FGC's trauma-informed process. Initially, the FGC was comprised of a majority of County partners. During my year of participant observation the number of County partners with dedicated work time toward the FGC dropped in half, from four to two. While a lot of these transitions were based on personal and professional transitions experienced by governmental partners, government agencies did not replace the staff within the collaborative after they moved on from their work. One County partner explained:

There's no way that I can be at all the meetings and its disconcerting to me because we don't, not everybody has the 10,000 foot view and so I feel like pieces of information get lost, people get lost, and that because were kind of at capacity. I'm at capacity, were not always living up the values in the trauma-informed process, and that's a big concern for me... What I'm concerned about are the pressures on me, that I feel like now that other County partners are gone that I'm the only person doing this work. If I get sick, if I have a family emergency, if I go on any extended vacation, shit falls apart. And that is deeply concerning to me, that's another way I think about sustainability. When I think about, it's not sustainable if there are such fragile points of connection that keep the momentum going, that if one of those pieces comes apart things start to unravel...I don't think we're safe yet. Let me just say this too, if I left the County tomorrow there would be no one else to carry out this work, and that is not okay with me. 
This quote exemplifies the exceptionally high demand placed on County partners within the FGC who often described themselves "at" or "above" capacity in terms of their ability to dedicate time and energy into the FGC. Many FGC members described a need for a full-time County staff member who could dedicate all their work hours to the administrative functions of the FGC. Similarly, Native partners regularly described how their work within FGC sometimes represented a "thorn" due to the limited funding and the necessity for volunteered time in order to fulfill their roles within the FGC. The fact that so many FGC members have retained active participation in the FGC despite this enormous obstacle speaks to remarkably high level of personal investment by FGC members into their work with the FGC.

Additionally, FGC members agreed on the need for funding of organizational infrastructure and so that the FGC can create an intervention based on the feedback from the community forums, the Meaning Making Meeting, and the GONA. One County partner detailed:

I mean we're at a point where we really need to be able to get that funding to support long-term involvement, you know because we can't do the interventions we need to do without the funding. And so it's just critical. I mean we've been doing great at the planning process but we need to go into the doing process, and if we cannot do, then we're not going to maintain the support. We've developed the hope. We've got to be able to get the funding so the doing can happen, because the doing can't happen without funding. It's just critical to be able to support the interventions with money... but we're going to need to be able to get some funding from different sources all so to be able to put in some infrastructure.

FGC members frequently shared the theme that the FGC has been able to successfully build their high degree of engagement from the Portland Native community by promising to address substance-exposed pregnancies and historical trauma within the Portland 
Native community. If the FGC is not able to deliver on their commitments to the Portland Native community, then the collaborative will likely create another breach of trust in the relationships between government agencies and the Portland Native community.

Likewise, this lack of funding from government agencies was described as especially problematic considering the continued lack of governmental financial support for Nativespecific programing within the Portland area, including extremely limited resources for Native families experiencing FASD.

\section{Continued Governmental Exclusion and the Impact on the FGC}

FGC members also described how the structural inequality of Native exclusion within government agencies continues the perpetuation of historical trauma and produces barriers to the FGC's trauma-informed model. For FGC members this was evidenced by certain governmental agencies not listening during partnership presentations and government agencies who just "didn't get" the type of support the FGC was requesting.

One Natural Helper stated:

I know that the FGC went to go present at one government agency and they didn't get it. And it's like they were just blanking out. They were busy on their iPad or they were just busy elsewhere. Until finally someone from the agency put their hand up to ask a question and it was, "How do we make referrals to you?" Totally missed the point! And she had to say, "We don't provide that type of service." Where have you been for the last hour? No, she didn't say that, but it was like good grief! Yeah, they were just over their heads. And they just were not present, so it's hard to say what can happen there.

This Natural Helper illustrated the ways in which government agencies continued to exclude Native voices by not listening during partnership meetings. Similarly, FGC members described how some governmental agencies lacked follow through after 
providing "lip-service" support for the FGC during partnership meetings, thus perpetuating the exclusion of Native community from governmental agencies.

Moreover, while most Native partners and Elders and Natural Helpers interviewed believed that the Portland Native community was strongly represented within the FGC, a few Native FGC members reported feelings of disengagement within the FGC. Eighteen Elders and Natural Helpers initially signed up to participate in the trainings, the community forums and the Meaning Making Meetings. After the Meaning Making Meetings, participation dropped off and the FGC lost four Elders and Natural Helpers. FGC members often explained this as a normal retention loss. This small number of retention loss was also understandable considering how the roles of Elders and Natural Helpers were transitioning from community members receiving stipends to aid in technical assistance and implementation in the FGC's research, to community members volunteering their time to engaged in the trauma-informed research and planning process. During that time an additional five Elders and Natural Helpers left the FGC for various reasons. A year after the initial trainings, approximately nine Elders and Natural Helpers continued to be involved with the FGC despite the lack of funding for their positions. Many FGC members described the FGC's retention rate of Elders and Natural Helpers as a huge success, especially considering the limited budget and the normal fluctuation of community participation on a semi-volunteer basis. At the same time, some FGC members discussed barriers of Native exclusion that contributed to the retention loss experienced by the FGC. One explanation for Native disengagement within the FGC included unclear roles for Elders and Natural Helpers, who wanted to be more involved 
than they were. One Native partner also echoed this theme, after facing difficulty

integrating into the FGC's already established organizational partnerships.

FGC members also discussed Elder and Natural Helper loss of retention based on the controversial subject matter of historical trauma and FASD. FGC members explained how these topics sometimes trigger trauma and create conflict. While FGC partners participated in a long educational and partnership building period where they addressed historical trauma explicitly and worked to resolve conflict, Elders and Natural Helpers were not present during this portion of the FGC. One Native partner explained conflict among the Elders and Natural Helpers on the FGC's Facebook page:

And Elders and Natural Helpers are a big part of that and have come full circle about that, and are kind of having some of the growing pains that we had in the very beginning of: How do you talk about the hard stuff? How do you talk about walking your walk and walking your talk? Because that's hard, if you're not used to it, it's really hard. And that includes online: it's an electronic signature you can never take back. Think about that when you hit that post.

This explanation was frequently cited when describing the "growing pains" and "lateral oppression" experienced within the group of Elders and Natural Helpers. This quote also helps to explain some of the loss of retention due to internal conflict within the Elders and Natural Helpers.

Another common explanation for Native disengagement came from a small number of Elders and Natural Helpers who saw barriers between the inclusion of Elders and Natural Helpers voices within the FGC's planning process. One Natural Helper explained how she viewed hierarchies within the FGC that limited Native community members in their role as Elders and Natural Helpers:

I think that most of the Elders and Natural Helpers are Native. They don't really have any decision making power, they're not the ones who control the purse 
strings or control the direction or anything, and I do know that, I think I'm not sure. This sounds crazy, one County partner will say, "Well, I want your input and I want your help and we want you to be a part of this." But then I don't see where they actually hear what we're saying... And as an Elder and Natural Helper, we're asked to come to the meetings, and I know we make comments, but I don't know that any of our ideas are ever put into place... So that's a little bit frustrating so then you tend to not want to participate and it's gotten to the point where it's them and us. And that's not a good thing, you know? It's like they're having their meeting on FGC and we'll have our meeting on FGC, you know? So we have our meetings on Wednesday evenings, with the Elders and Natural Helpers, and they have their meetings during the day...I don't know if it's growing apart or if it just needs to, maybe we have different kinds of issues, but I know what we're doing on our Wednesdays is we are informing each other about different things that are going on in the community that I'm sure FGC really doesn't have any stake in.

As demonstrated in this quote, a few Elders and Natural Helpers' view the their participation within the FGC as in on a second tier of importance within the FGC. This theme was more common amongst Elders and Natural Helpers who were employed, had experience working within an official capacity in Native communities, and who could have easily been considered Native partners representing Native organizations.

Coupled with this belief is the perception that County partners in administrative capacities are not completely sharing power with Native community members and are "controlling the purse strings" of the Future Generations Collaborative. Most FGC members see County partners as authentic allies. However, many Elders and Natural Helpers were unaware of the partnership development under which the roles for the County and Native organizations were agreed upon collectively through the traumainformed process. This suggests some barriers to Native inclusion within the partnership development component of the FGC's trauma-informed model. Additionally, the fact that most FGC partners report being "at" or "above" capacity while some Elders and Natural 
Helpers feel that they do not have a role within the FGC also lends credence to the notion that work within the FGC is not always distributed effectively within the FGC.

\section{Continued White-Western Dominated Processes and the Impact on the FGC}

FGC members discussed how structural inequalities of white-western dominated processes within government agencies continued to create barriers within the FGC as well as perpetuate historical trauma within Native communities. FGC members provided examples of government agencies that were not receptive to the FGC's trauma-informed model during partnership meetings. FGC members said that some government agencies have a long way to go before they truly understand trauma-informed research and planning. One County partner explained:

Most of these entities - understand trauma-informed clinical interventions, they don't understand trauma based planning processes. And that's very different because that has to take equal partnership and if they don't have equal partnership they're going to get the same old results that they have in the past because they're going at it from a very paternalistic way of planning... So that really has a lot to do with, you know that group and how open they are to understanding innovative approaches. You know? So people are on a huge spectrum. Those that don't even want to hear about it, it's you know...I used to have a supervisor at one government agency that would talk about, "Oh, there putting in a new process. But it's just the same old stuff with different words. We just do what we're going to do." And so if you've got that mind set you're not going to be able to move into new processes because it's like, "Hey I'm going to put a different word. I'm going to spin it a little. I'm going to make it look like we're involved" but you really don't. Some of those it takes a long, long time to change that kind of behavior. So that's just, having to have multiple meetings. Maybe putting pressure from outside entities on them. It's going to take more than just a partnership meeting for those entities.

This quote illustrates how the structural inequality of reliance on white-western dominated processes is deeply engrained in many government agencies. Therefore, these government agencies are not willing to change in order to become authentic partners 
within processes they are unfamiliar with, including trauma-informed research and planning. Another County partner agreed that many government agencies are unlikely to change their processes voluntarily without pressure from the Portland Native community.

FGC members also described how bureaucracy within government agencies has continued to represent the structural inequality of white-western dominated processes and created large barriers to the FGC's trauma-informed process. For example, several FGC members described how County bureaucracy created barriers for the FGC through their contracting rules and regulations. One Native partner explained:

And so, from my perspective with the contracting and stuff, in the very beginning it was frustrating with the County. And again I'm getting back to the whole money thing, and the amount of work we needed to do just to get the contract signed, and the amount of money that we had to spend to meet their requirements, that was frustrating and I think a lot of people would have just thrown in the towel and said, "Screw you guys, this is too crazy." But we didn't we hung in there, again because we saw the bigger picture, right? And we know because of other experiences that you just have to jump through these hoops sometimes but the people are always why we jump through the hoops.

Bureaucratic processes create "hoops to jump through" that are incompatible with Native driven processes and continue to create barriers for Native organizations within the FGC. At the same time, FGC members describe how the trauma-informed process helps to make these barriers tolerable by helping to challenge white-western dominated processes, by keeping the Portland Native community at the heart of the project, and through the support of FGC County partners who advocate on the their Native colleagues' behalf.

Furthermore, FGC members discussed the ways in which white-western dominated processes have created barriers during FGC meetings that are sometimes still described as linear and outcomes-oriented. One Native partner explained how the absence 
of County partners' linear and outcome-oriented processes in one planning meeting yielded equally effective results:

So we didn't have a written agenda, whatever, whatever all this kind of stuff. But look at product at the end, it was the same as if we had all that stuff in place, you know what I mean? And there we joked and laughed about it. And some people have come a long way in that department, of understanding well there's one way, that's one way to do it, and not just in meetings but in everything, right? And there's always another way, and there are always ten more ways between those ways and so I think as any group goes a long we learn everybody's style and personalities and all that kind of stuff.

This quote highlights how the linear, rigid, and outcome-oriented processes of County partners have increased in flexibility over the course of the FGC's trauma-informed process, yet retained some of their hold over the methods used during FGC planning meetings. FGC members described how this barrier has in many ways been successfully overcome by encouraging County partners to relax and "trust the process" and by continually advocating for greater inclusion of Native processes within FGC meetings.

Throughout participant observation, some FGC members described how uses of language within the FGC sometimes privilege the white-western dominated language of public health over the languages of Native communities. For example, County partners created logic models to describe the FGC's work and the trauma-informed process. These models were described as non-representative of Native ways of knowing and were sometimes challenged during FGC meetings. Similarly, one Native partner explained:

I think integrating these different learning and teaching styles and these different teaching approaches and stuff that the County offers training in, are really in my opinion Native-specific teaching tools and models. So even though some people at the County may think they're using, you know popular education approaches, I mean really it's Native approaches that they're talking about. That's how I see it. It's like connecting the head and the heart...I think County partners are still getting there, and vice versa, we're trying to better understand where they coming 
from and understand all that kind of stuff. So that goes both ways, I think that has been good.

This quote explains how County partners' may be discussing similar approaches to public health that are common within Native-driven processes. However, their use of public health language still privileges white-western dominated processes. Importantly, this Native partner also points out how reciprocal learning is taking place in ways that undermine white-western dominated processes within the FGC. This reciprocal learning is based on a high level of respect between County partners and the Portland Native community within the FGC.

Barriers to the FGC's Trauma-Informed Process from the Portland Native Community

While the systemic perpetuation of historical trauma has been largely held responsible for the barriers faced by the FGC, FGC members also detailed how barriers within the Native community have limited the widespread involvement of the Portland Native community. First of all, many FGC members describe the ways in which the Portland Native community is not a singular and cohesive group. Therefore, while engagement is high within certain portions of the Portland Native community, other groups are still unengaged in the FGC's work. One Native partner stated the significance of the FGC's lack of formal partnership with the Confederated Tribes of Grand Ronde, whose ceded lands include the Multnomah County area:

There is no formal partnership between Grand Ronde and the FGC. And there's like over 600 Grand Ronde tribal members living in Portland, so they are stakeholders definitely but they are not involved in the decision-making. Maybe that's beginning and that takes time anyway. There's a lot of Native people who aren't being represented at the table. I mean there's Grand Ronde, like I said before and there's all the other federally recognized tribes that are in Oregon. I 
think Siletz has people there, but I think that might be it for the Oregon Tribes. So there's work to be done there. The urban community is so different than the tribal communities in Oregon, they have different problems and different needs and different cultures...

A common theme among FGC members was how the FGC has missed building bridges with local tribes. Therefore, the FGC is missing the voices and the needs of important stakeholders within the Portland Native community. This quote also highlights a common theme within the FGC of the importance of recognizing the diversity within the Portland Native community and the need for the FGC to seek greater levels of Native American inclusion.

While the FGC may be explicitly focused on serving the urban Native population within Portland, many urban Portland Native community members remain strong ties to their tribe and therefore have specific cultural needs that are not addressed by a more broad-based intertribal approach. One Natural Helper stated:

A lot of time it doesn't seem like there's anything they can do and problems seem overwhelming, especially in our community. I know that there's a lot that goes on especially in the reservations. When people move to the city they get caught up in the concrete jungle type of things and there's other issues. Some of them are the same, but they look different. They wear different clothes and they have a different appearance and they even have a different feel sometimes, and sometimes it's just a relief to get away from that stuff. And then you get so caught up in other daily living that you don't, it's not easy to see an answer, or a way to do something that would benefit our people on a cultural level, or on as deep of a level that would not only help our urban Indian inner city community, but also reach out to the reservations and do anything there because it's so far away and it's like a whole other world sometimes. Especially for those of us who are from tribes that aren't here in Oregon, a lot of times it just seems like a whole different world. It's way over there, it's a different planet. And things are way different, and how could I possibly do something that would help our people here living this life, with technology?

Represented here is the common theme that the FGC cannot truly address substanceexposed pregnancies and historical trauma within the Portland Native community without 
addressing these issues on reservations. This is due to the fact that "urban" Native

community members retain a wide-spectrum of ties to their reservations. This theme was reflected not only by FGC members during interviews and participant observation at meetings, but also during the community forums where Portland Native community members were asked about how best the FGC should approach substance-exposed pregnancies and historical trauma.

FGC members also described how Portland Native community engagement has been limited by the fact that not all Portland Native community members are open to discussing historical trauma and FASD. One Natural Helper recalled her interaction with a Native Elder at one of the community forums. She stated:

I know some of the Elders were all for talking about historical trauma and some Elders were kind of bothered by it because it was like, "This is all we talk about, but nothing is ever being done." So I know there was this Elder lady and she stepped out of the room when I was a greeter and I was like, "Oh, you're not going to go in there?" And she was like, "Oh they're just talking about same old, same old. Historical trauma, yada, yada, yada. We got to move on from that. Why are people still bringing it up?" And I told her, "Sometimes it affects people differently, others actually want to talk about it. Just like I bet you they're going off about it in there." She's like, "Oh yeah, they are." I was like, "Yeah, see and maybe others like you just want to stop talking about it and want to keep moving forward, right?" She said, "Yes. And that's why I'm out here eating my food with you." I was like, "Okay." So I wasn't offended by her not being there. I totally understood where she was coming from, and like I told her, everyone just has different opinions.

As stated by this Natural Helper, some Portland Native community members are uncomfortable or frustrated with the discussion of historical trauma. Importantly, FGC members pointed out how the high level of engagement of Portland Native community members suggested that the FGC could still contribute to the healing of historical trauma even if the concept did not yet resonate with all Portland Native community members. 
Similarly, FGC members discussed multiple misperceptions of the FGC that stood in the way of Native community engagement. Many FGC members commented on the fact that some Portland Native community members saw the FGC as a "recovery group" based on how healing from historical trauma and FASD were framed by the collaborative. FGC members stated how this tended to exclude Portland Native community members who drink alcohol and use drugs. FGC members also stated how some Portland Native community members' perceived the FGC as a "pregnant ladies group". This discouraged participation in the FGC by Native American males and some Native American women who were not of reproductive age.

Another barrier described by FGC members was Native American mistrust of programs and partnerships to address Native issues. Native American mistrust was described as a product of the historical trauma process, and specifically the long legacy of lack of follow through and "nothing ever being done". This theme was described at length when FGC members expressed concerns about the delays in the GONA. One Natural Helper commented on how some Portland Native community members were skeptical about the FGC's effectiveness based on the fact that the GONA had been pushed back multiple times over a six month period. She stated:

I've been really trying my hardest to continue to talk to the community members about the program and telling them what kind of progress we're making. And trying to promote the GONA conference and make sure people still know that the FGC is still doing work and still trying to put together this conference because its been so long since the group forums and the Meaning Making Meeting. A lot of people still haven't heard about it and a lot of people kind of forgot that we had done that in a lot of ways. So I've been really kind of just trying to keep the community, and remind the community that we are still doing this and we are still trying to take that information and use it. 
A few FGC members mirrored this theme that some Portland Native community members felt skeptical about the effectiveness of the FGC due to a gap in time between the Meaning Making Meetings and the GONA. FGC members discussed how this was resolved once the GONA was actually held, as evidenced by the large turn out at the event. Now that the GONA is over, FGC members continue to describe the need for continued engagement with the Portland Native community and implementation of Portland Native community feedback from the GONA in order to maintain active engagement.

\section{Conclusion}

While most FGC members reflected the optimistic view that the FGC has made long-term and holistic impacts on Native health in Portland, a few FGC members report that the positive impacts on Native health "remains to be seen". Most FGC members report that the trauma-informed process created strong relationships and trust within the relationships between County partners and Native FGC members within the FGC. At the same time, this trust was not completely shared by all FGC members. FGC members also described how this trust was limited to the partnerships between government agencies and the Portland Native within the FGC and continued to describe trust as "non-existent" between government agencies and the Portland Native community at large. FGC members also clearly stated that just because trust was built within the FGC collaborative partnerships, does not mean that all Native FGC members now trust government agencies outside the FGC. Similarly, FGC members reported how the success of the FGC's 
trauma-informed process has not wiped away greater governmental agencies' mistrust of the Portland Native community within the FGC.

This chapter demonstrates FGC members' evaluations of how government agencies are continuing the systemic transmission of historical trauma both within Native communities inside and outside the context of the FGC. Therefore, the on-going process of historical trauma continues to create obstacles for the FGC's trauma-informed research and planning. Analysis from this chapter also supports the continued discussion of how historical trauma and the FGC's trauma-informed model affect the relationships between government agencies and the Portland Native community within the FGC. 


\section{Chapter 9: Discussion}

\section{Introduction}

The Future Generations Collaborative (FGC) provides a unique case study for examining the partnerships between government agencies and the Portland Native community. Prior to the FGC, the active presence of the historical trauma process prevented these types of collaborative partnerships. Additionally, historical trauma has contributed to deep health inequalities experienced by the Portland Native community, including the high rate of Fetal Alcohol Spectrum Disorder (FASD). Despite the large barriers created by historical trauma, representatives from the government agencies within Multnomah County and as several Native-serving organizations were able to come together and develop a trauma-informed model of community-based participatory research and planning.

The FGC's trauma-informed model was able to successfully shift County partners' outcome oriented approach to addressing substance-exposed pregnancies into a holistic healing process that serves to promote healthy pregnancies and healing form historical trauma within the Portland Native community. Thus the FGC's trauma-informed process represents a significant shift in the relationships between government agencies and the Portland Native community. In order to understand how historical trauma and the FGC's trauma-informed process affect the relationships between government agencies and the Portland Native community within the FGC, this study focuses on three central research questions:

1. How do FGC member's describe the government's role in creating and sustaining historical trauma in Native communities? 
2. How does historical trauma affect the relationship between government agencies and the Portland Native community within the FGC?

3. How does the FGC's trauma-informed research and planning process affect the relationships between government agencies and the Portland Native community within the FGC?

The next three sections discuss how the findings from this study address each of these research questions. These sections are followed by detailed descriptions of this case study's implications.

\section{The Government's Role in Creating and Sustaining Historical Trauma}

Historical trauma has deeply impacted the relationships between government agencies and Native communities. FGC members describe how government agencies have taken an active role in creating the initial incidence of historical trauma through various colonial projects. These periods of historical trauma have had devastating impacts on the first generations of Native Americans who initial experience colonization.

Historical trauma is then passed down intergenerationally through collective memory and cumulative disadvantage within Native communities. This process has contributed to major disparities in terms of the social determinants of health and therefore explains the extreme health inequalities faced by Native communities today. Specifically, FGC members describe the impacts of the historical trauma process on high rates of FASD and other substance-exposed pregnancies within the Portland Native community. This process also contributes to the immense mistrust in the relationships between government agencies and Native communities.

The government also sustains historical trauma through a complex process that perpetuates historical trauma systematically over the generations. The systemic 
transmission of historical trauma is characterized by a vicious cycle of contemporary traumas within governmental agencies. This vicious cycle is maintained by three interlocking mechanisms, including structural inequalities, enabling ideologies, and discrimination. All three of these mechanisms serve to mutually reinforce each other over time so that historical trauma is continually compounded and passed on to generations of Native Americans who come in contact with government agencies.

FGC members described the historical trauma process in detail through the discussion of three primary structural inequalities within government agencies. The structural inequalities include: (a) government disinvestment into Native communities, (b) Native exclusion from government agencies, and (c) governmental reliance on whitewestern dominated processes. The systemic transmission of historical trauma by government agencies serves to deepen the inequalities experienced by Native communities. Additionally, the systemic transmission of historical trauma exasperates the high levels of mistrust between government agencies and Native communities.

Native communities maintain an abundance of protective factors that help buffer against the affects of historical trauma. These protective factors included Native community strengths and resources on four levels, including: Native American individual, family, community, and culture protective factors. Furthermore, Native communities have always resisted the government's initial creation of historical trauma through colonial projects as well as the perpetuation of historical trauma through structural inequalities, enabling ideologies, and discrimination. Both Native protective factors and Native resistance have helped promote resilient outcomes in Native communities as well as contributed to healthier relationships between government agencies and Native 
communities. At the same time, Native resistance and protective factors are not yet at the point where they are able to completely mediate the affects of historical trauma (see Figure 4, p. 90).

\section{Historical Trauma Impacts on Collaborative Partnerships within the FGC}

Government agencies are responsible for creating historical trauma through various colonial projects across the United States. Both local and national incidences of historical trauma have created profound impacts on the relationships between government agencies and Native communities in Portland, OR. Furthermore, government agencies have continued the systemic perpetuation of historical trauma in Native communities in general through the vicious cycle of contemporary traumas. This process deepens historical trauma for every generation of Native Americans that come into contact with structural inequalities within government agencies. Thus the historical trauma process among Native Americans generally helps to contextualize massive Native health inequalities in the Portland area — including high rates of FASD — as well as high levels of mistrust between government agencies and Native communities in Portland.

FGC members also describe how historical trauma specific to the Portland area has occurred in ways that have compounded the health inequalities and governmental mistrust within the Portland Native community. For example, the Multnomah County Health Department was responsible for contributing to health inequalities and mistrust based on the historically traumatic period of sterilizations in Oregon. Historical trauma was also perpetuated systemically by the government agencies in their initial approach to addressing substance-exposed pregnancies in the Portland Native community, prior to the 
formation of the FGC. This approach was characterized by the same structural inequalities of disinvestment into Native communities, Native exclusion, and whitewestern domination.

The mistrust created in this initial approach by government agencies almost halted collaborative partnerships before the FGC got started. However, the FGC was able to confront the historical trauma process and it's impacts on Native health and relationships between government agencies and the Portland Native community by developing a trauma-informed process for research and planning. Once the trauma-informed model was developed, the historical trauma process continued to affect relationships between government agencies and Native communities within the FGC through the structural inequalities described above. These structural inequalities were described as embedded in the relationships between government agencies and the Portland Native community within the FGC. Additionally, structural inequalities were imposed upon the FGC thorough external pressures based on the endemic nature of the historical trauma process within governmental agencies at large (see Figure 4, p. 90).

\section{The Trauma-Informed Model's Impacts on Collaborative Partnerships within the FGC}

Several factors contributed to the formation of the FGC and the development of a trauma-informed model for research and planning. First, Native partners skillfully resisted the County's initial approach, by calling County partners to address their department's role in creating and sustaining historical trauma. Secondly, County partners were willing to (a) acknowledge their department's role within the systemic transmission of historical trauma, (b) make adjustments to address the structural inequalities inherit in 
their approach and (c) support Native protective factors. These factors combined to create a significant shift in the relationships between government agencies and the Portland Native community within the FGC.

The FGC began anew, grounded in Native partner's expertise of the government's role in systemically perpetuating historical trauma. Native partner's expertise also informed the development of an innovative model for community-based participatory research and planning to promote healthy pregnancies and healing from historical trauma within the Portland Native community. The trauma-informed model sought to accomplish these goals by engaging the Portland Native community and supporting Native protective factors that promote resilience to historical trauma. Moreover, the FGC's model also focused on healing the relationship between government agencies and the Portland Native community through trust-building and partnership development. In order to accomplish these goals, the FGC adapted several components of community-based participatory research methodologies to fit their trauma-informed approach.

The trauma-informed process made significant headway at reducing the affect of the structural inequalities within the FGC. This was accomplished by building community capacity, by creating space for Native representation, and by relying on Native-driven processes. These three components of the trauma-informed process created strong partnerships, characterized by a high degree of trust within relationships between government agencies and the Portland Native community within the FGC. This trust was strongest among FGC partners who experienced the partnership development phase of the trauma-informed process as well as Elders and Natural Helpers who maintained very active roles within the FGC. The FGC was also successful in terms of achieving an 
unprecedented level of engagement from the Portland Native community in a project that included government agencies.

At the same time, the ongoing historical trauma process continued to create barriers for the FGC. In response to these barriers, the FGC continually adapted their trauma-informed process in order to confront the systemic transmission of historical trauma that persisted both internally and externally of the FGC. In that way, the traumainformed research and planning process represented a healing process that continually shifted as necessary in order to address the needs of the Portland Native community at a deeper and deeper level.

The FGC's trauma-informed process can be seen as an effective tool for Nativedriven research and planning and for promoting healing from historical trauma. FGC members expect that this approach is the most effective approach to reducing substanceexposed pregnancies in the Portland Native community over the long term. The FGC's trauma-informed model also serves as an effective intervention on the relationships between government agencies and the Portland Native community through cultivating healing from historical trauma as well as making systematic changes to reduce the perpetuation of historical trauma. In all of these ways, the FGC was able to create profound affects on government relationships with Native communities within the FGC.

County partners, Native partners, and Elders and Natural Helpers all maintained roles within the FGC that were integral to the FGC's success. The wisdom and expertise of Native partners was absolutely essential in order to effectively resist the systemic perpetuation of historical trauma and to develop the FGC's trauma-informed process for research and planning. By taking the lead in partnership development, Native partners 
were able to successfully provide a transformative process in which County partners learned how to become authentic allies to the Portland Native community. Once County partners became authentic allies, they were able to contribute to the FGC by (a) acknowledging their departments role in creating and sustaining historical trauma, (b) making adjustments as necessary to address the systemic transmission of historical trauma, and (c) provide resources and advocacy to support Native protective factors.

Both Native partners and Elders and Natural Helpers were responsible for the high level of engagement of the Portland Native community. As Portland Native community members themselves, Native Partners and Elders and Natural Helpers were able to provide indispensible insight into the trauma-informed process as it evolved. Elders and Natural Helpers' growth and transformation were described as building the FGC's credibility within both governmental agencies and the Portland Native community. Elders and Natural Helpers also increased their capacity to serve the Portland Native community through participation within the FGC's trauma-informed process of research and planning. By participating in healing and professional development opportunities, Elders and Natural Helpers became positive role models who were empowered to advocate on behalf of the Portland Native community and influence Portland Native community norms.

The success of the FGC's trauma-informed model was somewhat limited by the deeply engrained nature of the historical trauma process within government agencies both internal and external of the FGC. The structural inequalities that systemically reinforce historical trauma are still widespread. This contributes to barriers that impeded upon FGC's efforts to build community capacity, create space for Native representation, and 
engage in Native-driven processes. Obstacles within the Portland Native community also affected the success of the FGC's trauma-informed research and planning model. However, with significant headway being made within the FGC, most FGC members were hopeful that the trauma-informed process could continue to work to address historical trauma in the relationships between government agencies and Native communities within the FGC and more broadly (see Figure 5, p. 105).

\section{Implications for Theorizing Historical Trauma}

Over the last twenty years, Native American scholars have made huge strides in developing theoretical frameworks for understanding historical trauma within Native communities (Fast and Collin-Vézina, 2010). This study makes a valuable contribution to the historical trauma literature by studying unique phenomenon within the relationships between government agencies and the Portland Native community. The context of Portland, OR is especially significant considering the county's specific colonial history and disproportionately high rates of health inequalities (Curry-Stevens et al. 2011). This research also adds to limited body of work on historical trauma in urban Native communities. Focus on the collaborative partnerships between government agencies and urban Native communities is significant considering how urban Native communities lack the legal support for self-determination, tribal autonomy, and sovereignty (Beck 2002).

Further, historical trauma scholars have called on researchers to address some gaps in the literature in regards to the social causes of historical trauma (Evans-Campbell 2008; Sotero 2006). FGC members describe a complex process in which government agencies create and sustain historical trauma in ways that are unique within the historical 
trauma literature. Native Americans within the FGC developed this expertise about the government's role in perpetuating historical trauma, suggesting that the systemic transmission of historical trauma described throughout this thesis is not new to indigenous epistemologies.

Nevertheless, academic writing focusing on how government agencies continue to perpetuate historical trauma is extremely limited. This is especially true within the field of sociology, where theory on historical trauma in Native communities is negligible. By bringing the theoretical insight of tribal critical race theory and Myrdal's (1962) vicious cycle together with the empirical findings from this case study, this research adds a sociological perspective to tease out the specific ways historical trauma is systemically perpetuated by government agencies. Academic research is important for increasing education around historical trauma as well as holding the government accountable for their role in historical trauma. More research should be done to explore Native perspectives on the role of government agencies in creating and sustaining historical trauma as well as other localized other approaches to addressing historical trauma within Native communities.

\section{Community-Based Participatory Research (CBPR) Implications}

This case study's analysis of the FGC's trauma-informed community-based participatory research and planning contributes to the body of literature by adding historical trauma into the methodology of CBPR. Most of the literature on CBPR is discussed through the first-hand experience of the academic researcher. By using a qualitative sociological approach, this study increases the voices of Portland Native 
community members within the methodological discussions of CBPR. This brought out FGC members' perspectives of the trauma-informed community-based participatory research and planning as a healing process and an intervention in the relationships between government agencies and the Portland Native community.

By framing research and planning as a social process, the analysis from this case study helps us to understand collaborative partnerships and processes in a sociological way. Most CBPR literature focuses on the barriers to collaboration that are rooted in historical trauma and mistrust between Native communities and the universities. (Brayboy and Deyhle 2000). This study highlights the ways in which collaborative partnerships for research and planning between government agencies and Native communities are especially tenuous considering the government agencies' more active role in colonization.

Historical trauma and mistrust between government agencies and Native communities is much higher than historical trauma and mistrust in the relationships between universities and Native communities. The findings suggest more extreme barriers to community-based participatory research and planning within the partnerships between government agencies and Native communities. Additionally, the use of community-based participatory research and planning within this context provides greater potential for systems change. FGC members continually suggest that the traumainformed model of community-based participatory research and planning could be adapted to address other health inequalities in Native communities as well as other communities of color. 
The findings from this study suggest a holistic way of looking at the mechanisms of CBPR by highlighting overlaps between building community capacity, Native representation, and Native-driven processes. FGC members described all three of these mechanisms as mutually reinforcing and absolutely necessary for healing the partnerships between government agencies and Native communities as well as for the engagement of Native communities. FGC members were also critical of some of the white-western ways in which community-based participatory research and planning is framed within the existing body of literature. In this way, colonized terms of community-based participatory research and planning were not always useful within collaborative setting. FGC members also highlighted the ways in which CBPR processes are really "old ways" of traditional Native American approaches to healing.

These findings contribute to our academic understanding of decolonizing the community-based participatory research and planning process. This research provides into CBPR within Native communities by highlighting the successes and barriers of the FGC's innovative trauma-informed approach to research and planning. Future research should look into how the FGC's trauma-informed research and planning process is evaluated by various stakeholders who are not members of the collaborative. Additional research is needed to evaluate the continued affects of the FGC's trauma-informed process over the long term. More broadly, research should continue to examine the implications of community-based participatory research and planning from the perspectives of the research teams.

Finally, this study makes a methodological contribution to the CBPR literature by outlining the methods of community-based participatory evaluation (see Chapter 4: 
Methods, p. 40). As a researcher, I too experienced various shifts by integrating traumainformed research and planning principles throughout the research process. This helped me to confront disinvestment into Native communities, Native exclusion, and whitewestern dominated processes within my own research design. This work had an especially significant impact on me as researcher, coming from the field of sociology where CBPR methodologies are rare. More research is needed to explore effective methods of integrating community-based participatory research principles into evaluation methodologies as well as into the field of sociology.

\section{Conclusion}

The FGC's trauma-informed process has also been demonstrated to effectively address structural inequalities, enabling ideologies, and discrimination within the partnerships between government agencies and the Portland Native community. In this way, the FGC's trauma-informed process can be seen as successful tool for working to dismantle the systemic transmission of historical trauma within the collaborative setting. This provides an upstream approach to reducing the structural inequalities that create disparities in terms of Native social determinants of health through collaborative partnerships between government agencies and Native communities.

By outlining the government's role creating and sustaining historical trauma, government agencies could also proactively work to adjust policy in order to reduce structural inequalities. This could help to alleviate some of the systemic perpetuation of historical trauma. Specifically, FGC members describe how government agencies can address their legacy of disinvestment into Native communities by investing into 
partnerships and programs within Native communities. The FGC specifically needs funding in order to continue the work in the Portland Native community. Government agencies can likewise reduce Native exclusion by creating space for Native representation within government agencies. This could be accomplished within the FGC if more government agencies agreed to join the collaborative and participate in the trauma-informed process of partnership development. Lastly, government agencies needs to let go of white-western dominated processes that create barriers to collaboration and limit the effectiveness of programing within the Native community. This could be achieved by government agencies engaging in Native-driven approaches that increase Native autonomy over research and planning. Within the FGC, government agencies could let go of bureaucratic policies that create hierarchical, top-down, one-size fits all, linear, and outcome-oriented approaches to public health.

The primary lesson from the FGC's trauma-informed model was the importance of shifting the focus of research and planning away from achieving specific set of narrow outcomes. Rather, the successes and barriers of the FGC's trauma-informed model suggest that a Native-driven research and planning process was the only viable for option addressing historical trauma and the substance-exposed pregnancies within the Portland Native community. The endemic nature of the historical trauma process within governmental agencies requires this Native representation in order to guide the evolution of this holistic healing process. In this way, the significance of Native self-determination within partnerships between government agencies and the Portland Native community cannot be understated. 
FGC members outline the ways in which Portland Native community members have been able to successfully resist the systemic transmission of historical trauma by government agencies through a healing process. This opens discussion on how government agencies can be accountable for their role in creating and sustaining historical trauma. The increase in knowledge about historical trauma and the relationships between government agencies and the Portland Native community could be useful in preventing future colonial projects that create historical trauma in Native communities. This research further sheds light on how government agencies can make systems change to stop perpetuating historical trauma. The results of this case study also supports government agencies in taking an active role in support Native protective factors, thereby contributing to Native resilience and healthier relationships between government agencies and Native communities. Finally, this case study contributes to our understanding of how Native communities can promote resistance, resilience, and healing from the systemic perpetuation of historical trauma. 


\section{References}

Brave Heart, Maria Yellow Horse. 1998. “The Historical Trauma Response Among Natives and Its Relationship with Substance Abuse: A Lakota Illustration.” Journal of Psychoactive Drugs 35(1).

Brave Heart, Maria Yellow Horse and Lemyra M. DeBruyn. 1998. "The American Indian Holocaust: Healing Historical Unresolved Grief." American Indian and Alaska Native Mental Health Research 8(2).

Brave Heart, Maria Yellow Horse, Josephine Chase, Jennifer Elkins, and Deborah B. Altschul. 2011. "Historical Trauma Among Indigenous Peoples of the Americas: Concepts, Research, and Clinical Considerations.” Journal of Psychoactive Drugs 43(4).

Brayboy, Bryan McKinley and Donna Deyhle. 2000. "Insider-Outsider: Researchers in American Indian Communities." Theory Into Practice 39(3).

Center for Disease Control and Prevention. 2009. "Tracking fetal alcohol syndrome." Atlanta, GA: Center for Disease Control and Prevention. Retrieved from: http://www.cdc.gov/ncbddd/fas/fassurv.htm

Canales, Mark K., Diane Weiner, Markos Samos, Nina S. Wampler. 2011. "MultiGenerational Perspectives on Health, Cancer, and Biomedicine: Northeastern Native American Perspectives Shaped by Mistrust." Journal of Health Care for the Poor and Underserved 22(2001).

Chino, Michelle and Lemyra DeBruyn. 2006. "Building True Capacity: Indigenous Models for Indigenous Communities." American Journal of Public Health 96(4).

Churchill, Ward. 2004. Kill the Indian, Save the Man: The Genocidal Impact of American Indian Residential Schools. San Francisco, CA: City Lights Books.

Curry-Stevens, A., Cross-Hemmer, A. \& Coalition of Communities of Color. 2011. The Native American Community in Multnomah County: An Unsettling Profile. Portland, OR: Portland State University.

Czyzewski, Karina. 2011. "Colonialism as a Broader Social Determinant of Health." The International Indigenous Policy Journal 2(1).

Evans-Campbell, Teresa. 2008. "Historical Trauma in American Indian/Native Alaska Communities: A Multilevel Framework for Exploring Impacts on Individuals, Families, and Communities." J Interpers Violence 23(3).

Fast, Elizabeth and Delphine Collin-Vézina. 2010. "Historical Trauma, Race-Based Trauma and Resilience of Indigenous Peoples: A Literature Review." First Peoples Child \& Family Review 5(1).

Gone, Joseph P. 2009. "A Community-Based Treatment for Native American Historical Trauma: Prospects for Evidence-Based Practice." Journal of Consulting and Clinical Psychology 77(4).

Grande, Sandy. 2004. Red Pedagogy: Native American Social and Political Thought. Lanham, MD: Rowman \& Littlefield.

Haynes Writer, Jeanette. 2008. "Unmasking, Exposing, and Confronting: Critical Race Theory, Tribal Critical Race Theory, and Multicultural Education. International Journal of Multicultural Education 10 (20).

Healey, Joseph. 2014. Diversity and Society: Race, Ethnicity, and Gender, $4^{\text {th }}$ Edition. 
Thousand Oaks, CA: Sage.

Holkup, Patricia A., Toni Tripp-Reimer, Emily Matt Salois, and Clarann Weinert. 2009.

"Community-based Participatory Research: An Approach to Intervention

Research With a Native American Community." ANS Adv Nurs Sci 27(3).

Israel, Barbara A., Amy J. Schulz, Edith A. Parker, and Adam B. Becker. 1998. "Review of Community-Based Research: Assessing Partnership Approaches to Improve Public Health." Annual Review of Public Health 19(1): 173-202.

Johnson, Troy, Joane Nagel, and Duane Champagne, eds. 1997. American Indian Activism. Chicago, IL: University of Illinois Press.

LaFramboise, Teresa, Dan R. Hoyt, Lisa Oliver, and Les B. Whitbeck. 2006. "Family, Community, and School Influences on Resilience Among American Indian Adolescents in the Upper Midwest." Journal of Community Psychology 34(2).

Marshall, Catherine and Gretchen B. Rossman. 2011. Designing Qualitative Research. Thousand Oaks, CA: Sage.

McKinley, Bryan and Jones Brayboy. 2005. "Toward a Tribal Critical Race Theory in Education." The Urban Review 37(5).

Mehl-Madrona, Lewis. 2000. "Psychosocial Prenatal Intervention to Reduce Alcohol, Smoking and Stress and Improve Birth Outcomes Among Minority Women." Journal of Prenatal and Perinatal Psychology and Health 14(3-4), 257-278.

Mihesuah, Devon Abbott. 2003. Indigenous American Women: Decolonization, Empowerment, Activism. Lincoln, NE: University of Nebraska Press.

Myrdal, Gunnar. 1962. An American Dilemma: The Negro Problem and Modern Democracy. New York, NY: Harper \& Brothers Publishers.

Northwest Portland Area Indian Health Bureau. 2003. Fetal Alcohol Syndrome. Portland, OR. Retrieved April 3, 2010.

(http://www.npaihb.org/health issues/fetal alcohol syndrome).

Rentner, Terry L., Lynda Dee Dixon, and Lara Lengel. 2012. "Critiquing Fetal Alcohol Syndrome Health Communication Campaigns Targeted to American Indians." Journal of Health Communication: International Perspectives 7(1).

Rosenthal, Nicolas Gabler. 2000. “'Walk Across the Bridge....nn' You'll Find Your People': Native Americans in Portland, Oregon, 194." Master's Thesis, Department of History, University of Oregon, Eugene.

Salmon, Amy. 2011. "Aboriginal Mothering, FASD prevention and the Contestations of Neoliberal Citizenship." Critical Public Health 21(2).

Sarche, Michelle C., Candace Fleming, and Paul Spicer. 2011. "The Prevention of Fetal Alcohol Spectrum Disorders in Tribal Communities." Pp. 233-254 in Child Psychology and Mental Health: American Indian and Alaska Native Children and Mental Health: Development, Context, Prevention, and Treatment, edited by P. Spicer, P. Farrell, M.C. Sarche, and H.E. Fitzgerald. California: Praeger.

Shalala, D., M. H. Trujillo, G. J. Hatz and A. J. D'Angelo. 1999. Regional Differences in Indian Health: 1998-1999. Rockville, MD: U.S. Department of Health and Human Services.

Smith, Andrea. 2007. "Soul Wound: The Legacy of Native American Schools." Amnesty International Magazine, March 26. Retrieved April 25, 2014. (http://www.amnestyusa.org/node/87342). 
Smith, Linda Tuhiwai. 1999. Decolonizing Methodologies: Research and Indigenous Peoples. London: Zed Books.

Sotero, Michelle M. 2006. “A Conceptual Model of Historical Trauma: Implications for Public Health Practice and Research.” Journal of Health Disparities Research and Practice 1(1).

Springett, Jane and Nina Wallerstein. 2008. "Issues in Participatory Evaluation.” Pp. 200223 in Community-Based Participatory Research for Health, edited by M. Minkler and N.B. Wallerstein. San Francisco, CA: Jossey-Bass.

Strickland, June C. 1999. "Conducting Focus Groups Cross-Culturally: Experiences with Pacific Northwest Indian People." Public Health Nursing 16(3).

Strickland, June C. 1999. "The Importance of Qualitative Research in Addressing Cultural Relevance: Experience from Research with Pacific Northwest Indian Women." Health Care for Women International (20).

Strickland, June C. 2006. "Challenges in Community-Based Participatory Research Implementation: Experiences in Cancer Prevention With Pacific Northwest American Indian Tribes." Cancer Control 13(3).

Substance Abuse and Mental Health Services Administration. 2014. "About Screening, Brief Intervention, and Referral to Treatment (SBIRT)". Retrieved April 25 , 2014. http://beta.samhsa.gov/sbirt/about

Thomas, Lisa Rey, Dennis M. Donovan, Robin Little Wing Sigo, and Laura Price. 2011. "Community-Based Participatory Research in Indian Country. Definitions, Theory, Rationale, Examples, and Principles." Pp. 165-187 in Child Psychology and Mental Health: American Indian and Alaska Native Children and Mental Health: Development, Context, Prevention, and Treatment edited by P. Spicer, P. Farrell, M.C. Sarche, and H.E. Fitzgerald. Santa Barbara, CA: Praeger.

Wallerstein, Nina B. and Bonnie Duran. 2006. "Using Community-Based Participatory Research to Address Health Disparities." Health Promot Pract 7(312).

Wallerstein, Nina B. and Bonnie Duran. 2008. "Theoretical, Historical, and Practice Roots of CBPR.” Pp. 26-46 in Community-Based Participatory Research for Health, edited by M. Minkler and N.B. Wallerstein. San Francisco: Jossey-Bass.

Walters, Karina L., Antony Stately, Teresa Evans-Campbell, Jane M. Simoni, Bonnie Duran, Katie Schultz, Erin C. Stanley, Chris Charles, and Deborah Guerrero. 2009. "'Indigenist' Collaborative Research Efforts in Native American Communities." Pp. 146-262 in Field Research Survival Guide edited by A.R. Stiffman. New York, NY: Oxford University Press.

Walters, Karina L. and Jane M. Simoni. 2002. "Reconceptualizing Native Women's Health: An 'Indigenist' Stress-Coping Model." American Journal of Public Health 92(4).

Walters, Karina L. and Jane M. Simoni. 2009. "Decolonizing Strategies for Mentoring American Indians and Alaskan Natives in HIV and Mental Health Research." American Journal of Public Health 99(S1). 


\section{Appendix A: Interview Guide}

Throughout this interview, please feel free to say as much as a little you like. You are welcome to share any stories and experiences that you feel are relevant. Please also let me know if any of my questions are unclear or if you think there is anything that you would like to add that I'm missing.

\section{Demographics}

The first part of the study is asking about your background information. This will be helpful for getting a sense of how your work within the FGC fits into the bigger picture of your life as well as collecting information on diversity within the FGC.

1. Can you tell me about yourself, who you are, your tribe, and where you come from?

2. Can you tell me about what your life looks like today and what keeps your busy? This could be work, family, care of others, community involvement.

\section{FGC Participation}

3. Tell me about your involvement within the Future Generations Collaborative (FGC), starting from when you first heard of the FGC?

4. How do you feel about your level of involvement in the FGC?

\section{Building Community Capacity}

5. What does building community capacity mean to you in the context of partnerships between government agencies and Native communities? Do you think it is important? If so, how?

6. To what extent do you think the FGC has built community capacity in the Portland Native community? What has the FGC's work mean to you, your family, community? Can you give examples?

7. On a personal level, what have you gained from your work with the FGC? This could be resources, goals/ambitions, insights, opportunities, financial compensation etc.

\section{Equal Partnership}

8. What does equal partnership mean to you in the context of government agencies working with Native Communities? Is it important?

9. To what extent do you think Native voices and interests are represented within the FGC's decision making?

10. What does leadership mean to you? To what extent do you think that Native people hold leadership positions within the FGC?

11. Do you feel that you personally have the opportunity to contribute to decision making within the FGC? Can you give an example?

\section{Rapport}

12. When thinking about government agencies working with the Portland Native community, what does trust mean to you? Do you think it is important? 
13. To what extent do you think the FGC has created trust with Portland Native community members? Can you give examples?

14. To what extent do you see the various partners in the FGC (this could include government agencies, Native serving organizations, the Elders and Natural Helpers) making long-term commitments to their work with the FGC?

\section{Historical Trauma}

15. What does historical trauma mean to you/to the Portland Native Community? Do you think it is important in the context of government agencies working with Native American populations?

16. Can you give any examples of how the FGC uses the concept of historical trauma in their research or planning process?

17. Do you see the FGC as working toward healing historical trauma?

\section{Cultural Appropriateness}

18. What does cultural appropriateness mean to you/community in the context of government agencies working with Native American peoples?? Why are such efforts important/not important?

19. In what ways do you see the FGC's work in the Portland Native community as culturally appropriate or not? Can you give some examples?

20. Overall, would you say that the FGC embrace Native traditional knowledge and worldviews? Can you give an example?

\section{Social and Political Impacts}

21. What are the potential impacts of the FGC's work to address substance-exposed pregnancies in the Portland Native American community?

22. What are the potential impacts of the FGC's to create collaboration between the Portland Native community and Governmental health and social services?

23. What are the potential impacts of the FGC's trauma-informed model?

24. What are the potential impacts of the FGC's work in building community capacity?

\section{Closing}

25. Is there anything else you would like to share about anything we've talked about today/tonight?

26. Do you have any questions for me?

27. Can I get your email address? 


\section{Appendix B: Codebook Revisions and Themes}

The table presented below demonstrates how the coding schema from this case study was revised over the course of data analysis. Additionally, the table presented documents how codes from the data were built into themes in order to address this case study's research questions. For a more detailed description of the data analysis process see Chapter 4: Methods.

Table 2: Codebook Revisions and Themes

\begin{tabular}{|c|c|c|}
\hline Initial Code Categories & Revised Codes & Themes \\
\hline $\begin{array}{l}\text { Historical Trauma }+ \\
\text { The Shift }+ \\
\text { Healing }\end{array}$ & $\begin{array}{l}\text { Intergenerational } \\
\text { Transmission }+ \\
\text { Systemic Transmission }+ \\
\text { Native Resistance and } \\
\text { Resilience }+ \\
\text { County as Authentic } \\
\text { Allies }+ \\
\text { Healing from Historical } \\
\text { Trauma }\end{array}$ & $\begin{array}{l}\text { The Historical Trauma } \\
\text { Process + } \\
\text { The Shift to a Trauma- } \\
\text { Informed Model + } \\
\text { The Trauma-Informed } \\
\text { Healing Process }\end{array}$ \\
\hline $\begin{array}{l}\text { Status Quo: Unequal } \\
\text { Relationships }\end{array}$ & $\begin{array}{l}\text { Government } \\
\text { Disinvestment }+ \\
\text { Native Exclusion }+ \\
\text { White-Western } \\
\text { Dominated Processes } \\
\end{array}$ & $\begin{array}{l}\text { The Systemic } \\
\text { Transmission of } \\
\text { Historical Trauma }\end{array}$ \\
\hline Trauma-Informed Process & $\begin{array}{l}\text { Building Community } \\
\text { Capacity }+ \\
\text { Equal Partnership }+ \\
\text { Cultural Appropriateness }\end{array}$ & $\begin{array}{l}\text { Investment into Native } \\
\text { Communities }+ \\
\text { Native Representation }+ \\
\text { Native-Driven Processes }\end{array}$ \\
\hline Impacts of FGC's Work & $\begin{array}{l}\text { Partnership Impacts }+ \\
\text { Native Health Impacts }\end{array}$ & $\begin{array}{l}\text { Impacts on the } \\
\text { Relationships between } \\
\text { Government Agencies } \\
\text { and Native Communities } \\
+ \\
\text { Impacts on Native } \\
\text { Health, Social, Political, } \\
\text { and Economic Outcomes }\end{array}$ \\
\hline
\end{tabular}

